\section{Check for updates}

Cite this: Org. Chem. Front., 2020, 7 3815

Received 28th June 2020,

Accepted 19th September 2020

DOI: $10.1039 / \mathrm{d} 0$ qo00763c

rsc.li/frontiers-organic

\title{
Construction of all-carbon quaternary stereocenters by catalytic asymmetric conjugate addition to cyclic enones in natural product synthesis
}

\begin{abstract}
Zhuo Wang (D)
Asymmetric catalysis for chiral compound synthesis is a rapidly growing field in modern organic chemistry and provides enantioselective materials to meet the demands of various fields. However, the construction of all-carbon quaternary stereocenters poses a distinct challenge in organic synthesis. The development of catalytic asymmetric conjugate additions that require only a catalytic amount of a transition metal with a chiral ligand or organocatalyst has provided an efficient approach to the preparative-scale synthesis of enantioselective and/or diastereoselective conjugate adducts. Such reactions have been used in various synthetic applications such as natural product synthesis and reports of the use of this approach are becoming increasingly common in the literature. In particular, tandem copper-catalyzed asymmetric conjugate addition/enolate trapping by a carbon electrophile enables diastereoselective synthesis of $\alpha, \beta$-substituted ketones with contiguous stereogenic centers, which is still an intricate task in organic synthesis. In this review, the use of asymmetric conjugate addition in natural product synthesis is described and discussed in depth.
\end{abstract}

\section{Introduction}

Southern University of Science and Technology, School of Medicine, Shenzhen, 518055, People's Republic of China. E-mail: wangz3@sustech.edu.cn

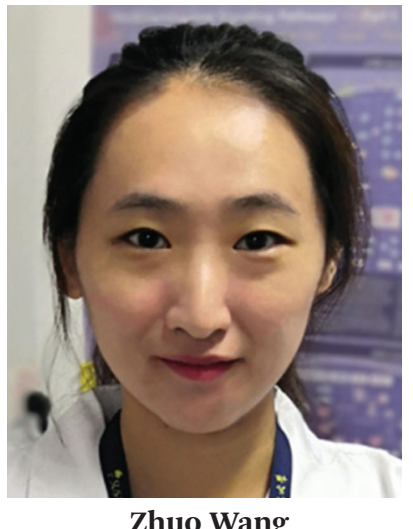

Zhuo Wang
Zhuo Wang received her BSc from Wuhan University (2009) and PhD from The Hong Kong Polytechnic University (2014) working under the direction of Dr Tao Ye. After working as a research associate in the same university for one year, she joined the lab of Prof. Hongmin Zhang at the Southern University of Science and Technology (aka. SUSTech) from 2016 to 2018 for post-doctoral research, followed by working as a senior research fellow. In late 2019, she accepted her professional appointment as a Research Assistant Professor from the School of Medicine of SUSTech. Her research interests include studying synthesis and biological evaluation of active natural products and small molecules, exploring pathogenesis of diseases, and investigating novel molecular-targeted therapeutic strategies.
Asymmetric catalysis for chiral compound synthesis is a rapidly growing field in modern organic chemistry and provides enantioselective materials that meet the demands of various fields. However, the construction of all-carbon quaternary stereocenters still poses a distinct challenge in organic synthesis and the development of methods for constructing such centers is imperative. ${ }^{1-11}$ Innovative chiral catalysts have enabled the efficient construction of all-carbon quaternary centers by various asymmetric reactions such as dearomative cyclization, ${ }^{12,13}$ polyene cyclization, $^{14,15}$ arylation, ${ }^{16}$ allylation, ${ }^{17-19}$ and Michael addition. ${ }^{20}$ Catalytic asymmetric conjugate addition can be used to synthesize ketones containing all-carbon quaternary centers from enones and is an indispensable method for accessing enantiopure and/or enantioenriched materials for various synthetic purposes.

The recent success of catalytic asymmetric conjugate addition has attracted much attention from the synthetic community in terms of the development of methods for natural product synthesis. The advantages of this class of reactions, which include the use of readily accessible transition metals and chiral ligands in catalytic amounts, the ability to produce highly enantioselective and/or diastereoselective ketones containing all-carbon quaternary stereocenters, and applicability to preparative-scale reactions, collectively make catalytic asym- 
metric conjugate addition appealing for natural product synthesis. Alkylation and/or acylation at the $\alpha$-position through enolate trapping after copper-catalyzed asymmetric conjugate addition (i.e., a tandem reaction) provides diastereoselective ketones with contiguous stereocenters at the $\alpha$ - and $\beta$-positions. This approach provides access to structures with congested arrays of stereocenters. Organocatalytic asymmetric conjugate addition can establish an all-carbon quaternary stereocenter without the use of a transition-metal catalyst to generate an organometallic nucleophile. Much effort has been devoted to the development of methods for achieving catalytic asymmetric conjugate addition and this has become a facile approach to the construction of a wide range of important enantioenriched scaffolds for use in organic chemistry, medicinal chemistry, and the pharmaceutical industry. Many reviews of catalytic asymmetric conjugate addition and its applications have been published. ${ }^{21-26}$ The upsurge in the use of this class of reactions has enabled the synthesis of many structurally elusive natural products (Fig. 1). These important results have motivated us to provide a timely and focused review of catalytic asymmetric conjugate additions in natural product synthesis.

In this review, we begin with an introduction to method development and representative examples of natural product syntheses that make use of catalytic asymmetric conjugate

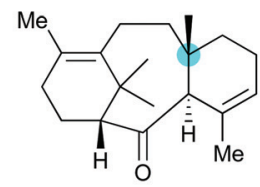

(+)-Taxadienone (1)

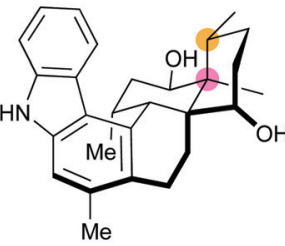

Aflavazole (6)

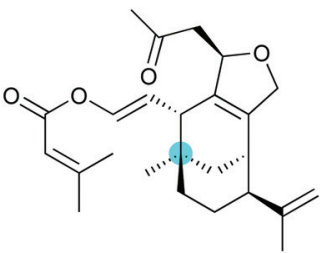

(-)-Neovibsanin G (2)

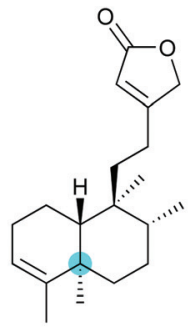

(-)-Solidagolactone (3)

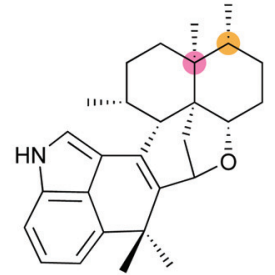

Epoxyeujindole A (4)

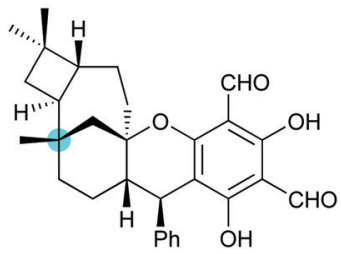

(+)-Psiguadial B (5)

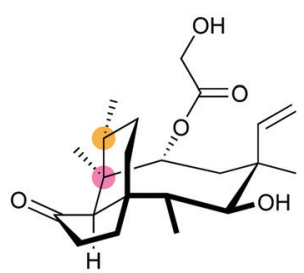

(+)-Pleuromutilin (7)

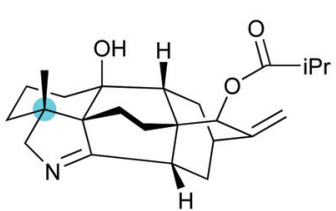

(-)-Arcutinine (8)

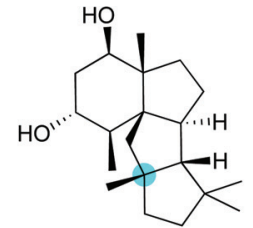

Conidiogenol (9)

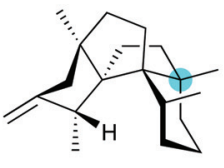

(+)-Waihoensene (10)

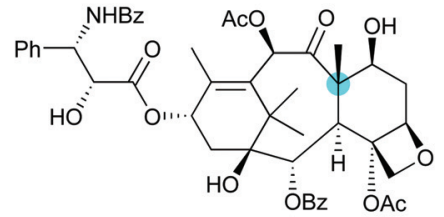

Taxol (11)

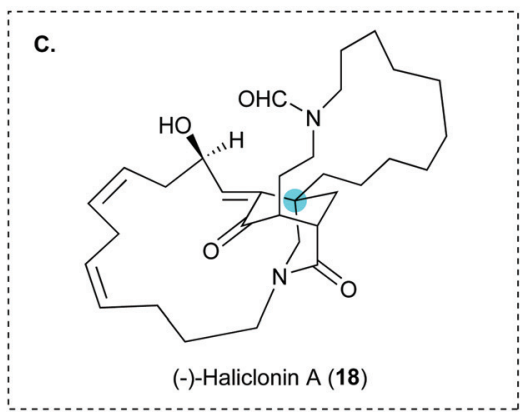

B.

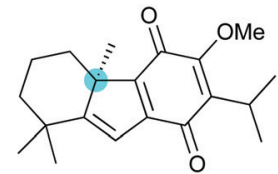

(+)-Taiwaniaquinone $\mathrm{H}$ (12)<smiles>Cc1cc2c(cc1O)C1(O)CCC2(C)CC1</smiles>

Enokipodin A (13)

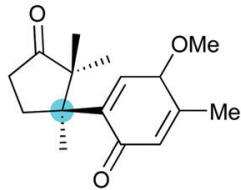

Enokipodin B (14)

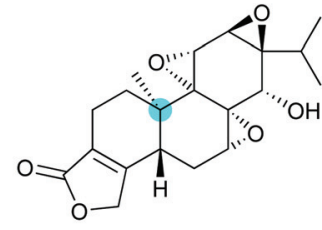

(+)-Triptolide (15)<smiles>CCC1=C2CC=C[C@@H]1CCc1c2ccc(C(C)C)c1O</smiles>

(-)-Triptophenolide (16)

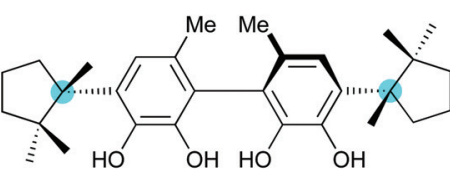

Mastigophorene A (17)

Fig. 1 Some natural product syntheses that make use of catalytic asymmetric conjugate addition (CACA) to create all-carbon quaternary stereocenters. (A) Quaternary carbon(s) created directly by copper-catalyzed asymmetric conjugate addition or indirectly by subsequent enolate trapping by carbon electrophile. (B) Quaternary carbon(s) created by palladium-catalyzed asymmetric conjugate addition. (C) Quaternary carbon(s) created by organocatalytic asymmetric conjugate addition. [Quaternary stereocenter(s) created by CACA are highlighted in cyan; stereocenter(s) created by CACA are highlighted in orange; and quaternary stereocenter(s) formed by enolate trapping immediately after CACA are highlighted in pink.] 
addition and were reported before 2016. We discuss natural product syntheses reported in 2016-2020 (inclusive) that feature this important synthetic method, with an emphasis on the key factors and transformations that led to their success. We classify the natural product syntheses according to the catalyst used in the asymmetric conjugate addition to build the all-carbon quaternary stereocenter, namely copper catalysts, palladium catalysts, and organocatalysts. Finally, we discuss potential developments and envisage future opportunities for the use of catalytic asymmetric conjugate additions.

\section{Construction of all-carbon quaternary stereocenters by copper- catalyzed asymmetric conjugate addition}

The pioneering discovery of copper-catalyzed asymmetric conjugate addition was made independently by Feringa $^{27}$ and Alexakis. ${ }^{28}$ They used a copper catalyst and a chiral phosphine ligand to facilitate asymmetric conjugate addition of an enone to give an enantioenriched ketone (Scheme 1). Chirality was

\section{A. Feringa 1997}
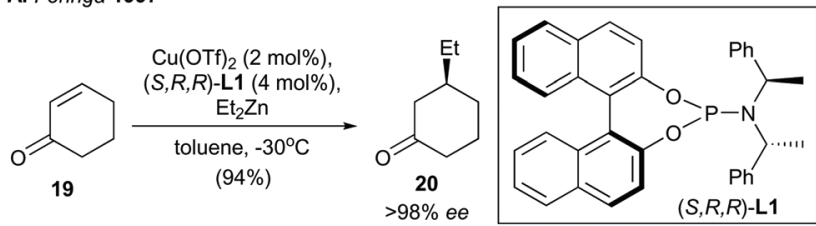

B. Alexakis 2002
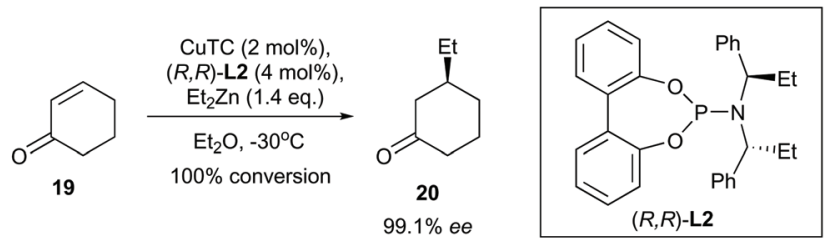

C. Proposed mechanism by Feringa

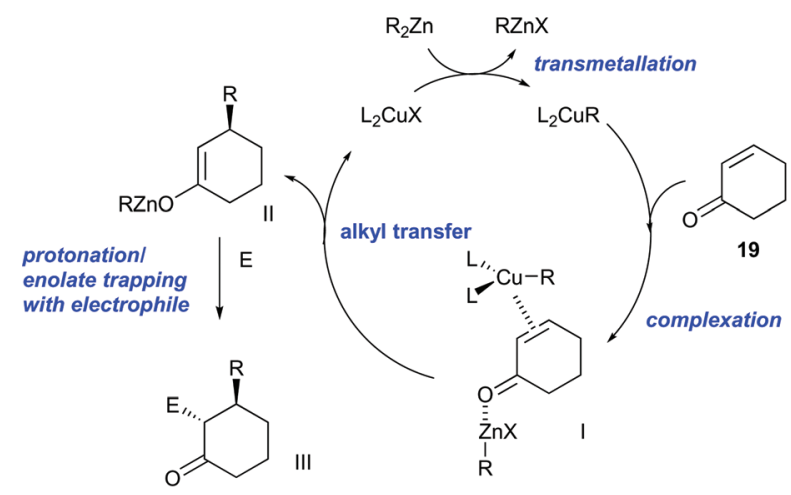

Scheme 1 Pioneering reports of copper-catalyzed asymmetric conjugate addition by (A) Feringa $(1997)^{27}$ and (B) Alexakis (2002). ${ }^{28}$ (C) Mechanism proposed by Feringa. ${ }^{29}$ introduced by using a chiral phosphoramidite ligand [e.g., $(S$, $R, R)$-L1 and $(R, R)-\mathbf{L} 2]$. The copper-catalyzed asymmetric conjugate addition of cyclohexenone (19) with diethylzinc produced the enantioenriched ketone $\mathbf{2 0}$ with a high ee value of $98 \%$ to 99.1\% (Scheme 1A and B). Feringa and co-workers ${ }^{29}$ proposed that the catalytic mechanism involves alkyl transfer from dialkylzinc (i.e., $\quad \mathrm{R}_{2} \mathrm{Zn}$ ) to a copper(I)-phosphoramidite complex, which generates a copper(I)-alkyl complex (i.e., $\mathrm{L}_{2} \mathrm{CuR}$ ) (Scheme 1C). Complexation of an alkylzinc halide (i.e., $\mathrm{RZnX}$ ) to the enone carbonyl of 19 and coordination of a copper-alkyl complex (i.e., $\mathrm{L}_{2} \mathrm{CuR}$ ) to the enone olefin of 19 results in the formation of $\pi$-complex $\mathbf{I}$. The authors reasoned that high levels of stereocontrol can result from bimetallic complex formation, which fixes the enone conformation. Next, alkyl transfer from the $\pi$-coordinated copper complex to the enone generates zinc enolate II. Finally, either protonation or trapping of the zinc enolate by an electrophile (E) produces III.

Since the report of copper-catalyzed asymmetric conjugate addition to set the enone $\beta$-stereocenter, the construction of all-carbon quaternary stereocenters has become possible and has been widely used in natural product synthesis. Representative syntheses of natural products reported before 2016 are shown in Scheme 2.

In 2011, the preparation of (+)-taxadienone (1) was reported by Baran and co-workers. The synthesis featured asymmetric conjugate addition and the enantioenriched TMS-enolate 22 was prepared in $89 \%$ yield with $93 \% \mathrm{ee}^{30}$ (Scheme 2A). In 2012, Williams and co-workers synthesized (-)-neovibsanin $\mathrm{G}(4)$ by using a $\mathrm{Cu}(\mathrm{OTf})_{2}$-chiral nitrogen-heterocyclic carbene (NHC) ligand (L4) to catalyze a conjugate addition. Use of a Grignard reagent (i.e., 26) as the alkylating agent afforded the intermediate cyclohexanone 27 in $70 \%$ yield with $91 \% \mathrm{ee}^{31}$ (Scheme 2B). Synthesis of (-)-solidagolactone (5), which was reported by Overman and co-workers in 2015, involved tandem nickel-catalyzed regioselective hydroalumination ${ }^{32,33} /$ asym- $^{2}$ metric copper-catalyzed conjugate addition $^{34}$ of enone 25 under the effect of a Ag-NHC complex $(\mathrm{Ag}-\mathbf{L} \mathbf{- 5})^{35}$ to give adduct 30 in $89 \%$ yield with $84 \%$ ee $^{36}$ (Scheme 2C). In the same year, epoxyeujindole A (6) was synthesized by Li and co-workers by asymmetric conjugate addition under the Alexakis protocol, ${ }^{37}$ followed by enolate trapping with iodide 33 to give cyclohexanone 34 in 65\% yield with a $3.8: 1 \mathrm{dr}^{38}$ (Scheme 2D).

\section{1. (+)-Psiguadial B (Reisman 2016) $)^{39}$}

(+)-Psiguadial B (5) is a diformyl phloroglucinol-containing meroterpenoid. It was isolated from the leaves of Psidium guajava by Ye and co-workers in 2010. ${ }^{40}$ This compound shows potent antiproliferative activity against human hepatoma cells (HepG2 IC $\left._{50}=46 \mathrm{nM}\right) .{ }^{40}$ In 2016, Reisman and co-workers reported the first enantioselective synthesis of $\mathbf{5}$ (Scheme 3) ${ }^{39}$ Cyclohexenone $(+)-36$, which was prepared from diazoketone 45 in five steps (Scheme 3, bottom section), was subjected to copper-catalyzed asymmetric conjugate addition via the Alexakis protocol [i.e., with $\mathrm{CuTC} /(R, S, S)-\mathbf{L} \mathbf{1}$ as the catalyst ${ }^{41}$ to afford adduct 37 in 94\% yield with a 19:1 dr. Aldol condensation of chiral adduct 37 with benzaldehyde 38 and sub- 


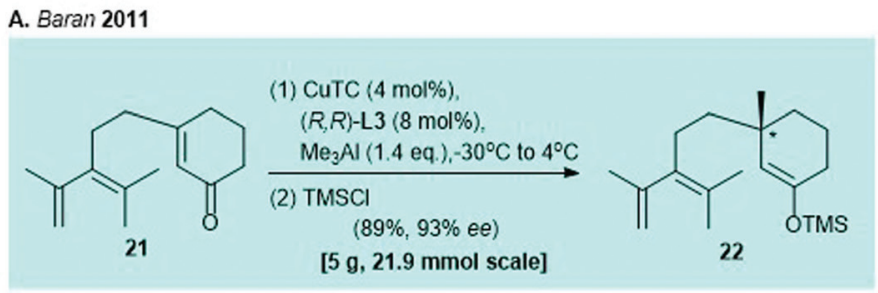

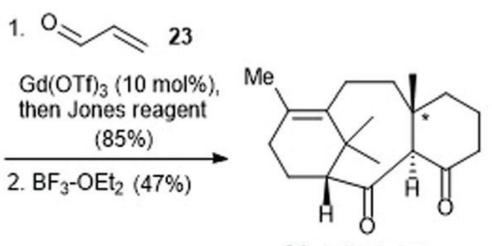

24 (>99:1 d.r.)

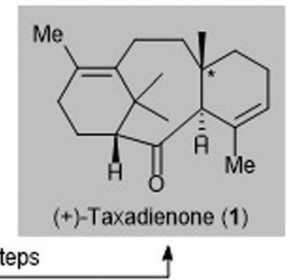

\section{B. Willams 2012}
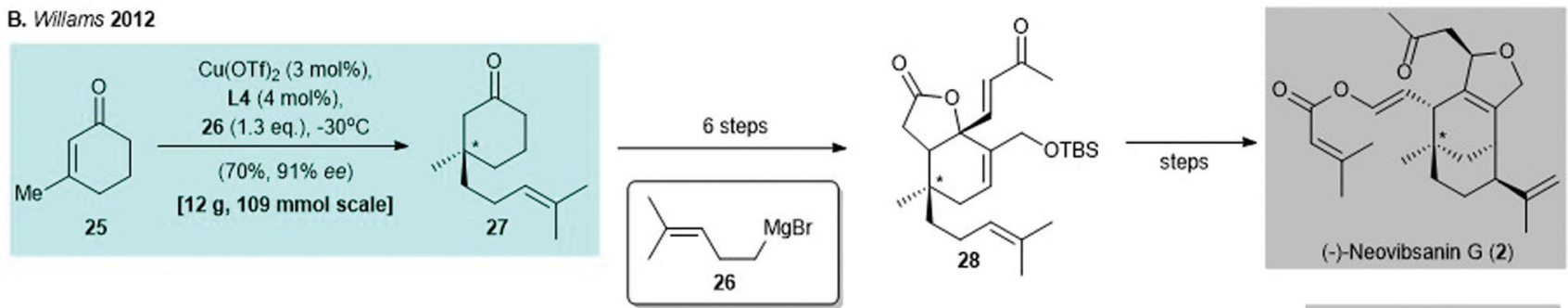

\section{Overman 2015}
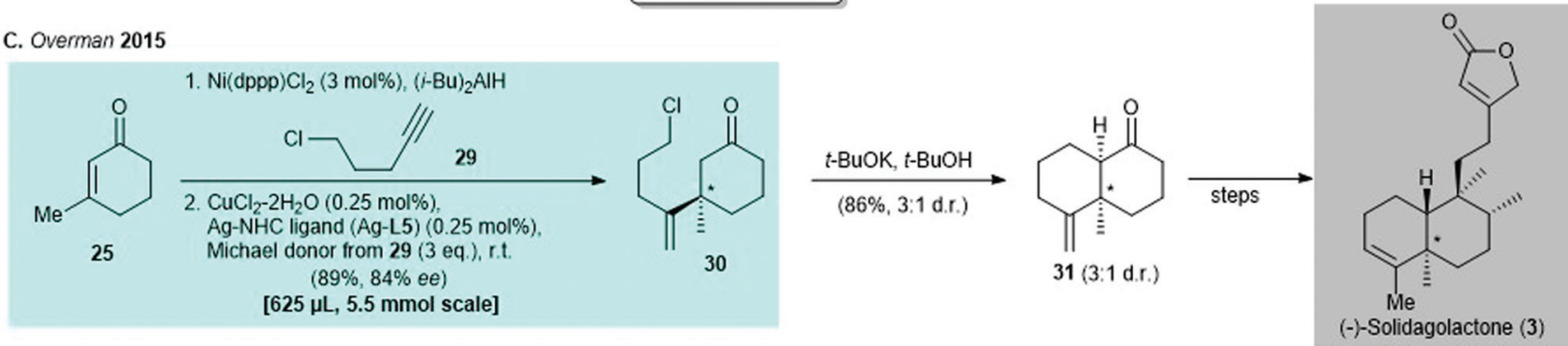

\section{Douress}

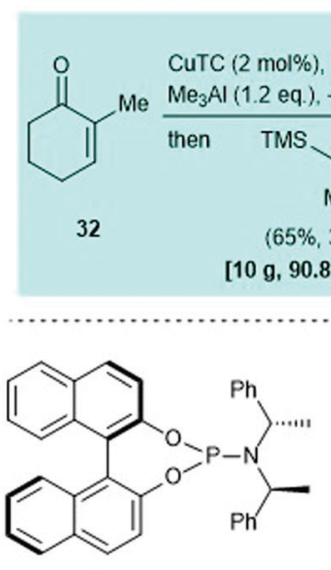

(R,S,S)-L1
(65\%, 3.8:1 d.r.)

$\mathrm{g}, 90.8 \mathrm{mmol}$ scale]

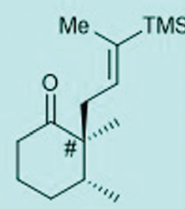

34

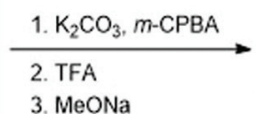

( $53 \%, 3$ steps)
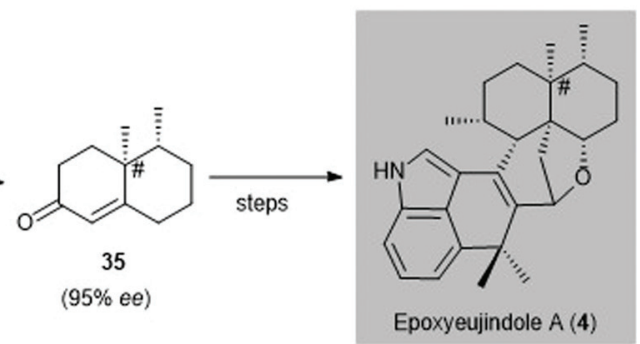<smiles>Cc1cc(C)c(OP(c2ccccc2)N(C(C)c2ccccc2)C(C)c2ccccc2)c(C)c1</smiles>

$(R, R)-\mathrm{L} 3$<smiles>Cc1cc([N+](=O)[O-])c([N+](=O)[O-])c([N+](=O)[O-])c1</smiles>

L4

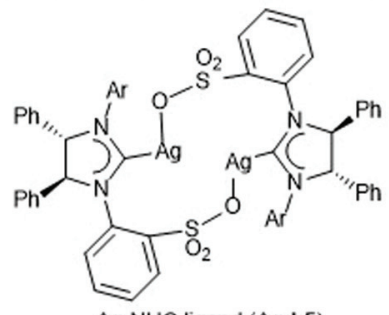

Ag-NHC ligand (Ag-L5)

( $\mathrm{Ar}=2,4,6-(\mathrm{i}-\mathrm{Pr})_{3} \mathrm{C}_{6} \mathrm{H}_{2}$ )

Scheme 2 Selected examples of natural product syntheses reported before 2016 that use copper-catalyzed asymmetric conjugate addition. [Allcarbon quaternary stereocenter(s) created by (i) copper-catalyzed asymmetric conjugate addition are denoted by *; (ii) copper-catalyzed asymmetric conjugate addition/enolate trapping is denoted by \#]. (A) Asymmetric conjugate addition of 21 and immediate trapping of enolate with trimethylsilane chloride (TMSCl) gives TMS-enolate 22 in the synthesis of (+)-taxadienone (1). (B) Synthesis of (-)-neovibsanin G (2) makes use of chiral NHC ligand L4 in catalytic conjugate addition for the preparation of enantioenriched cyclohexanone $27 .^{31}$ (C) Tandem nickel-catalyzed regioselective hydroalumination ${ }^{35}$ /asymmetric copper-catalyzed conjugate addition produces 30 in the presence of $\mathrm{Ag}-\mathrm{NHC}$ complex (Ag- $\left.\mathbf{L 5}\right)^{47}$ as a catalyst in synthesis of (-)-solidagolactone (3). ${ }^{36}$ (D) Tandem asymmetric conjugate addition/ $\alpha$-alkylation produces cyclohexanone 34 , which is a precursor in the preparation of epoxyeujindole $A(4) .^{38}$

sequent 1,2-addition with vinyllithium (39) gave alcohol 40 in $81 \%$ yield with a $2: 1 \mathrm{dr} ; 40$ was subjected to HG-II catalystpromoted ring-closing metathesis (product not shown, 93\% yield). Subsequent catalytic hydrogenation with Crabtree's catalyst produced $\mathbf{4 1}$ in $90 \%$ yield. Copper-catalyzed intramolecular $O$-arylation ${ }^{42}$ of $\mathbf{4 1}$ afforded 42 in 75\% yield; 42 was 


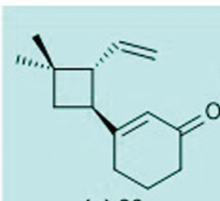
$(+)-36$

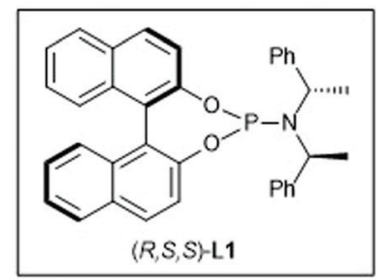
$\mathrm{Me}_{3} \mathrm{Al}\left(2 \mathrm{eq}\right.$ ),$-35^{\circ} \mathrm{C}$ (94\%, 19:1 d.r.) [0.75 g, $3.67 \mathrm{mmol}$ scale]
(15 mol\%), L1 (30 mol\%),

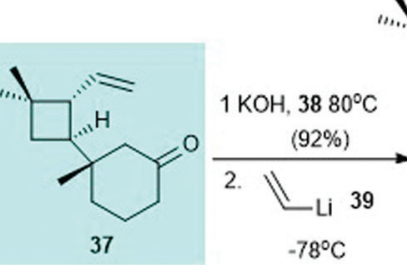

$(80 \%, 2: 1$ d.r. $)$ ( $54 \%$, single diastereomer)

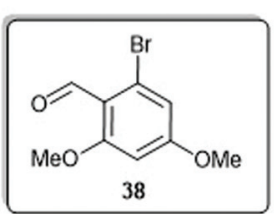<smiles>C=C[C@H]1[C@@H](C)C[C@]1(C)C1(C)CC/C(=C\c2c(Br)cc(OC)cc2OC)[C@](O)(C=C)C1</smiles>

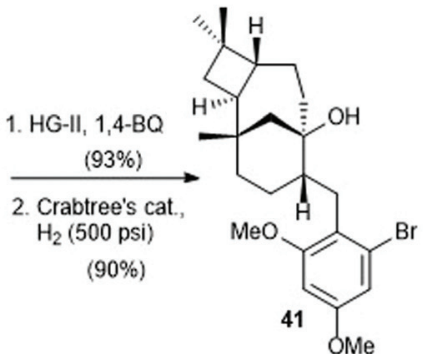
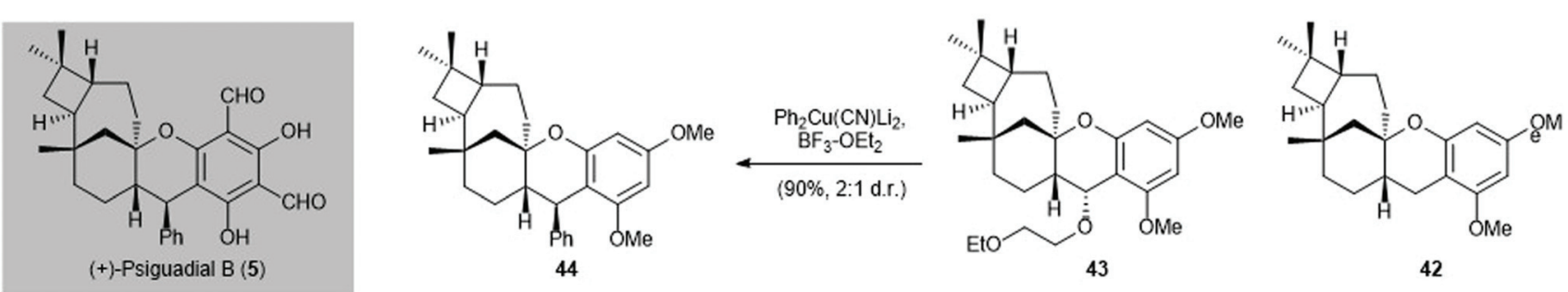

(t)-Psiguadial $B(5)$

Eto $\Upsilon_{43}^{0}$

42

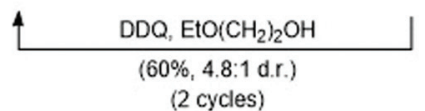

2. $\mathrm{MeOCHCl}_{2}, \mathrm{TiCl}_{4}(50 \%)$

(2 cycles)

The preparation of cyclohexenone fragment (+)-36

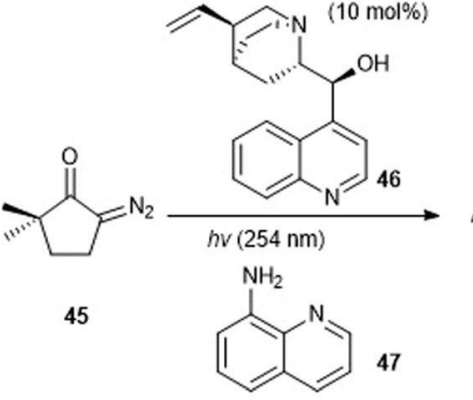

$(62 \%, 79 \% e e)$

Tandem Wolff rearrangement catalytic asymmetric ketene addition

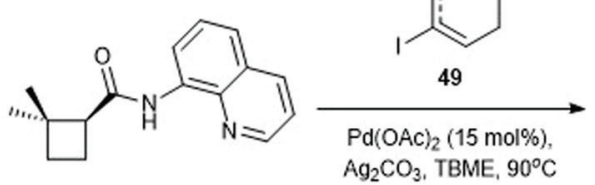

(72\%)

Palladium-catalyzed directed $C\left(\mathbf{s p}^{3}\right)-\mathrm{H}$ alkenylation

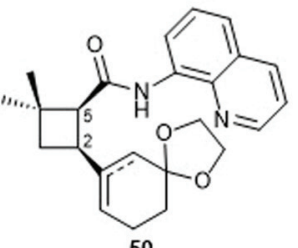

50

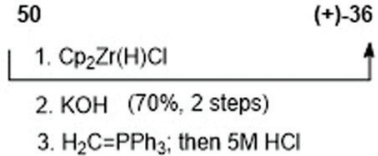

(88\%)

Scheme 3 Enantioselective synthesis of (+)-psiguadial B (5) (Reisman, 2016). ${ }^{39}$ Bottom section: preparation of cyclohexanone fragment (+)-36.

treated with $\mathrm{DDQ} /$ ethoxyethanol ${ }^{43}$ to effect benzylic oxidation to give 43 in $60 \%$ yield with a $4.8: 1 \mathrm{dr}$. Treatment of 43 with $\mathrm{Ph}_{2} \mathrm{Cu}(\mathrm{CN}) \mathrm{Li}_{2}{ }^{44,45}$ and $\mathrm{BF}_{3}-\mathrm{OEt}_{2}$ afforded 44 in $90 \%$ yield with a $2: 1 \mathrm{dr} ; \mathbf{4 4}$ was subjected to demethylation ( $62 \%$ yield, single diastereomer) and simultaneous formylation via the Rieche procedure $^{46}$ to give (+)-psiguadial B (5) in 50\% yield.

The synthesis of cyclohexenone ( + )-36 began with a tandem Wolff-rearrangement ${ }^{48} /$ asymmetric ketene addition ${ }^{49,50}$ of $\mathbf{4 5}$ with aniline $\mathbf{4 7}$, and (+)-cinchonine (46) as the catalyst, to afford enantioenriched cyclobutane $\mathbf{4 8}$ in $62 \%$ yield with $79 \%$ ee (Scheme 3, bottom section). Enantiomerically pure 48 can be obtained via single recrystallization by layer diffusion. Palladium-catalyzed cross-coupling of freshly prepared $\mathbf{4 8}$ with iodide 49 afforded $\mathbf{5 0}$ in $72 \%$ yield. Reduction of the amide group in $\mathbf{5 0}$ by $\mathrm{Cp}_{2} \mathrm{Zr}(\mathrm{H}) \mathrm{Cl}$ afforded a cis-aldehyde intermediate (not shown), which was subjected to C-5 epimerization with $\mathrm{KOH} /$ methanol, followed immediately by Wittig olefination to produce the desired enone (+)-36 in $88 \%$ yield.

\subsection{Aflavazole (Li 2016) ${ }^{51}$}

Aflavazole (6) is an indole diterpenoid. It is a metabolite of the sclerotia of Aspergillus flavus and was identified by Dowd and co-workers in $1990 .^{52}$ The first total synthesis of 6 was reported by Li and co-workers in 2016 (Scheme 4). ${ }^{51}$ Asymmetric conjugate addition of 2-methylcyclohexenone (32), catalyzed by CuTC/(R,S,S)-L1 ${ }^{38}$ enolate synthesis with methyllithium and 

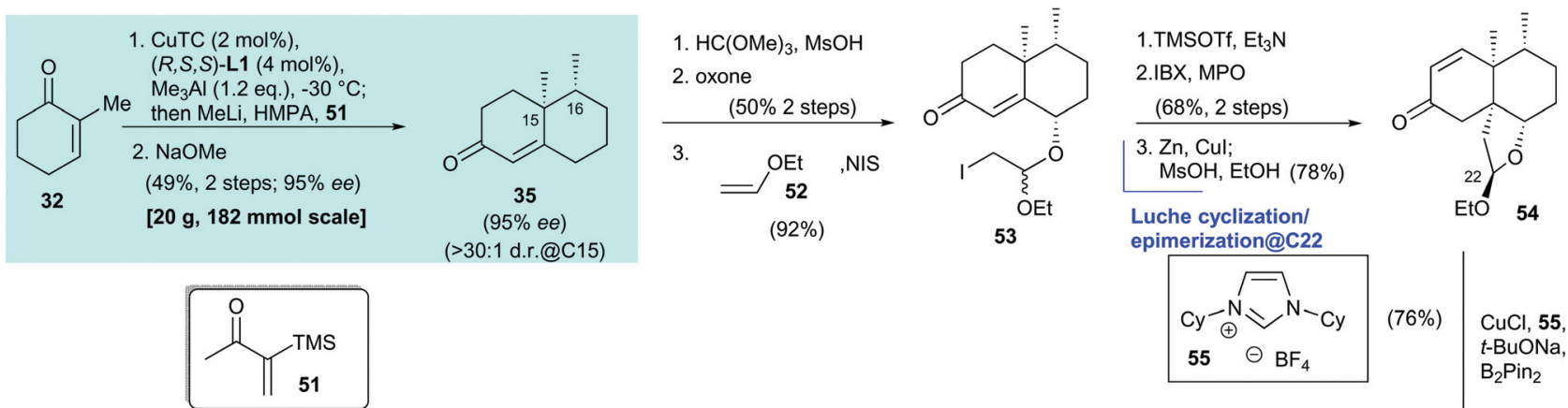

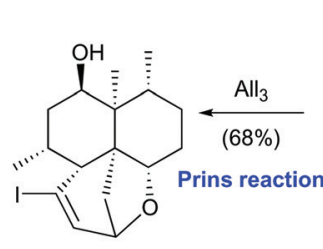

61

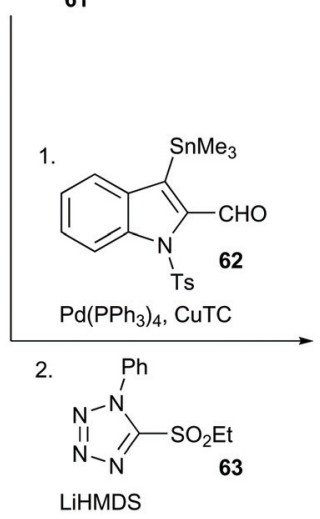

(67\%, 2 steps)

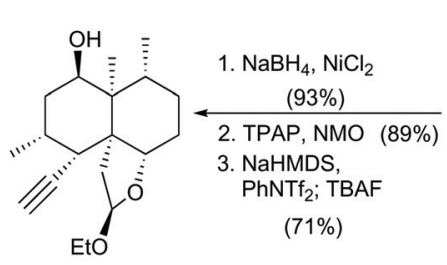

60

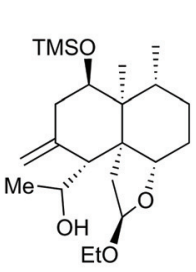

59
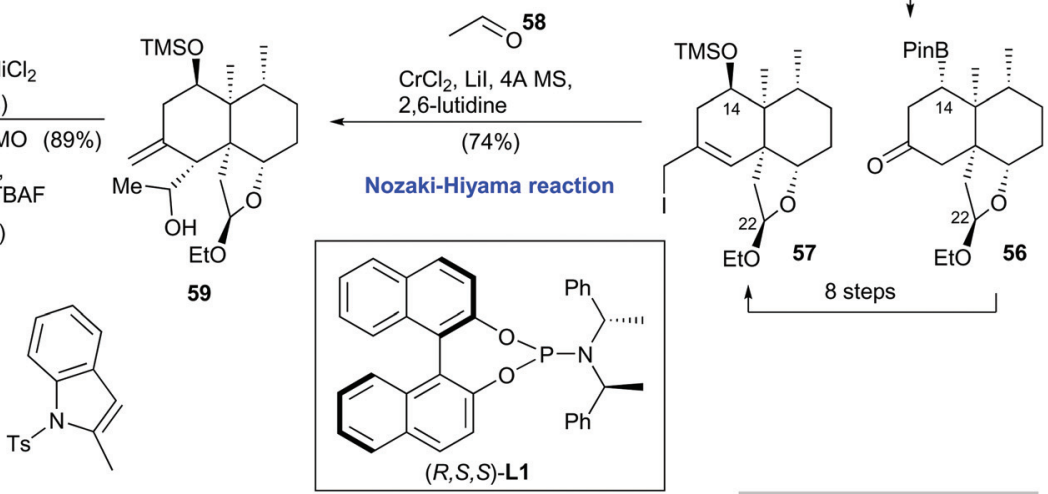

$(76 \%)$

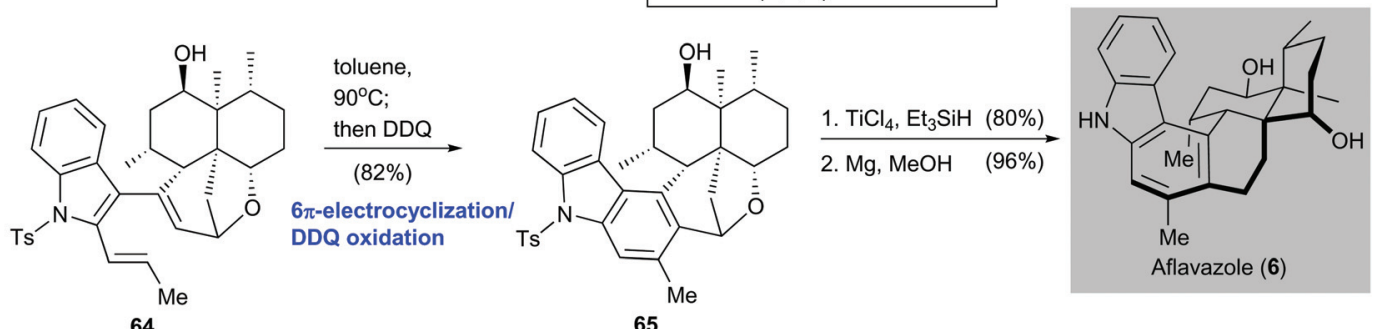

Scheme 4 Enantioselective synthesis of aflavazole (6) (Li, 2016). ${ }^{51}$

HMPA, and subsequent attack by the Stork-Ganem reagent $51^{53}$ gave an $\alpha$-silyl ketone (not shown). Exposure of the freshly prepared $\alpha$-silyl ketone to NaOMe produced 35 in $49 \%$ yield over two steps with $95 \%$ ee.

A three-step synthesis from enone 35 gave iodide 53, which was subjected to silyl enol ether formation and IBX oxdation ${ }^{54}$ to give a bis-enone (not shown, 68\% yield over two steps). The enone underwent Luche cyclization and epimerization at C-22 ${ }^{38}$ to afford enone $\mathbf{5 4}$ in $\mathbf{7 8 \%}$ yield over two steps. Boron conjugate addition $^{55,56}$ of enone 54 gave boronate 56 in 76\% yield; 56 was converted to iodide $\mathbf{5 7}$ with the desired stereochemistry at C-14 through an eight-step transformation. Freshly prepared iodide $\mathbf{5 7}$ was subjected to a Nozaki-Hiyama reaction with acetaldehyde 58 to give alcohol 59 in $74 \%$ yield as a single diastereomer. A three-step transformation from alcohol 59, namely facial selective reduction of the exo-olefin (product not shown, 93\% yield), secondary alcohol oxidation (product not shown, 89\% yield), and triflation/TBAF-mediated elimination $^{57}$ and desilylation, gave alkyne 60 in $71 \%$ yield. Treatment of 60 with $\mathrm{AlI}_{3}$ facilitated a Prins cyclization and provided the cyclization product $\mathbf{6 1}$ in $68 \%$ yield. Stille-Migita coupling of vinyl iodide 61 with tin compound 62 and subsequent Julia-Kocienski olefination afforded triene 64 in $67 \%$ yield over two steps. Heating of triene $\mathbf{6 4}$ resulted in $6 \pi$-electrocyclization, and subsequent DDQ oxidation ${ }^{58}$ gave arene 65 in $82 \%$ yield. Reductive cleavage of the benzyl ether in 65 and then desulfonation with magnesium afforded aflavazole (6) in $96 \%$ yield. The enantioselective synthesis of 14-hydroxyaflavinine (not shown) was also reported in the same publication, but is not discussed here.

\section{3. (+)-Pleuromutilin (Herzon 2017) $)^{59}$}

The enantioselective synthesis of (+)-pleuromutilin (7) was disclosed by Herzon and co-workers in $2017 ;^{59,60} 7$ is a tricyclic diterpene fungal metabolite (Scheme 5). Biologically, 7 shows inhibitory activities against the growth of Gram-positive pathogens. ${ }^{61,62}$ Because of its unique mode of action, i.e., binding to the highly conserved peptidyl transferase center of the bacterial ribosome, the development of resistance to pleuromutilin has been delayed. The synthesis of $(+)$-pleuromutilin (7) and its derivatives has therefore attracted wide interest in the context of the development of new antibiotics. ${ }^{63-66}$ 

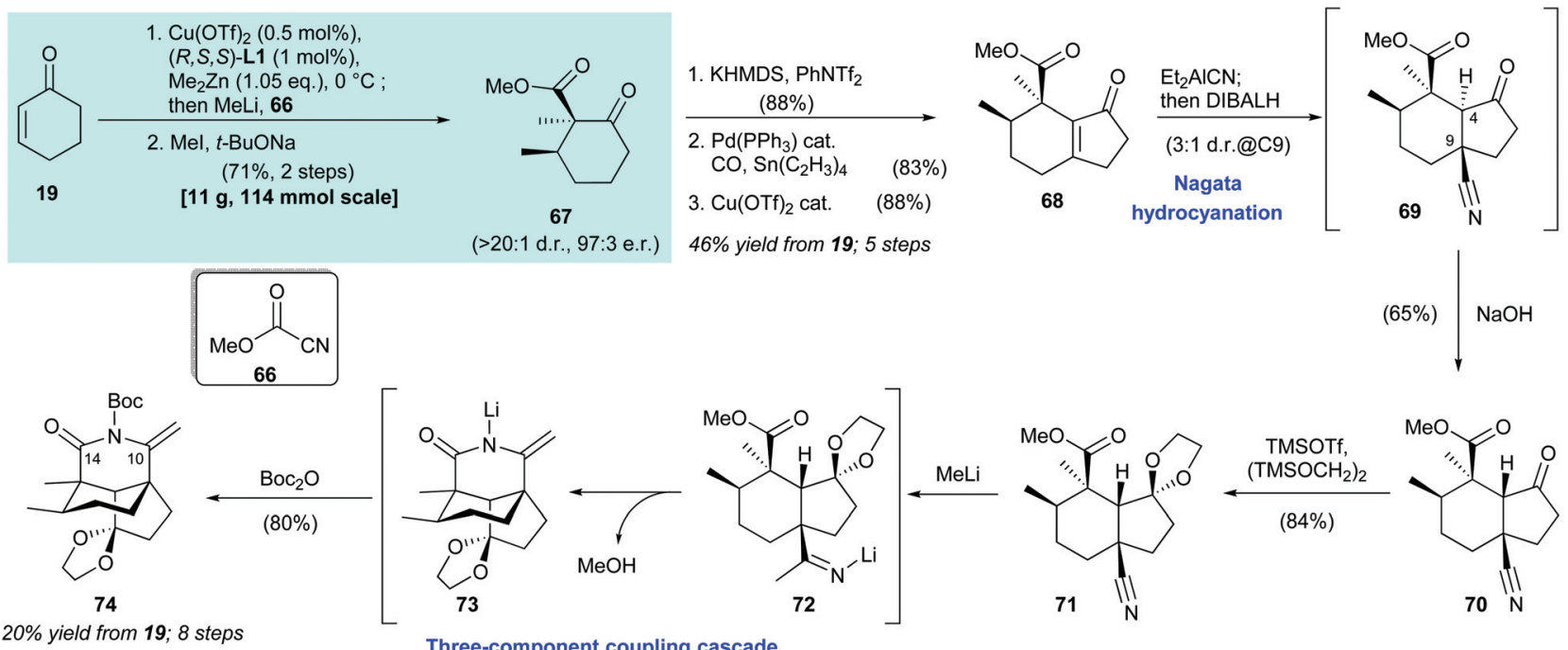

Three-component coupling cascade
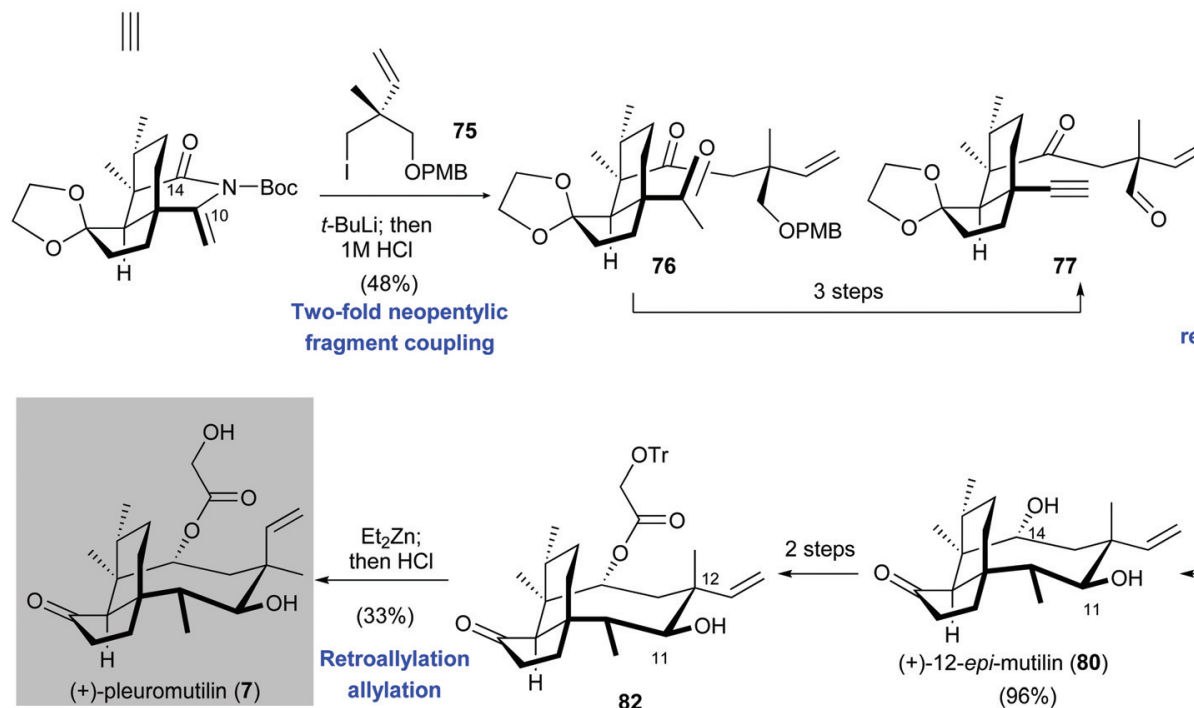

82

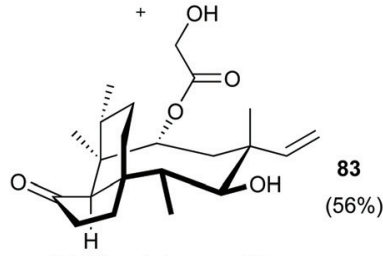

(+)-12-epi-pleuromutillin
$(56 \%)$

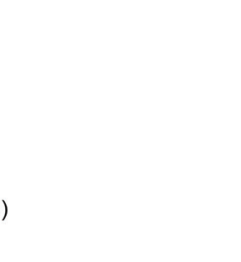

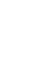

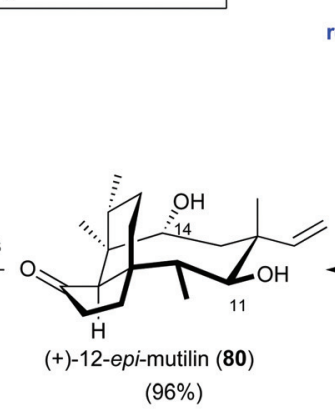

$(96 \%)$

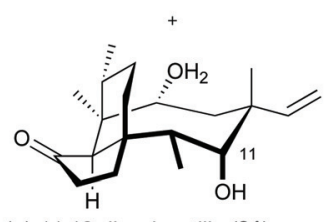

(+)-11,12-di-epi-mutilin (81)

$(81 \%)$
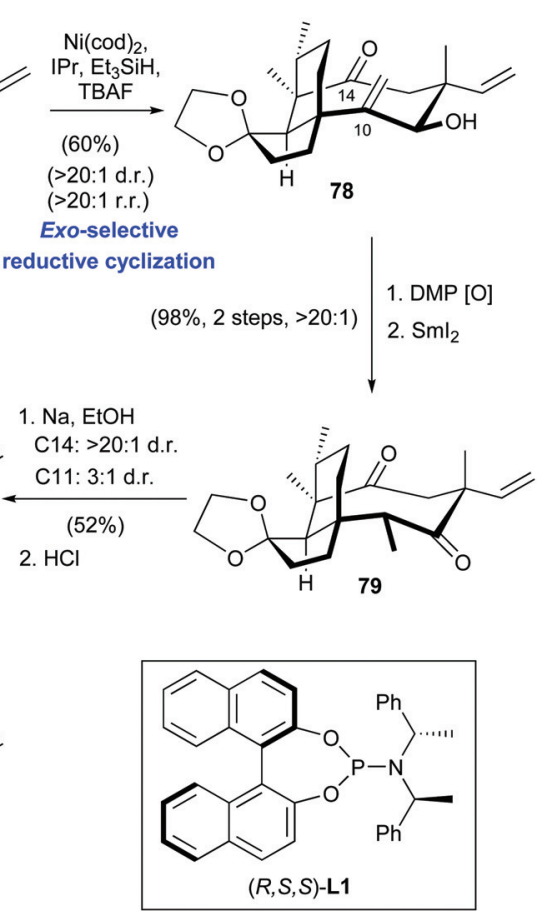

Scheme 5 Enantioselective synthesis of (+)-pleuromutilin (7) (Herzon, 2017). ${ }^{59}$

Herzon's synthesis of (+)-pleuromutilin (7) began with asymmetric copper-catalyzed conjugate addition of dimethylzinc and cyclohexenone (19). Acylation at the $\alpha$-position with Mander's reagent $\mathbf{6 6}^{67}$ and subsequent diastereomeric $\alpha$-methylation ${ }^{68}$ produced 67 in $71 \%$ yield with $>20: 1 \mathrm{dr}$ and $97: 3$ er. Exposure of the freshly prepared $\beta$-ketoester 67 to KHMDS/PhNTf ${ }_{2}$ afforded the corresponding enol triflate (not shown, $88 \%$ yield), which was subjected to palladium-catalyzed carbonylative coupling ${ }^{69}$ (product not shown, 83\% yield). Subsequent $\mathrm{Cu}(\mathrm{OTf})_{2}$-catalyzed Nazarov cyclization $^{70}$ gave cyclopentenone $\mathbf{6 8}$ in $88 \%$ yield. Conjugate addition of
$\mathrm{Et}_{2} \mathrm{AlCN}^{71}$ to cyclopentenone 68 gave adduct 69 with a $3: 1 \mathrm{dr}$; the undesired epimer (not shown) was reduced selectively with DIBAL-H. Immediate treatment of the formed crude product 69 with dilute sodium hydroxide resulted in inversion of the C-4 stereocenter to produce $\mathbf{7 0}$ in $65 \%$ yield. After protection of ketone $\mathbf{7 0}$ as ketal $\mathbf{7 1},{ }^{72}$ addition of excess methyllithium facilitated functionalization of the nitrile moiety in $\mathbf{7 1}$ and subsequent addition of $\mathrm{Boc}_{2} \mathrm{O}$ afforded cyclic enimide $\mathbf{7 4}$ in $80 \%$ yield. The authors proposed that this three-component coupling cascade takes place as follows. Nucleophilic addition of methyllithium to the nitrile group in $\mathbf{7 1}$ produced $\mathbf{7 2}$, which 
underwent lactamization and then deprotonation to give $\mathbf{7 3}$. Boc protection of $\mathbf{7 3}$ produced enimide $\mathbf{7 4 .}$

The organolithium reagent generated in situ from the reaction between iodide $\mathbf{7 5}$ and tert-butyllithium was treated with enimide 74 to provide methyl ketone $\mathbf{7 6}$ in $48 \%$ yield after acid hydrolysis. The authors suggested that electronic activation of the C-14 carbonyl group and minimization of nearby nonbonding interactions by construction of a cyclic enimide functional group were detrimental to the success of this two-fold neopentylic fragment coupling. A three-step transformation from 76 gave aldehyde 77, which was subjected to nickel-catalyzed reductive cyclization, ${ }^{73}$ followed by immediate desilylation to give allylic alcohol 78 in 60\% yield with $>20: 1 \mathrm{dr}$ and $>20: 1$ rr. The authors suggested that the limited number of rotatable bonds along the cyclization precursor 77 reduces the entropic penalty of cyclization and enhances stereo- and regio-control. The $\mathrm{sp}^{2}$-carbons at $\mathrm{C}-10$ and $\mathrm{C}-14$ in the cyclization product 78 can relieve transannular nonbonding interactions in the eightmembered ring, which enables the unprecedented reductive cyclization of a medium-sized ring to be achieved. Oxidation of alcohol 78 and subsequent stereoselective enone reduction with samarium diiodide produced 79 in 98\% yield with $>20: 1$ dr. Single-electron reduction of ketone 79 was accomplished by treatment with sodium in the presence of ethanol. Ketal hydrolysis then afforded $\mathbf{8 0}$ and $\mathbf{8 1}$ in 96\% and $81 \%$ yield, respectively. A two-step transformation from $\mathbf{8 0}$ gave ester $\mathbf{8 2}$, which was subjected to C-12 epimerization via a retro-allylation-allylation process. $^{74}$ Subsequent cleavage of the trityl group afforded (+)-pleuromutilin (7) in 33\% yield and (+)-12epi-pleuromutilin (83) in 56\% yield.

\section{4. (-)-Arcutinine (Qin 2019) $)^{75}$}

Arcutinine (8) is an arcutine-type C20 diterpenoid alkaloid. ${ }^{76}$ It was isolated from Aconitum arcuatam in a 1:2 mixture with arcutine (not shown) by Saidkhodzhaeva and co-workers in $2001 .^{77}$ Shortly after the first enantioselective synthesis of (-)-arcutinine (8), which was reported by Qin and co-workers ${ }^{75}$ in 2019, Li and co-workers reported their asymmetric synthesis of $8 .^{78}$ The enantioselective synthesis of $\mathbf{8}$ reported by Qin is as follows ${ }^{75}$ (Scheme 6). Asymmetric conjugate addition of enone $84^{79}$ with $\mathrm{AlMe}_{3}$ was effected with CuTC/ $(S, S)$-L3 as the catalyst. $^{37}$ The obtained adduct, i.e., aluminum enolate 85 , was trapped by aldehyde 86 to give hydroxy ketone 87 in $45 \%$ yield. Hydrolysis of aluminum enolate 85 gave the corresponding cyclohexanone (not shown) with $92 \%$ ee. A two-step transformation from hydroxy ketone $\mathbf{8 7}$ gave $\mathbf{8 8}$, which was subjected to a sequence of reactions, namely simultaneous ketone and pivalate reduction, Dess-Martin oxidation, selective oxime formation with hydroxylamine, and dehydration with Burgess reagent, to afford nitriles 89 a and 89 b with a $1: 1.5 \mathrm{dr}$ in $43 \%$ yield over four steps. Removal of the MOM group in $89 \mathrm{~b}(\mathrm{R}=$ $\beta-\mathrm{H}$ ) with $\mathrm{TsOH}$ (product not shown, 78\% yield) resulted in selective silyl enol ether formation at C-5 over C-1. The resultant phenol was treated with $\mathrm{TMSCl} / \mathrm{LiI}$ in the presence of HMDS to afford the desired silyl enol ether 90 in $87 \%$ yield with the phenol moiety as the silylated form. Epimerization of 89a to 89b was accomplished by treatment with DBU.

Treatment of 90 with methyllithium and $\mathrm{ClCO}_{2}$ allyl (91) in the presence of HMPA gave a carbonate (not shown, 74\% yield), which was subjected to decarboxylative allylic alkylation/MOM protection of the phenol group (product not shown, 55\% yield). Subsequent palladium-catalyzed olefin isomerization to an internal olefin, ${ }^{80}$ followed by MOM protection of the phenol group, gave cyclohexanone 92 in $71 \%$ yield. A three-step synthesis from 92, which involved simultaneous ketone and nitrile reduction, $N$-tosylation (product not shown, $84 \%$ yield over two steps), and alcohol silylation, gave amine 93 in 91\% yield. The use of the aza-Wacker reaction via Stahl's protocol $^{81}$ converted $\mathbf{9 3}$ to pyrrolidine $\mathbf{9 5}$ as a mixture of diastereomers. The desired isomer, i.e., (-)-95, which was obtained in 54\% yield, was converted to the corresponding ketone 96 in two steps. Cleavage of the MOM group in 96 with TFA, followed by an oxidative dearomatization/intramolecular Diels-Alder reaction cascade of the resulting phenol, produced the cycloaddition adduct 97 in $67 \%$ yield over two steps. Ketylolefin cyclization of freshly prepared 97 , mediated by samarium diiodide, gave the cyclization product 98 in $85 \%$ yield; 98 was subjected to $\alpha$-methylenation (product not shown, 77\% yield) and then reduction to give diol 99 in $77 \%$ yield. Selective installation of an isobutyryl group at the C-15 alcohol of 99 (product not shown, 82\% yield), detosylation effected by Li/naphthalene (product not shown, 93\% yield), and subsequent oxidation of the resultant pyrrolidine with PhIO afforded (-)-arcutinine (8) in $71 \%$ yield along with the undesired isomer (not shown) in 14\% yield.

\subsection{Conidiogenones and conidiogenol (Snyder 2019) ${ }^{82}$}

Conidiogenone B (110), conidiogenone (111), and conidiogenol (9) are tetracyclic diterpenes. They were isolated from fermentation broths and marine-derived endophytic fungi of the Penicillium genus. ${ }^{83,84}$ Biologically, conidiogenone (111) and conidiogenol (9) have potent conidiation-inducing activities, and conidiogenone B (110) shows high antibacterial activity against methicillin-resistant Staphylococcus aureus, Pseudomonas fluorescens, P. aeruginosa, and S. epidermidis. ${ }^{85}$ In 2016, Tu and co-workers disclosed the total syntheses and absolute configurations of 110, 111, and 9. ${ }^{86}$ In 2019, Snyder and co-workers reported the enantioselective syntheses of several conidiogenones $(\mathbf{1 1 0}, \mathbf{1 1 1}, \mathbf{1 1 4}$, and 115) and conidiogenol (9). They described the concept of quaternary-centerguided synthesis of complex polycyclic terpenes ${ }^{82}$ (Scheme 7).

Snyder's syntheses of conidiogenone B (110), conidiogenone (111), and conidiogenol (9) featured asymmetric conjugate addition of enone $\mathbf{1 0 0}$ via Hoveyda's protocol [i.e., with a $\mathrm{Cu}(\mathrm{OTf})_{2} / \mathrm{Ag}-\mathrm{NHC}(\mathrm{Ag}-\mathbf{L 6})$ catalyst $]^{87}$ to give cyclopentane 101 in $79 \%$ yield with $88 \%$ ee $^{82}$ (Scheme 7 ). Conversion of freshly prepared 101 to 102 was accomplished in two steps; 102 was then subjected to Baran's reductive coupling ${ }^{88}$ to give 103 in $80 \%$ yield as a single diastereomer. Corey hydrazine-mediated alkylation $^{89}$ of 103 with iodide 104 afforded an $\alpha$-alkylation product (not shown), which was transformed into the corres- 

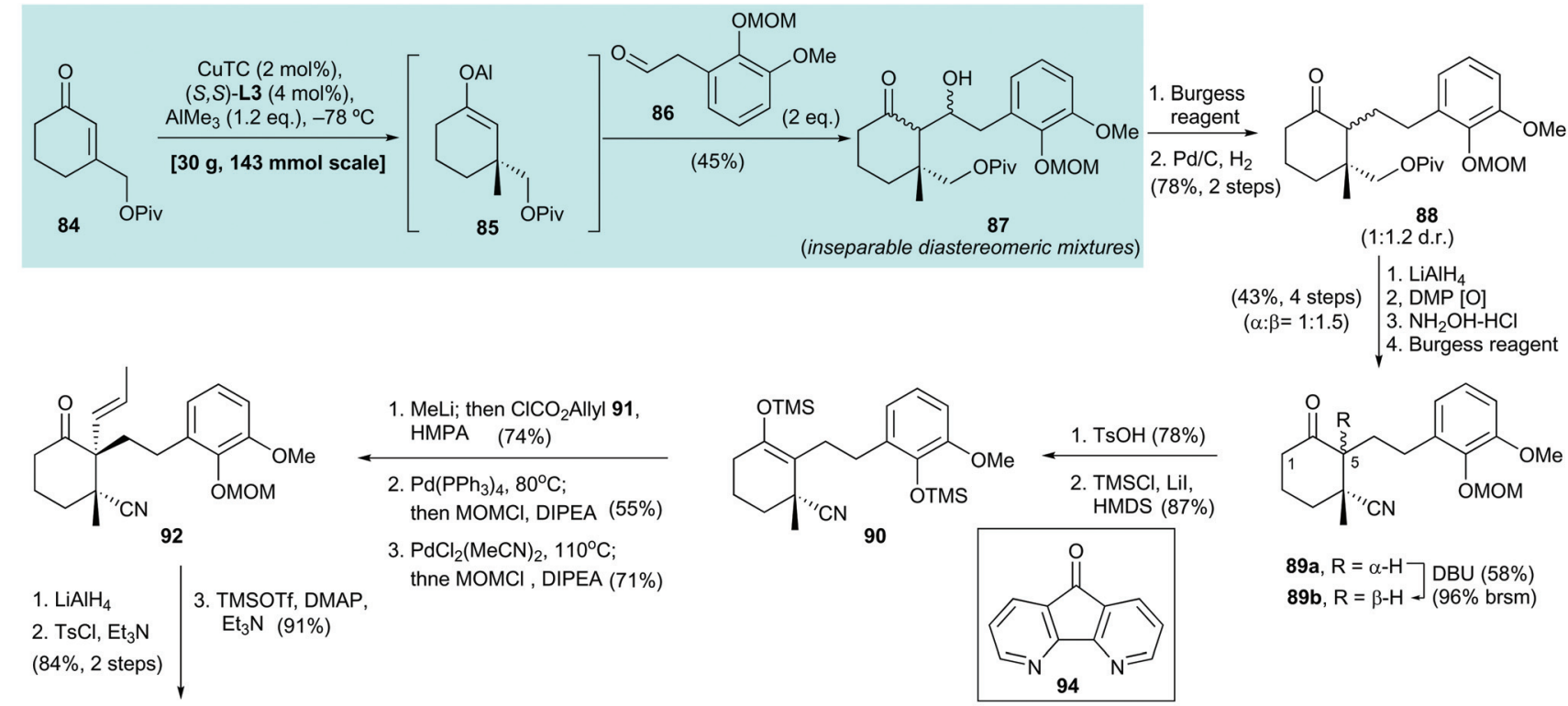

3. $\mathrm{PdCl}_{2}(\mathrm{MeCN})_{2}, 110^{\circ} \mathrm{C}$

thne $\mathrm{MOMCl}$, DIPEA $(71 \%)$

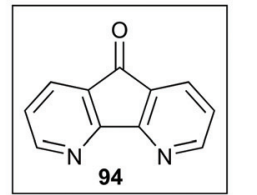

89a, $\mathrm{R}=\alpha-\mathrm{H}]$ DBU (58\%) 89b, $\mathrm{R}=\beta-\mathrm{H}=$ (96\% brsm)

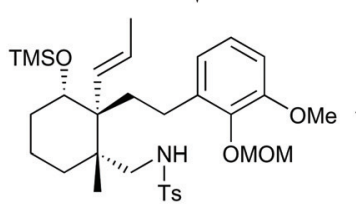

93

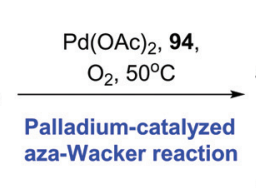

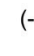

(-) $-95 \mathrm{R}=\alpha-\mathrm{H}(54 \%)$

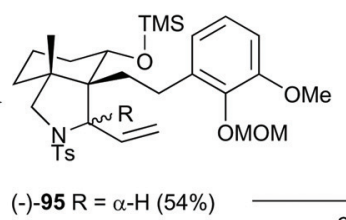

2 steps

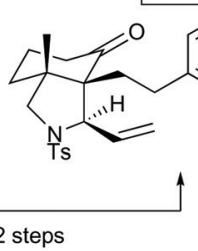

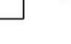

OMOM $\frac{\begin{array}{l}\text { 2. Phl }(\mathrm{OAc})_{2} \\ \text { reflux }\end{array}}{\stackrel{\text { 1. TFA }}{(67 \%, 2 \text { steps })}}$ Oxidative dearomatization/ IMDA cascade

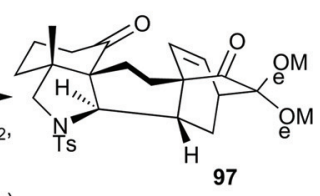

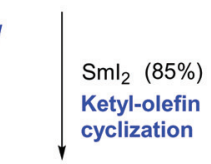

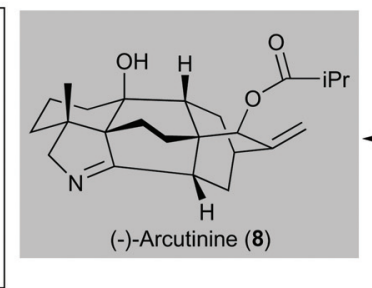

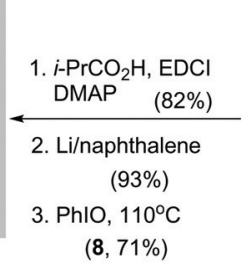

$+14 \%$ undesired isome

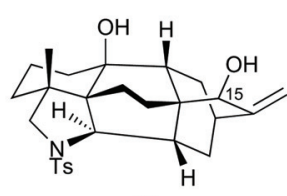

99
1. $\mathrm{CH}_{2}\left(\mathrm{NH}_{2}\right)_{2}$

$\mathrm{Ac}_{2} \mathrm{O}, 100^{\circ} \mathrm{C}$ $(77 \%)$

2. $\mathrm{NaBH}(\mathrm{OMe})_{3}$

$(77 \%)$

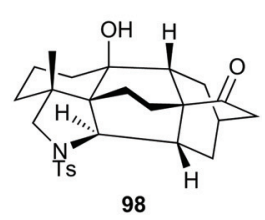

Scheme 6 Enantioselective synthesis of (-)-arcutinine (8) (Qin, 2019). ${ }^{75}$

ponding vinyl triflate 105 by using KHMDS/Comins' reagent. The yield was $61 \%$ over three steps and the rr was >10:1. Cyclization of vinyl triflate $\mathbf{1 0 5}$ was achieved with a palladium catalyst (i.e., $\operatorname{Pd}(\mathrm{OAc})_{2} / t$-BuMePhos) in the presence of $n-\mathrm{Bu}_{4} \mathrm{NOAc}^{90}$ as an oxygen source to give a tricyclic acetate (not shown), which was subjected to saponification to give the corresponding alcohol 106 in 83\% yield as a single diastereomer. Oxidation of alcohol 106 with Dess-Martin periodinane gave an aldehyde (not shown), which was subjected to a radical-based Nozaki-Hiyama-Kishi reaction ${ }^{91,92}$ with diiodide 108. Subsequent silylation gave vinyl iodide 109 in $75 \%$ yield with a $3: 1 \mathrm{dr}$. Reductive Heck coupling ${ }^{93}$ with Herrmann's catalyst ${ }^{94}$ in the presence of $\mathrm{HCO}_{2} \mathrm{Na}$ gave the tetracyclic core (not shown, 70\% yield), which was subjected to catalytic hydrogenation of the olefin. Desaturation with IBX produced conidiogenone B (110) in 71\% yield. Conversion of 110 to conidiogenone (111) and conidiogenol (9) was achieved by using Tu's reported conditions. ${ }^{86}$
In the same work, Snyder and co-workers synthesized conidiogenones C (114) and D (115) ${ }^{82}$ (Scheme 7B). Treatment of 102 with $m$-CPBA and cyclization with $\mathrm{Cp}_{2} \mathrm{TiCl}_{2}{ }^{95}$ produced 112 and 113, which were used in an eight-step synthesis to give conidiogenone $\mathrm{C}$ (114) and conidiogenone D (115), respectively.

\section{6. (+)-Waihoensene (Yang $2020 ;{ }^{96}$ Snyder $2020{ }^{97}$ )}

Waihoensene (10) is a tetracyclic diterpene; it was isolated from the New Zealand podocarp Podocarpus totara var waihoensis by Weavers and co-workers in $1997 .{ }^{98}$ After the first racemic synthesis of 10 by Lee and co-workers in $2017,{ }^{99}$ the asymmetric synthesis of $(+)$-waihoensene (10) was reported by Yang's group $^{96}$ and Snyder's group ${ }^{97}$ independently in 2020. A copper-catalyzed asymmetric conjugate reaction was the key synthetic step in each synthesis (Scheme 8).

In Yang's synthesis, ${ }^{96}$ asymmetric conjugate addition of trimethylaluminum and $\mathbf{1 1 6}$ with $\mathrm{CuTC} /(R, R)-\mathbf{L} 3$ as the cata- 
A.
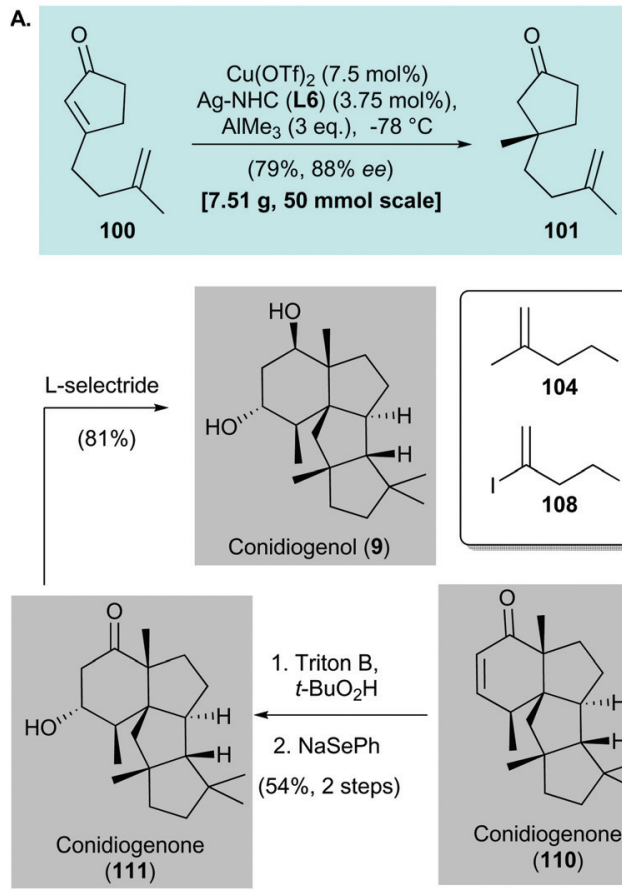
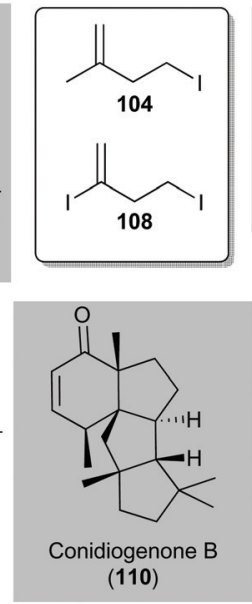

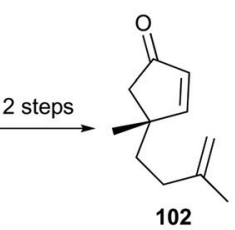

102

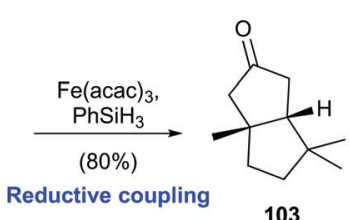

103

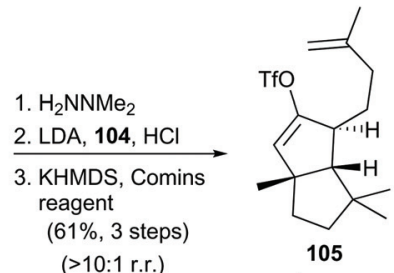

$(>10: 1$ r.r.)
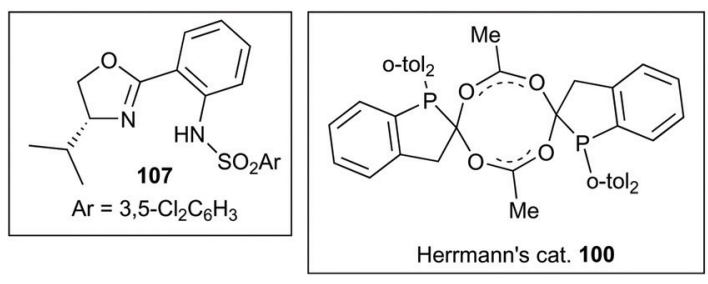

1,3-trans

Heck/

$n-\mathrm{Bu}_{4} \mathrm{NOAc}$;

$n-\mathrm{Bu}_{4} \mathrm{NOAc}$;
then $\mathrm{K}_{2} \mathrm{CO}_{3}$,

(83\%)

\section{Herrmann's cat. $\mathbf{1 0 0}$} $\mathrm{HCO}_{2} \mathrm{Na}$, then TBAF $(70 \%)$

Reductive Heck coupling

2. $\mathrm{Pd} / \mathrm{C}, \mathrm{H}_{2}$; then IBX

(71\%)
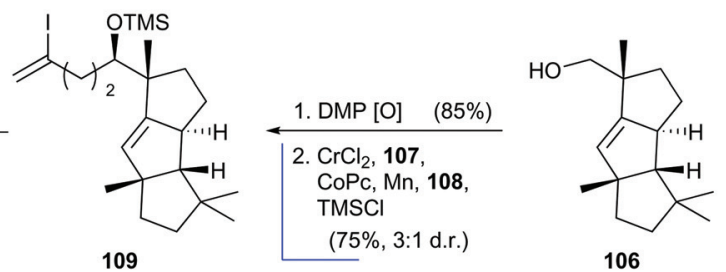

Hindered NHK reaction

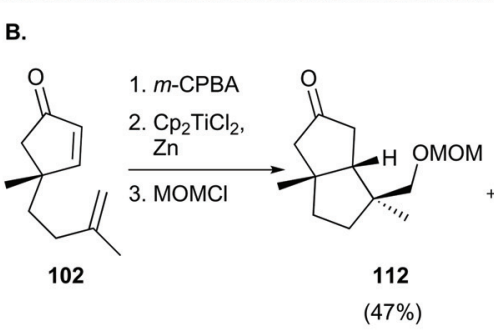

(47\%)

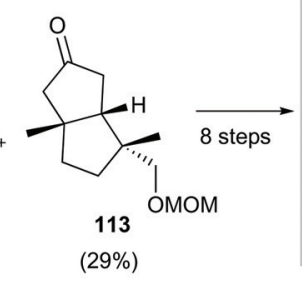

$(29 \%)$

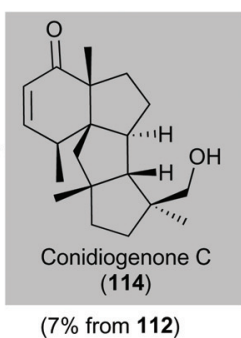

(7\% from 112)
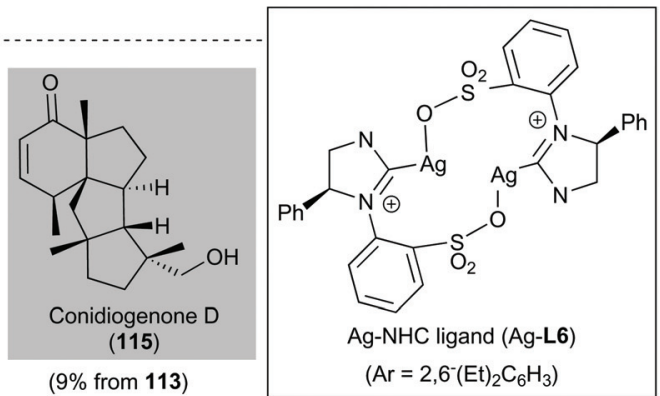

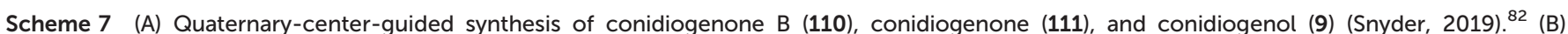
Synthesis of conidiogenones C (114) and D (115) achieved in the same work via a similar synthetic approach. ${ }^{82}$

lyst, ${ }^{100}$ followed by $\alpha$-methylenation, afforded enone 118 in $61 \%$ yield with $99 \%$ ee (Scheme $8 \mathrm{~A}$ ). Conversion of enone 118 to diyne 119 was accomplished in three steps. Diyne 119 was subjected to a $t$-BuOK-catalyzed Conia-ene type cyclization ${ }^{101}$ to give enyne 120 in $83 \%$ yield. A Pauson-Khand reaction ${ }^{102}$ of enyne 120 mediated by $\mathrm{Co}_{2}(\mathrm{CO})_{8}$ in the presence of $\mathrm{N}_{2} \mathrm{O}^{103}$ gave cyclized enone 121 in 59\% yield with 93\% ee. This was subjected to nickel-catalyzed methylation ${ }^{104}$ to produce diketone 122 in $81 \%$ yield as a single diastereomer. A three-step synthesis from diketone 122 gave ketone 123, which was subjected to diastereoselective hydrogenation through radicalmediated hydrogen-atom transfer ${ }^{88}$ to give $\mathbf{1 2 4}$ in $75 \%$ yield. $\alpha$-Methylation of $\mathbf{1 2 4}$ (product not shown, 90\% yield) and then a Wittig reaction gave (+)-waihoensene (10) in $90 \%$ yield.

In Snyder's synthesis of (+)-waihoensene (10), ${ }^{97}$ asymmetric conjugate addition of trimethylaluminum and cyclopentenone 125 via Hoveyda's protocol, with $\mathrm{Cu}(\mathrm{OTf})_{2} / \mathrm{Ag}-\mathrm{NHC}$ ligand (AgL6) as the catalyst, ${ }^{47,105}$ afforded cyclopentanone 126 in $81 \%$ yield with $92 \%$ ee (Scheme $8 \mathrm{~B}$ ). Treatment of cyclopentanone
126 with NaHMDS and subsequent trapping of the enolate intermediate with Mander's reagent 66 afforded 127b and 127a in a 1.8:1 ratio and $88 \%$ combined yield. The authors suggested that the potential regioselectivity observed on treatment with a base (i.e., NaHMDS) can be ascribed to a weak directing effect by the alkyne. ${ }^{82,106}$ The undesired isomer $127 \mathbf{a}$ was recycled to $\mathbf{1 2 6}$ by Krapcho decarboxylation. ${ }^{107}$ Desilylation of $\mathbf{1 2 7} \mathbf{b}$, followed by a Conia-ene reaction in the presence of catalytic amounts of $\mathrm{Ph}_{3} \mathrm{PAuCl} / \mathrm{AgOTf},{ }^{108}$ afforded alkene 128 in 94\% yield over two steps. Catalytic hydrogenation of 128 with $\mathrm{PtO}_{2}$ as the catalyst provided 129 in 76\% yield with a $3.2: 1 \mathrm{dr}$. Enyne 130 was obtained in six steps from 129. A Pauson-Khand reaction of 130 at $160{ }^{\circ} \mathrm{C}$ afforded the tetracyclic enone $131^{99}$ in $50 \%$ yield. The authors mentioned that a high reaction temperature is critical for a successful reaction because it is likely to increase the reaction rate and overcome the energy barrier required for quaternary center formation. ${ }^{96}$ A three-step synthesis from 131 afforded (+)-waihoensene (10). 
A.
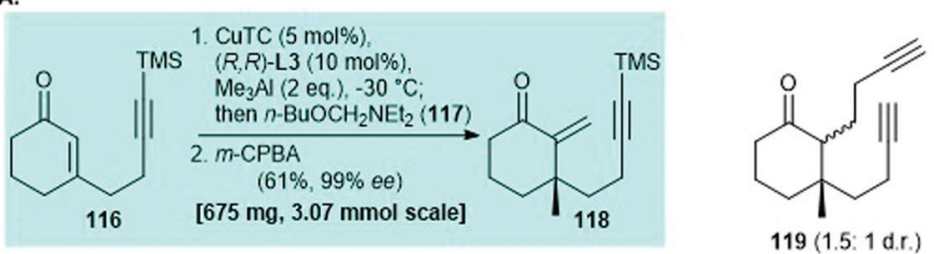

\begin{tabular}{|l|} 
1. AllyITMS, $\mathrm{BF}_{3} \mathrm{OEt}_{2}(89 \%)$ \\
2. $\mathrm{O}_{3}$; then $\mathrm{PPh}_{3} \quad(65 \%)$
\end{tabular}

3. Bestmann reag., $\mathrm{K}_{2} \mathrm{CO}_{3}(72 \%)$

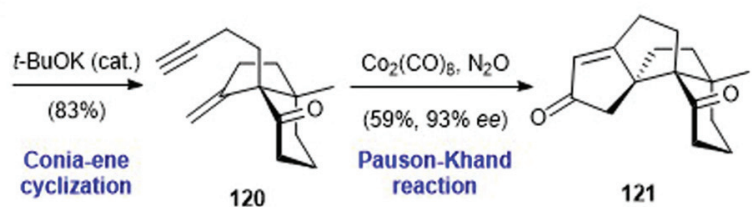

120

reaction

121
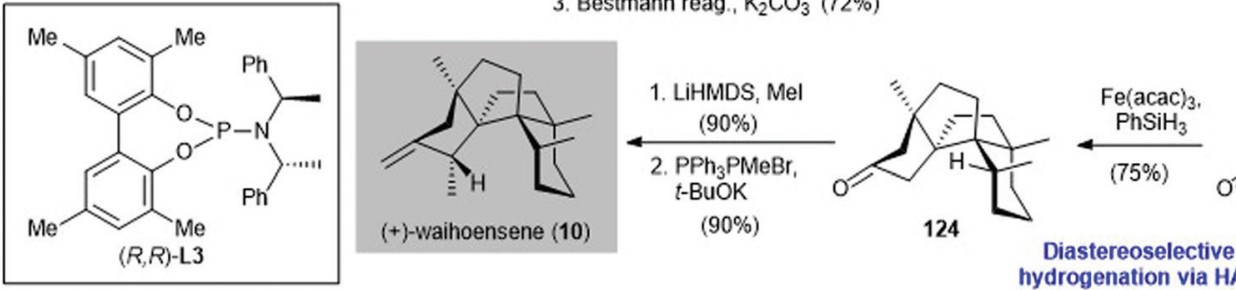

hydrogenation via HAT

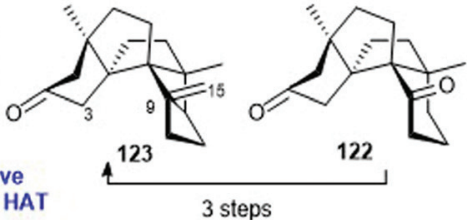

B.
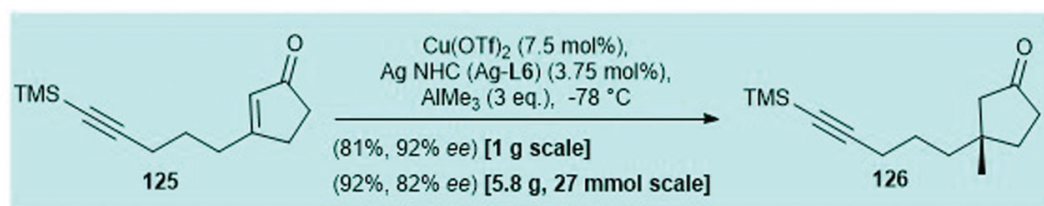

NaHMDS, 66

$(88 \%)$

$(127 \mathrm{~b}: 127 \mathrm{a}=1.8: 1)$

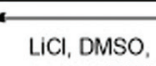

$150^{\circ} \mathrm{C}(76 \%)$
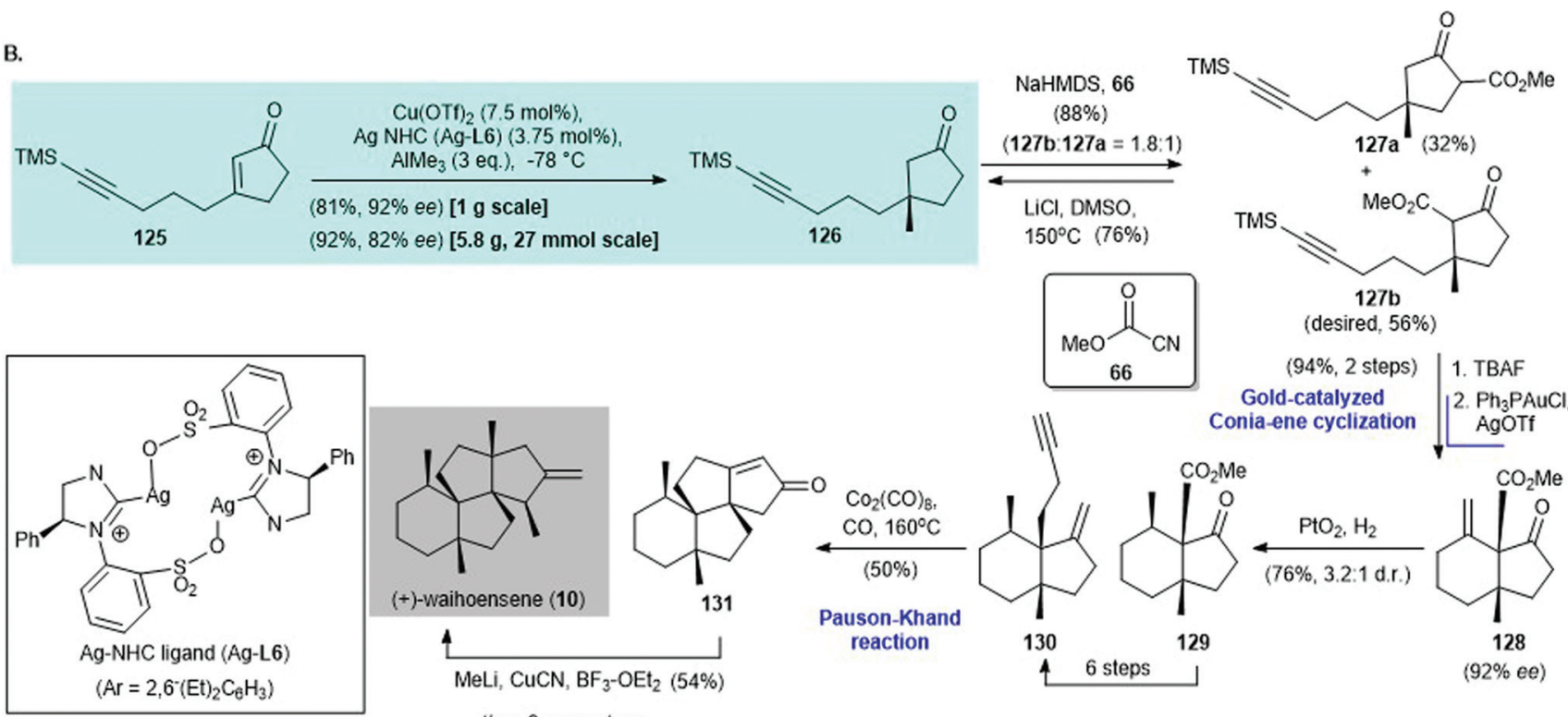

MeLi, CuCN, $\mathrm{BF}_{3}-\mathrm{OEt}_{2}$ (54\%)

Pauson-Khand reaction

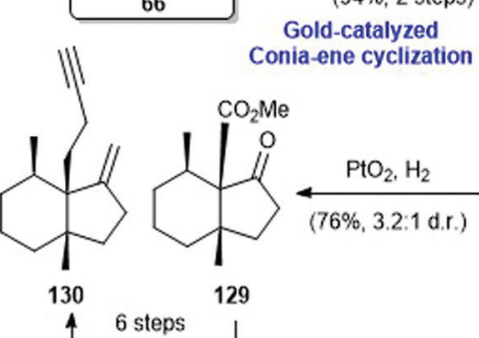

1. TBAF $\sqrt{\begin{array}{l}\text { 2. } \mathrm{Ph}_{3} \mathrm{PAuCl} \\ \mathrm{AgOTf}\end{array}}$

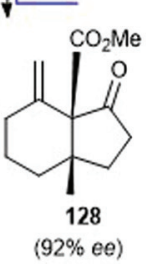

then 2 more steps

Scheme 8 Enantioselective synthesis of (+)-waihoensene (10) reported independently by (A) Yang (2020) ${ }^{96}$ and (B) Snyder (2020). ${ }^{97}$

\subsection{Taxol® $\left(\right.$ Baran 2020) ${ }^{109}$}

The prominent biological profile of Taxol® (11) has made it an important synthetic target since $1994 .{ }^{110-120}$ The concept of two-phase terpene synthesis was introduced by Baran and coworkers in $2009^{121,122}$ Since then, the same laboratory has focused on the total synthesis of $\mathbf{1 1}$ via the two-phase approach. ${ }^{30,123,124}$ In 2020, Baran and co-workers achieved the total synthesis of $\mathbf{1 1}$ by using a two-phase approach ${ }^{109}$ (Scheme 9). The previously achieved enantioselective synthesis of (+)-taxadienone (1) featured a copper-catalyzed asymmetric conjugate addition to build the 6-8-6 tricyclic core 24 with $>99: 1 \mathrm{dr}$ (see Scheme 2A); 24 is also an intermediate in Baran's Taxol® synthesis. In a new version of the method for preparing tricycle $\mathbf{2 4}$, a copper-catalyzed asymmetric conjugate addition of $\mathrm{MeMgBr}$ as the alkylating agent to enone 21 was effected by the chiral NHC ligand $\mathbf{L 7} .^{125}$ Enolate trapping with acrolein 23 gave triene 132 after oxidation with Dess-Martin periodinane. An intramolecular Diels-Alder reaction of triene 132, mediated by $\mathrm{BF}_{3}-\mathrm{OEt}_{2}$, gave the desired tricycle 24 in $10.6 \%$ yield from enone 21 . The complex framework 24 is regarded as the end-product of the cyclase phase.

With the cyclase phase end-product $\mathbf{2 4}$ in hand, strategic oxidation of the cyclic framework completed the terpene (in this case Taxol®) synthesis. This is called the oxidase phase. Selective allylic oxidation at the C-13 position was achieved by using the $\mathrm{Cr}(\mathrm{v})$-based oxidant $133^{124,126}$ and HFIP: TMSOH $(2: 1)$ as the solvent. Selective bromination with $\mathrm{CuBr}_{2}$ at the C-5 position gave triketone 134 in $55 \%$ yield over two steps. Allylic bromination of $\mathbf{1 3 4}$ with NBS/BPO at the C-10 position gave a C-5/C-10 dibromide (not shown). Subsequent radicalbased oxidation ${ }^{124}$ mediated by $\mathrm{Ag}(\mathrm{I})$ selectively replaced the 


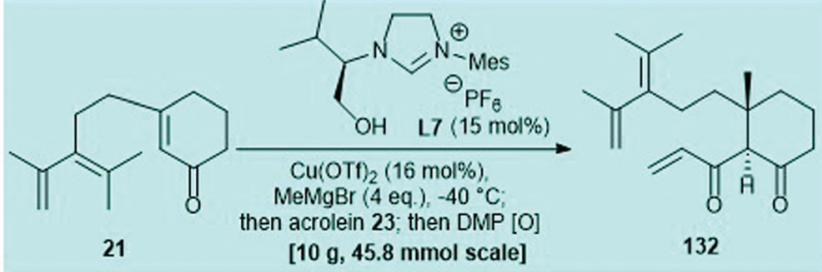

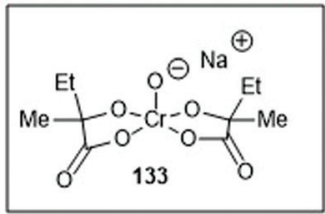

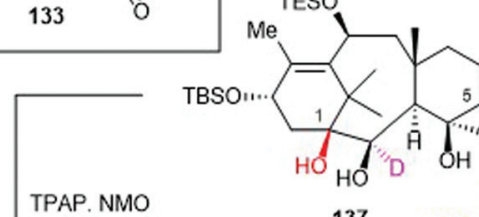

$(81 \%)$

137

Selective epoxidation/

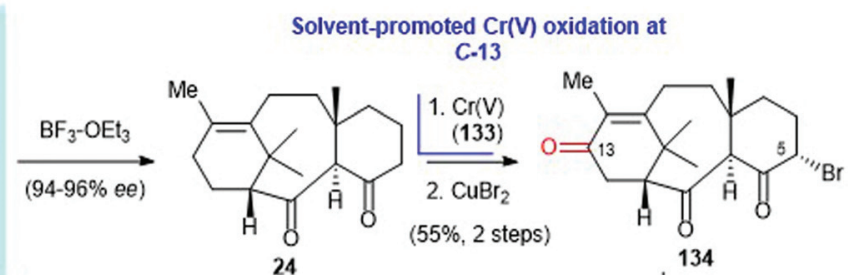

(10.6\% yield from 21)

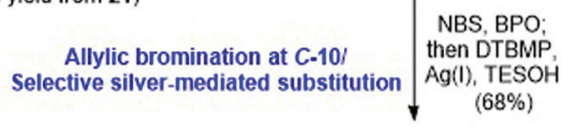
$(68 \%)$

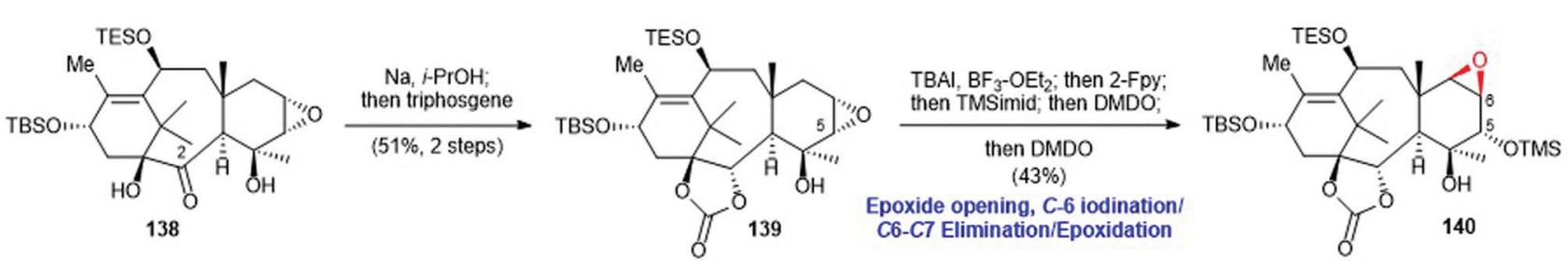

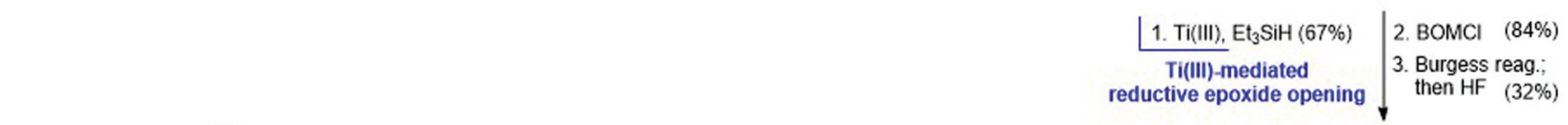

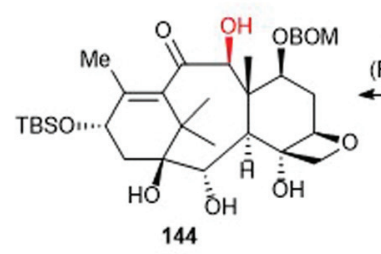

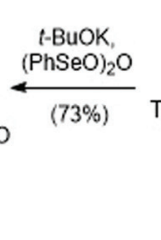

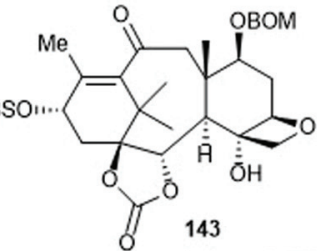

143 DIPEA; then IBX

(62\%)

$(60 \%) \quad \begin{gathered}t-\text { BuOK; then } \text { triphosgene; } \\ \text { then } \mathrm{Ac}_{2} \mathrm{O} \\ \text { Redox isomerization }\end{gathered}$

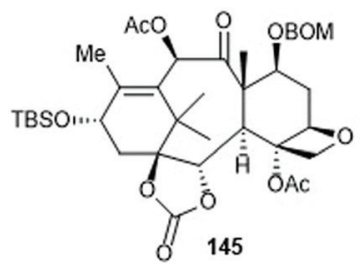
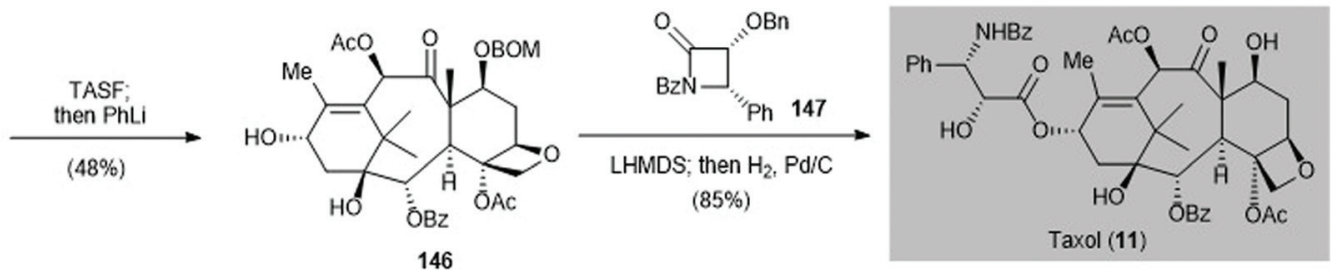

Scheme 9 Synthesis of Taxol® (11) via a two-phase synthetic approach (Baran, 2020). ${ }^{109}$ (Newly introduced oxygen atom(s) in the synthetic step is/ are highlighted in red).

C-10 bromide by TESOH to give 135 in $68 \%$ yield. Deuterated 136 was obtained in three steps from triketone 135; these steps were elimination of the $\mathrm{C}-5$ bromide using $\mathrm{LiBr}, 1,2$-addition to the $\mathrm{C}-4$ ketone using $\mathrm{MeMgBr}$, selective reduction of the C-13 ketone with DIBAL-H, reduction of the C-2 ketone with LiAlD $_{4}$, and selective silylation of the C-13 hydroxy group with
TBSCl to give 136. Chemo- and stereo-selective oxidation of the deuterated alcohol 136 with DMDO afforded the epoxy-triol 137 in $49 \%$ yield. $^{127}$ It was subjected to TPAP-mediated ${ }^{128}$ oxi- $^{-}$ dation to give ketone 138 in $81 \%$ yield. The C-11-C-12 olefin in 136 is shielded by the $\mathrm{C}-10$ and $\mathrm{C}-13$ substituents, and is therefore protected from DMDO oxidation. ${ }^{129,130}$ The deuterium 
atom at the C-2 position of $\mathbf{1 3 6}$ promotes chemoselective oxidation at C-1 via the kinetic isotopic effect ${ }^{131}$ and simultaneously acts as a blocking group ${ }^{132}$ to prevent oxidation of the C-2 hydroxy group to the corresponding ketone.

Thermodynamic reduction of the C-2 carbonyl group of $\mathbf{1 3 8}$ by $\mathrm{Na} / \mathrm{i}-\mathrm{PrOH}$ and then exposure to triphosgene produced carbonate 139 in $51 \%$ yield. This was subjected to a sequence of reactions: (i) selective epoxide opening of 139 with $\mathrm{BF}_{3}-\mathrm{OEt}_{2}$ and iodination at the C- 6 position, followed by addition of 2-fluoropyridine (2-Fpy) to sequester the boron salt, gave an iodide (not shown), (ii) immediate protection of the C-5 hydroxy group from epoxide opening with TMS-imidazole and oxidation of the C- 6 iodide by DMDO, which led to elimination and generated a silylated alcohol with a $\mathrm{C}=\mathrm{C}$ double bond at the C-6 and C-7 positions (not shown) and (iii) further oxidation by DMDO to generate epoxytaxane 140 in $43 \%$ overall yield. The regioselective reductive opening of $\mathbf{1 4 0}$ was sterically guided by $\mathrm{Ti}(\mathrm{III})^{133}$ in the presence of $\mathrm{Et}_{3} \mathrm{SiH}$ to give an alcohol (not shown, 67\% yield), which was protected by BOMCl (not shown, 84\% yield); subsequent Burgess dehydration produced 141 in 32\% yield. Mesylation of 141 and then dihydroxylation with $\mathrm{OsO}_{4}$ gave 142 in 68\% yield. This was treated with a hindered amine base (i.e., diisopropylethylamine) to give an oxetane and subsequent exposure to IBX produced enone 143 in $62 \%$ yield. $\alpha$-Hydroxylation of enone 143 with $t$-BuOK/(PhSeO $)_{2} \mathrm{O}$ gave 144 in $73 \%$ yield, with unintended cleavage of the cyclic carbonate. Redox isomerization of 144 by treatment with $t$-BuOK and acylation with $\mathrm{Ac}_{2} \mathrm{O}$ regenerated the cyclic carbonate and acetylated C-4/C-10 simultaneously to produce taxane 145 in $60 \%$ yield. Desilylation of 145, followed by treatment with $\mathrm{PhLi}$, gave BOM-group-bearing baccatin III 146 in $48 \%$ yield. ${ }^{115}$ Subsequent Ojima acylation ${ }^{134}$ with $\beta$-lactam 147 in the presence of the lithium alkoxide of 146, followed by catalytic hydrogenation, produced Taxol ${ }^{\circledR}(\mathbf{1 1})$ in $85 \%$ yield.

\section{Construction of all-carbon quaternary stereocenters by palladium-catalyzed asymmetric conjugate addition}

Palladium-catalyzed asymmetric conjugate addition of arylboronic acids to $\beta$-substituted cyclic enones was reported by Stoltz's group $^{135}$ and Minnaard's group ${ }^{136}$ in 2011 and 2012, respectively, shortly after Lu's report of a racemic version in $2010^{137}$ (Scheme 10). In Stoltz's work, asymmetric conjugate addition of phenylboronic acid (148) to 3-methylcyclohex-2enone (25), with catalytic amounts of $\mathrm{Pd}\left(\mathrm{OCOCF}_{3}\right)_{2}$ and $(S)-t$ BuPyOx (L8) as the chiral ligand, afforded cyclohexanone 149 in $99 \%$ yield with $93 \%$ ee $^{135}$ (Scheme 10A). Later in 2013, the same group suggested a possible mechanism for this elegant method $^{138}$ (Scheme 10B). The catalytic cycle begins with transmetalation of $\mathbf{1 4 8}$ with the cationic palladium catalyst $\mathbf{A}$ to give the cationic arylpalladium intermediate $\mathbf{B}$. Coordination of
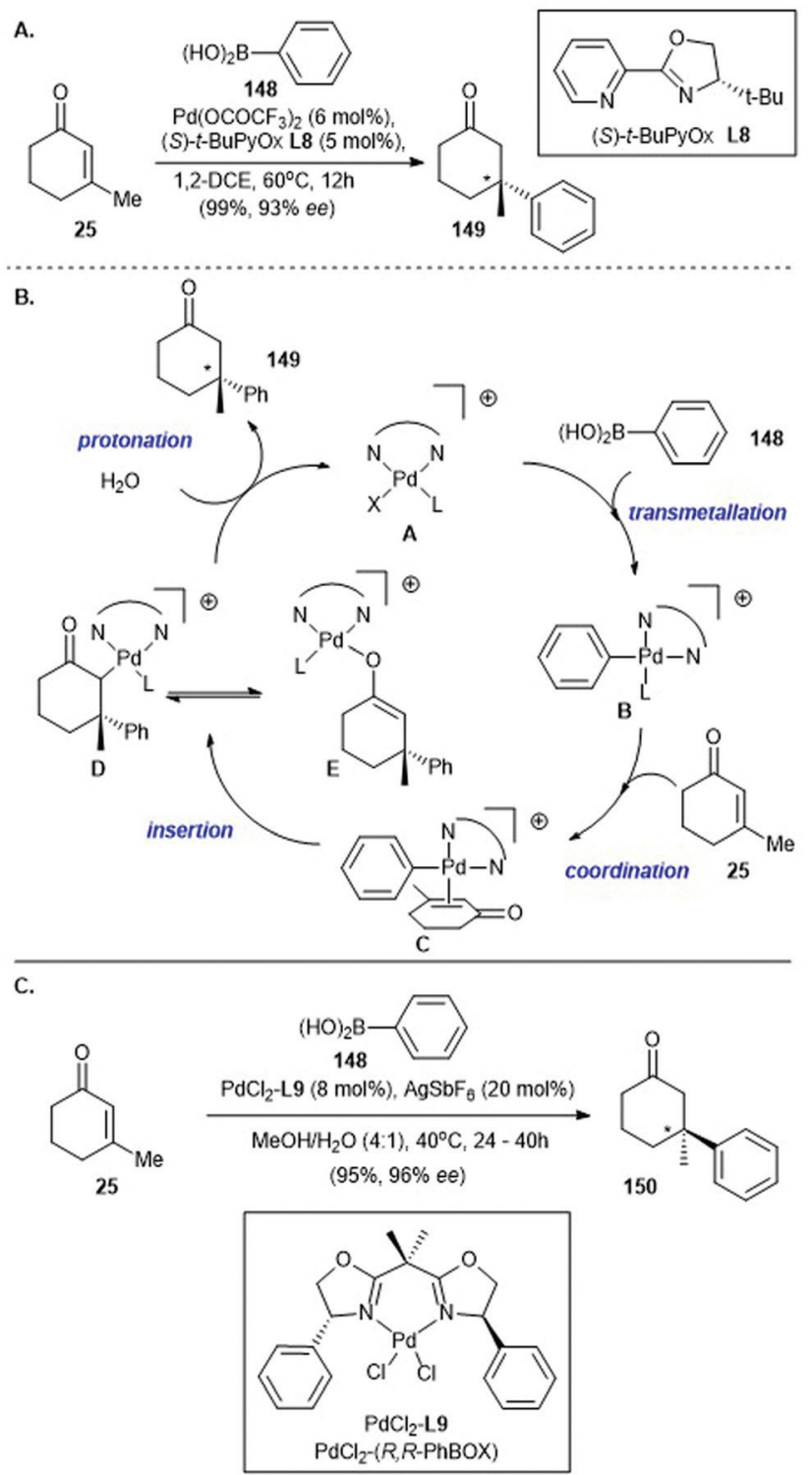

Scheme 10 (A) Palladium-catalyzed asymmetric conjugate addition of arylboronic acid 148 to $\beta$-substituted cyclic enone 25 (Stoltz, 2011). ${ }^{135}$ (B) Proposed catalytic cycle reported by the same group in $2013 .{ }^{138}$ (C) Palladium catalyzed asymmetric conjugate addition of arylboronic acid 148 to $\beta$-substituted cyclic enone 25 , effected by the $\mathrm{PdCl}_{2}(R, R-\mathrm{PhBox})$ ( $\mathrm{PdCl}_{2}$ L9) catalyst (Minnaard, 2012). ${ }^{136}$

enone 25 to arylpalladium intermediate $\mathbf{B}$ produces a cationic $\pi$-complex $\mathbf{C}$, which undergoes rate- and enantioselectivitydetermining insertion of the aryl moiety into the enone $\pi$-system to give carbon-bound palladium enolate $\mathbf{D}$, which either tautomerizes to the corresponding oxygen-bound palladium enolate $\mathbf{E}$ or undergoes protonation to give the enantioenriched conjugate addition product 149. Regeneration of the cationic palladium catalyst A completes the catalytic cycle.

In 2012, Minnaard and co-workers disclosed the palladiumcatalyzed asymmetric conjugate addition of an arylboronic acid to a $\beta$-substituted cyclic enone by using a $\operatorname{PdCl}_{2}(R, R$ - 
A.
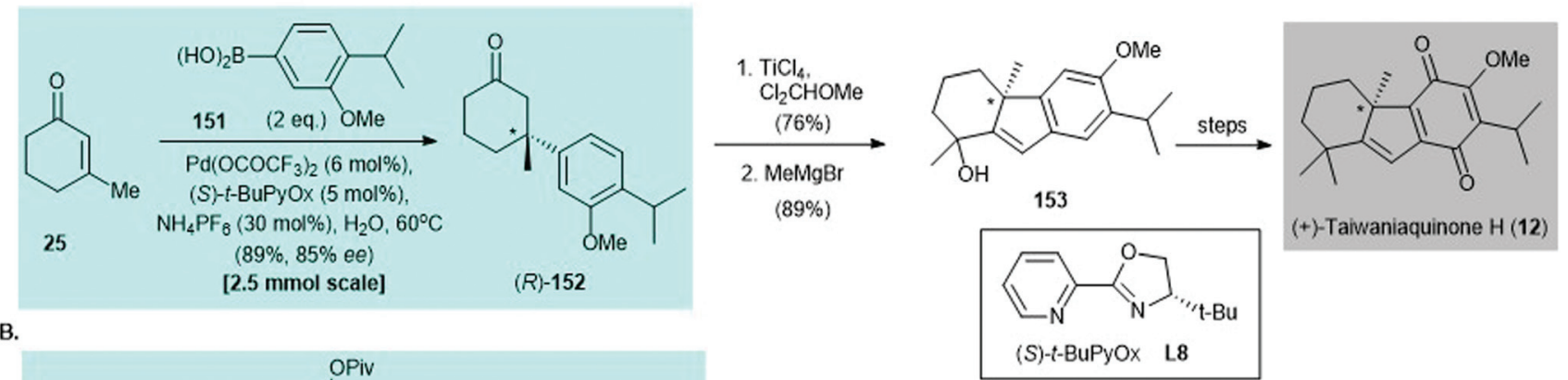

B.
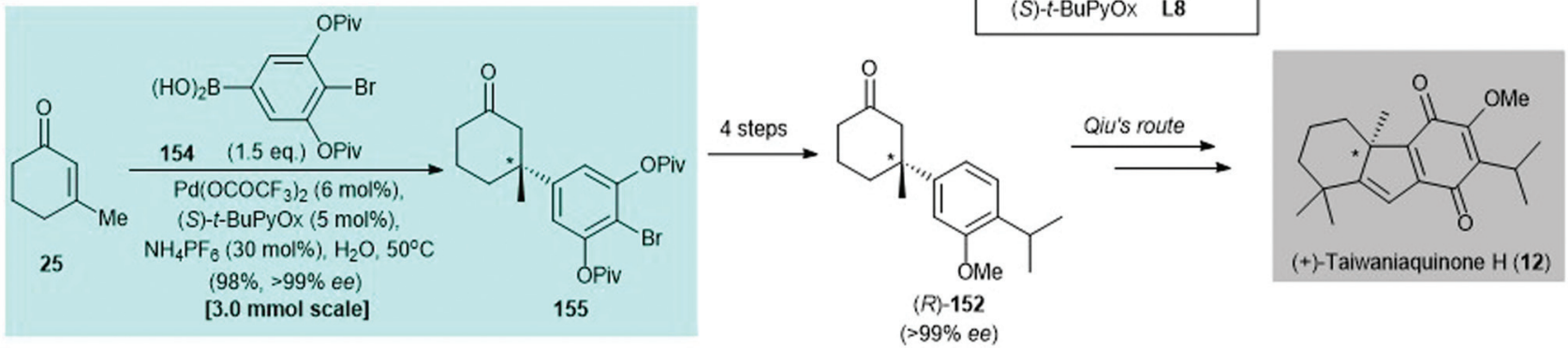

C.
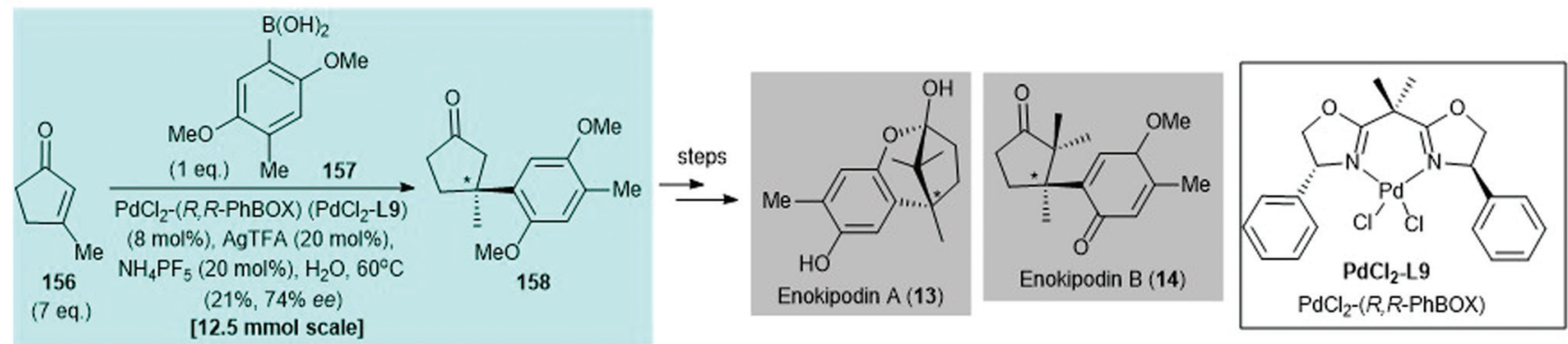

Scheme 11 Selected examples of construction of all-carbon quaternary stereocenters by palladium-catalyzed asymmetric conjugate addition of arylboronic acid to enone in natural product syntheses reported before 2016. (A) Synthesis of (+)-taiwaniaquinone H (12) (Qin, 2014). ${ }^{139}$ (B) Another synthesis of (+)-taiwaniaquinone H (12) (Stoltz, 2014) ${ }^{140}$ (C) Formal syntheses of enokipodin A (13) and enokipodin B (14) (Minnaard, 2014). ${ }^{143}$

PhBox) ( $\left.\mathrm{PdCl}_{2} \mathbf{L} 9\right)$ catalyst and $20 \mathrm{~mol}^{\circ} \mathrm{AgSbF}_{6}$ as an additive $^{136}$ (Scheme 10C). Under the optimized conditions, asymmetric conjugate addition of phenylboronic acid (148) to 3-methylcyclohex-2-enone (25) gave the conjugate addition adduct 150 in $95 \%$ yield with $96 \%$ ee. These elegant methods reported by Stoltz ${ }^{135}$ and Minnaard, ${ }^{136}$ which enable the efficient construction of $\beta$-aryl-substituted all-carbon quaternary stereocenters, have been used in natural product synthesis.

Synthesis of (+)-taiwaniaquinone $\mathrm{H}$ (12) was accomplished by Qin $^{139}$ and Stoltz ${ }^{140}$ independently in 2014 (Scheme 11A and B). The palladium-catalyzed asymmetric conjugate addition of arylboronic acids to enones, which was developed by Stoltz and co-workers in $2011,{ }^{135}$ features as the key reaction in both syntheses. In Qin's work, ${ }^{139}$ catalytic asymmetric conjugate addition of arylboronic acid $\mathbf{1 5 1}$ to enone 25 afforded the enantioenriched conjugate adduct $(R)-\mathbf{1 5 2}$ in $89 \%$ yield with $85 \%$ ee. The synthesis of (+)-taiwaniaquinone $\mathrm{H}(\mathbf{1 2})$ was completed by using a reported procedure. ${ }^{141,142}$ In Stoltz's synthesis, ${ }^{140}$ palladium-catalyzed asymmetric conjugate addition of arylboronic acid 154 to enone 25 gave aryl bromide 155 in 98\% yield with $>99 \%$ ee; 155 was converted to Qin's intermediate $(R)-\mathbf{1 5 2}$ in four steps. ${ }^{139}$ The synthesis of (+)-taiwaniaqui- none $\mathrm{H}$ (12) was achieved by using a reported protocol. ${ }^{139}$ The preparation of (+)-dichroanone (not shown) was also accomplished in studies by Qin $^{139}$ and Stoltz ${ }^{140}$ but is not described here.

In 2014, Minnaard and co-workers reported the enantioselective synthesis of enokipodin A (13) and enokipodin B (14) via asymmetric conjugate addition of arylboronic acid 148 to 3-methyl-2-pentenone (156) with $\mathrm{PdCl}_{2}\left(R, R\right.$-PhBOX) $\left(\mathrm{PdCl}_{2} \mathbf{L 9}\right)$ as the catalyst. ${ }^{143}$ The enantioenriched conjugate 158 was obtained in $21 \%$ yield with $74 \%$ ee $^{143}$ (Scheme 11C). The synthesis of herbertenediol (not shown) was also disclosed in the same work but is not discussed here.

\section{1. (+)-Triptolide and (-)-triptophenolide (Qin 2016) ${ }^{144}$}

Triptolide (15) is a potent antitumor and immunosuppressive agent. It was isolated by Kupchan and co-workers from the Chinese medicinal plant Tripterygium wilfordii Hook $\mathrm{F}$ in 1972. ${ }^{145}$ In 2016, Qin and co-workers disclosed the formal syntheses of (+)-triptolide (15) and (-)-triptophenolide (16) ${ }^{144}$ (Scheme 12). The enantioenriched conjugate adduct 160 was prepared by asymmetric conjugate addition of arylboronic acid 159 to enone 25 by using Stoltz's protocol; ${ }^{135} 160$ was isolated 


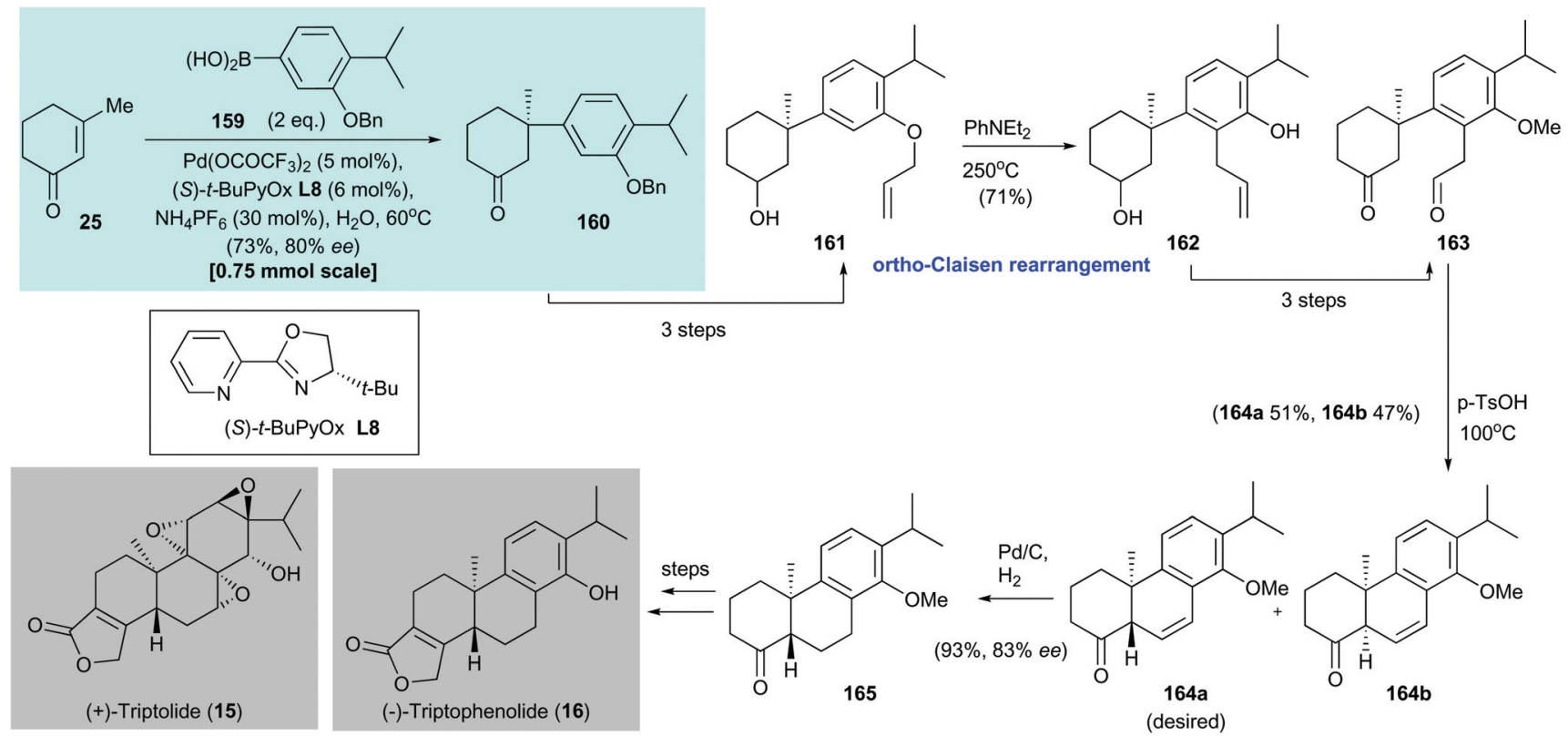

Scheme 12 Formal syntheses of (+)-triptolide (15) and (-)-triptophenolide (16) (Qin, 2016). ${ }^{144}$

in $73 \%$ yield with $80 \%$ ee. Allyl ether 161 , which was synthesized in three steps from conjugate addition adduct 160, underwent an ortho-Claisen rearrangement on heating to afford phenol 162 in 71\% yield. Conversion of freshly prepared phenol 162 to aldehyde 163 was accomplished in three steps. An acid-catalyzed intramolecular aldol condensation of 163 produced a mixture of $164 \mathrm{a}$ ( $51 \%$ yield) and $164 \mathrm{~b}$ ( $47 \%$ yield). Catalytic hydrogenation of the major isomer 164a gave 165 in $93 \%$ yield with $83 \%$ ee. This is the precursor for synthesizing (+)-triptolide (15) and (-)-triptophenolide (16). ${ }^{146}$

\subsection{Mastigophorene A (Minnaard and Feringa $2016^{147}$ )}

Mastigophorene A (17) is a dimeric sesquiterpene. It was isolated from the liverwort Mastigophora diclados by Asakawa and co-workers in $1988 .{ }^{148}$ It shows neurotrophic activity at concentrations as low as $0.1-1 \mu \mathrm{M} .^{149,150}$

In 2016, Minnaard and Feringa reported the atroposelective total synthesis of mastigophorene A (17) via palladium-catalyzed, tert-butyllithium-mediated dimerization of an aryl halide (Scheme 13). ${ }^{147}$ The preparation of mastigophorene A

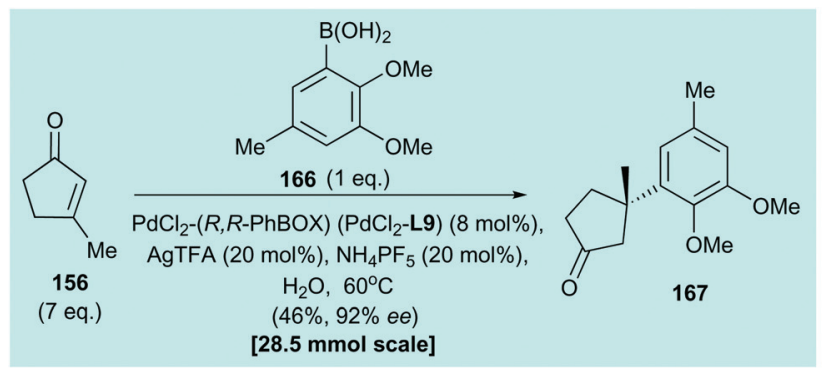

[28.5 mmol scale]
$\mathrm{Pd}\left(\mathrm{CF}_{3} \mathrm{CO}_{2}\right)_{2}(40 \mathrm{~mol} \%)$ $168(40 \mathrm{~mol} \%), \mathrm{O}_{2}(1 \mathrm{~atm})$ $(72-79 \%)$

Palladium-catalyzed aerobic $\alpha, \beta$-dehydrogenation

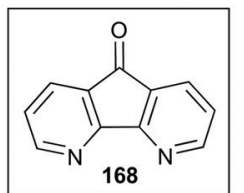<smiles>COc1cc(C)cc([C@]2(C)C=CC(=O)C2)c1OC</smiles>
169<smiles>COc1cc(C)cc([C@]2(C)CCC[C@@]2(C)O[Na])c1OC</smiles><smiles></smiles>
pyridinium tribromide $(96 \%)$ or $\mathrm{Br}_{2}(98 \%)$
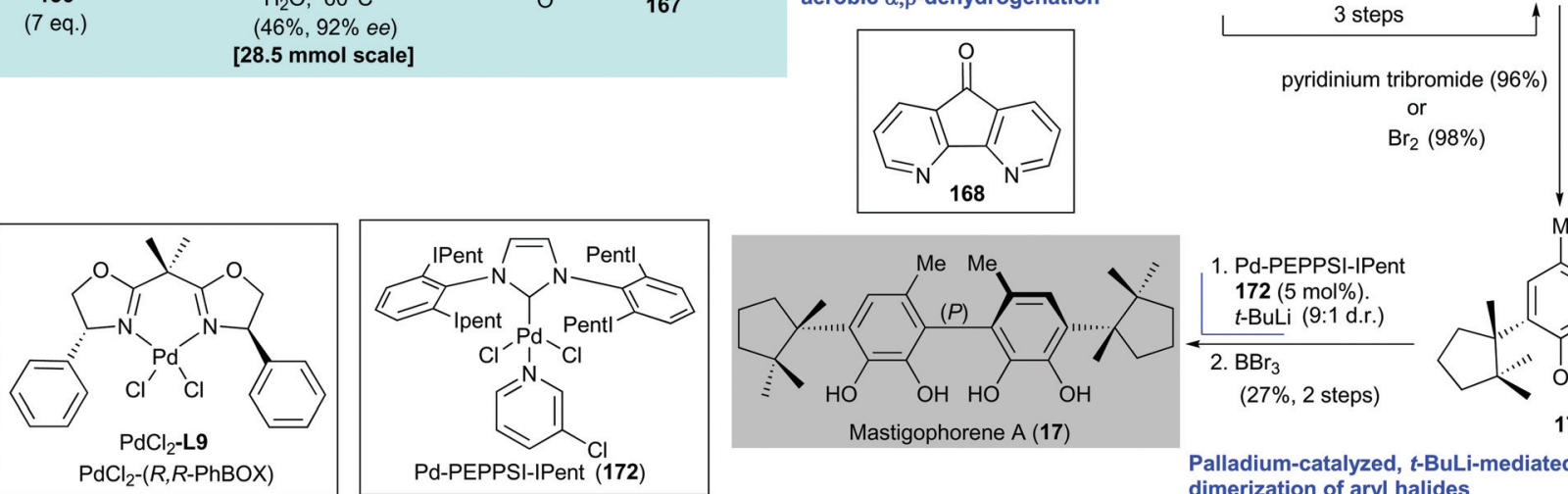
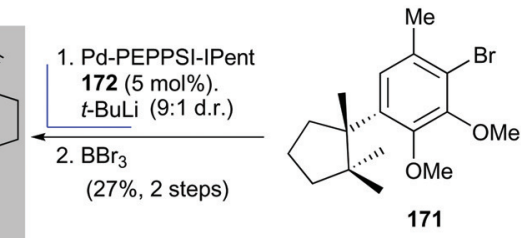

Palladium-catalyzed, $t$-BuLi-mediated dimerization of aryl halides

Scheme 13 Atroposelective total synthesis of mastigophorene A (17) (Minnaard and Feringa, 2016). ${ }^{147}$ 
(17) began with a palladium-catalyzed asymmetric conjugate addition of arylboronic acid 166 to 3-methylcyclopent-2-enone (156) under Minnaard's conditions ${ }^{143}$ to afford adduct 167 in $55 \%$ yield with $92 \%$ ee. Dehydrogenation ${ }^{151}$ of freshly prepared adduct 167 generated enone 169, which was converted to dimethylherbertenediol (170) in three steps. Aryl bromide 171 was obtained by bromination of 170. Homocoupling of the enantiomerically pure mastigophorene building block 171 was achieved with 5 mol\% Pd-PEPPSI-IPent (172). The homocoupling involved conversion of $\mathbf{1 7 1}$ to the corresponding lithium salt via halogen/lithium exchange with tert-butyllithium (1.2 equiv.) to produce a biaryl product with a 9:1 dr (major $P$ helicity of the biaryl axis). Subsequent treatment of the biaryl with $\mathrm{BBr}_{3}$ afforded mastigophorene A (17) in $27 \%$ yield over two steps from 171. The authors reasoned that the observed diastereoselectivity was the result of a catalyst-induced point-<smiles>C=CCCCC[C@]1(C[N+](=O)[O-])CCCC(=O)C1</smiles>

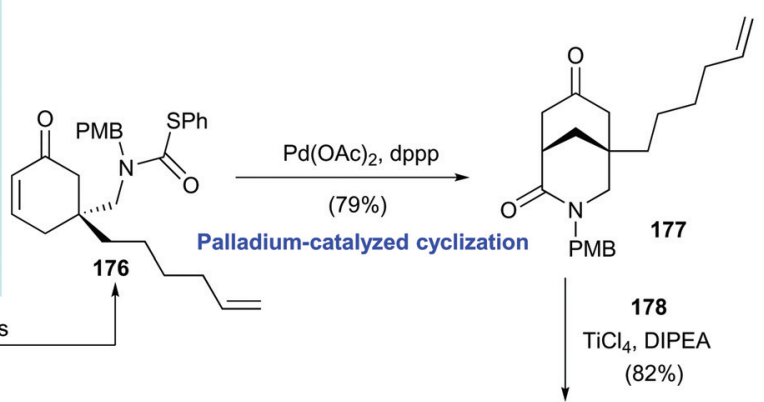

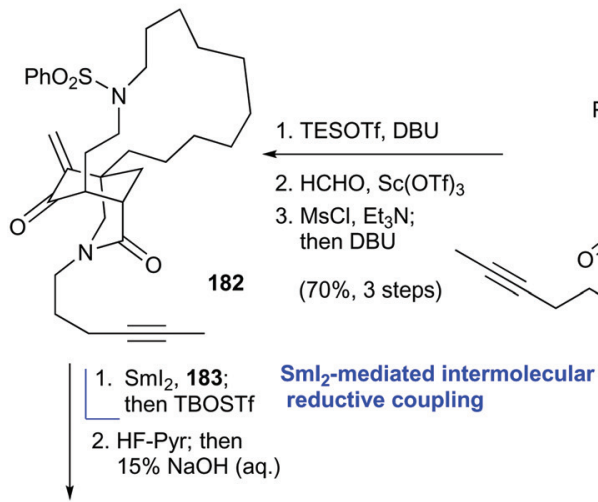

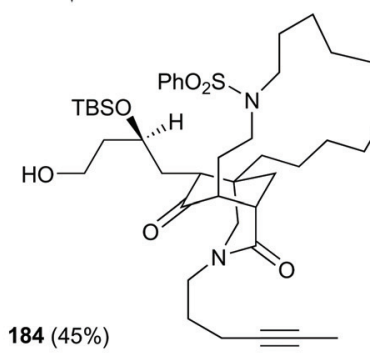

Other isomer $22 \%$
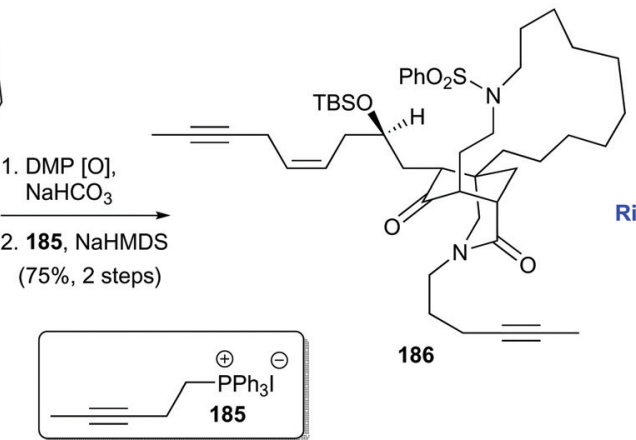
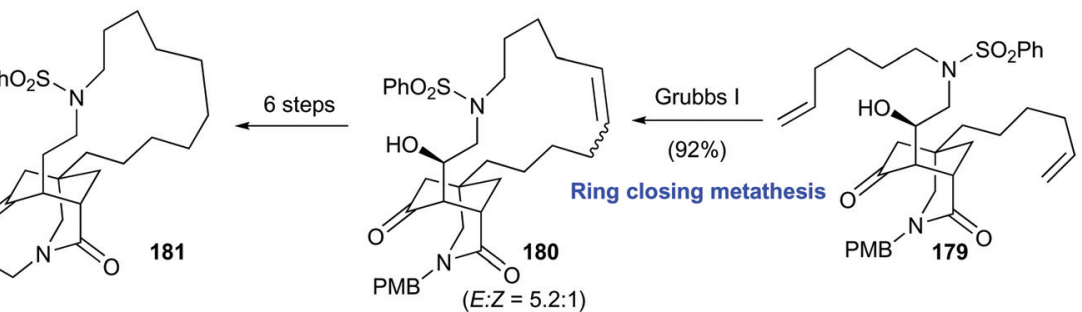

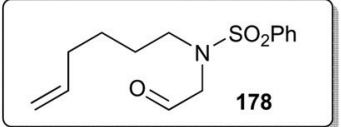


to-axial chirality transfer involving a steric interaction between the aromatic residues of the catalyst (Pd-PEPPSI-Ipent 172) and the benzylic quaternary stereocenter at the para position.

\section{Construction of all-carbon quaternary stereocenters by organocatalytic asymmetric conjugate addition}

The construction of quaternary stereocenters by organocatalytic reactions has long been a research interest in organic chemistry. ${ }^{10,152,153}$ It not only enables the construction of stereocenter(s) without the use of metal catalysts but also is useful in the synthesis of complex natural products. ${ }^{154-157}$ However, only a few examples of all-carbon quaternary stereocenter construction via organocatalytic asymmetric conjugate addition in natural product synthesis have been reported. ${ }^{158}$ In 2016, Huang and co-workers reported an elegant synthesis of (-)-haliclonin A (18) by using a novel organocatalytic asymmetric conjugate addition of nitromethane $e^{159-163}$ to enone 173, with the thiourea catalyst 174 (Scheme 14). ${ }^{164}$

\section{1. (-)-Haliclonin A (Huang 2016) ${ }^{164,165}$}

(-)-Haliclonin A (18) is a macrocyclic alkaloid. It was first isolated from a marine sponge Haliclona sp. by Shin and co-
A.(i) Copper-catalyzed asymmetric conjugate addition of alkyl group

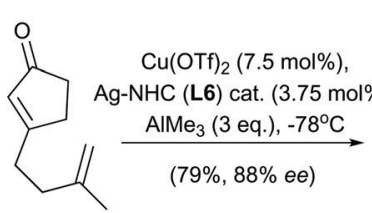

100

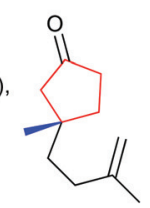

101

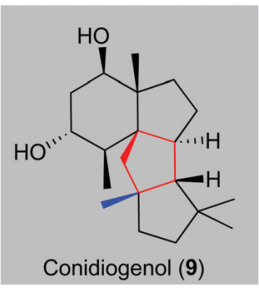

Conidiogenol (9) (ii) Copper-catalyzed asymmetric conjugate addition alkyl group/enolate trapping

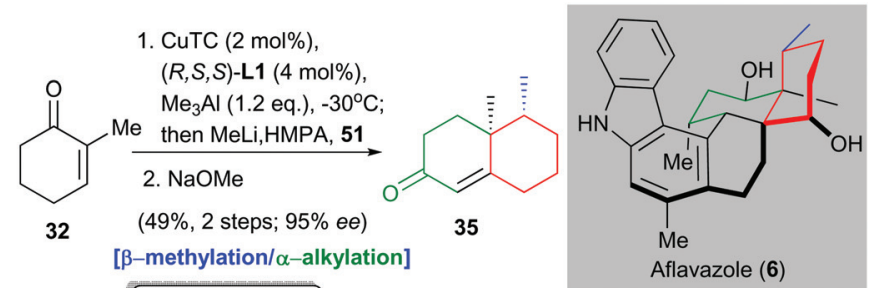

B. Palladium-catalyzed asymmetric conjugate addition of arylboronic acid

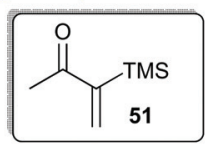

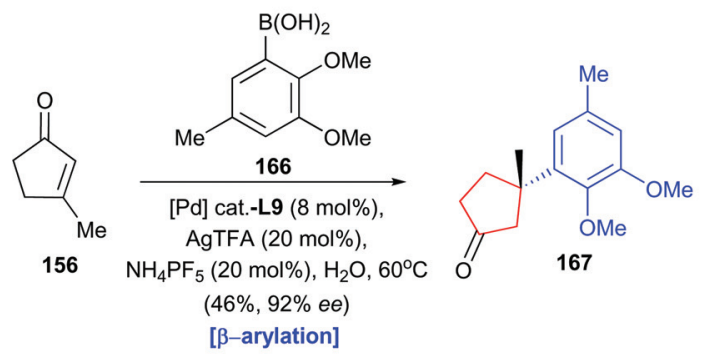

C. Organocatalytic asymmetric conjugate addition of nitromethane<smiles>C=CCCCCC1=CC(=O)CCC1</smiles>

173

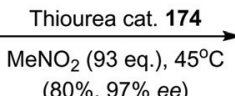
(80\%, 97\% ee) [ $\beta$-nitromethylation]<smiles>N[C@@H]1CCCC[C@H]1NC(=S)Nc1cc(C(F)(F)F)cc(C(F)(F)F)c1</smiles>
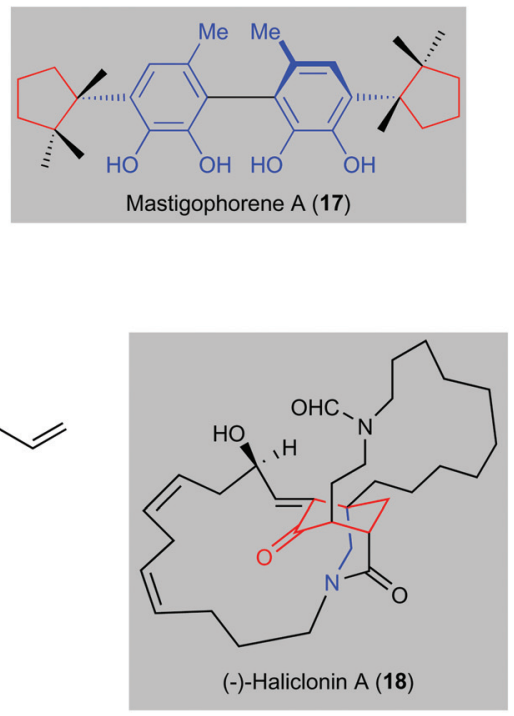

175<smiles>C=CCCCC[C@]1(C[N+](=O)[O-])CCCC(=O)C1</smiles>

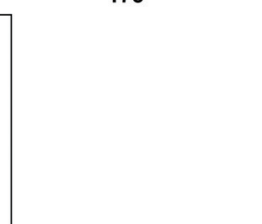

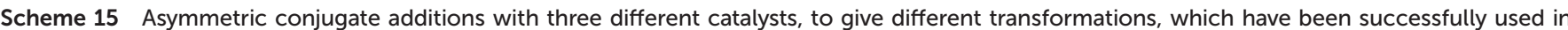

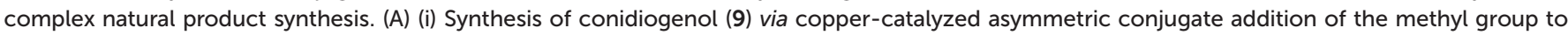

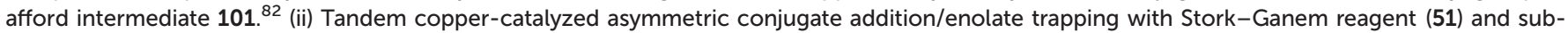

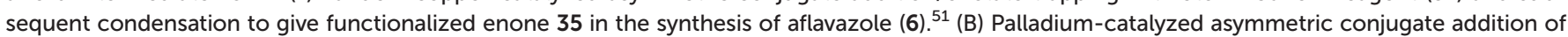

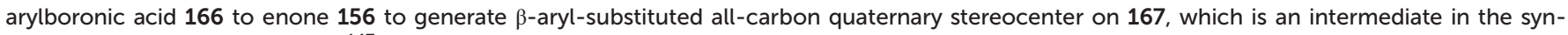

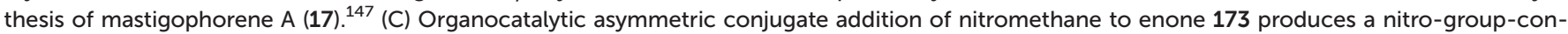
taining adduct 175 , which is used in the total synthesis of (-)-haliclonin A (18). ${ }^{164}$ 
A.<smiles>C=C(C)CCC1(C)CCC(=O)C1</smiles>

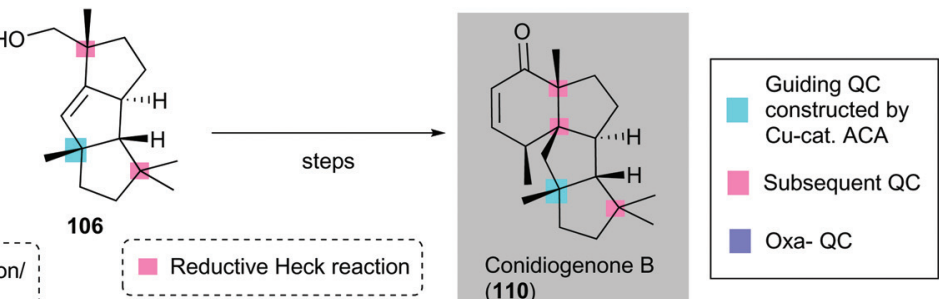

B.

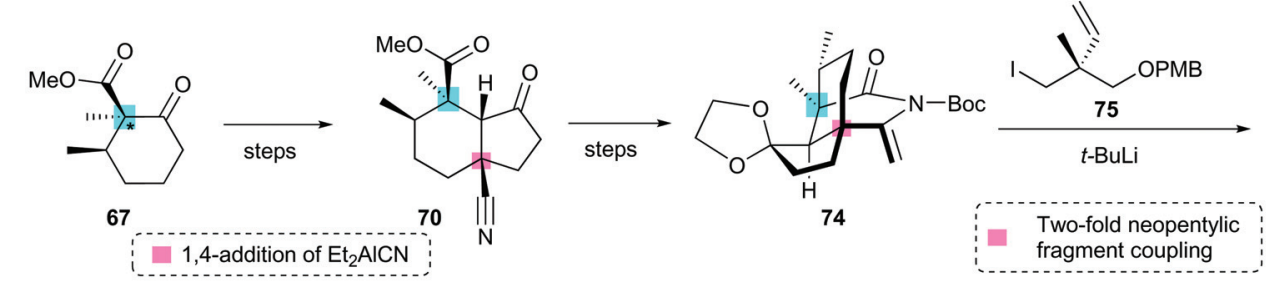

C.<smiles>COc1cccc(C[C@@H](O)[C@H]2C(=O)CCC[C@@]2(C)CO)c1OC</smiles>

87<smiles>C/C=C/[C@@]1(CCc2cccc(OC)c2OC)C(=O)CCC[C@]1(C)C#N</smiles><smiles>C1CCCCC1</smiles>

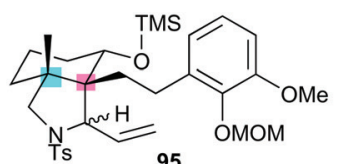
palladium-catalyzed
decarboxylative allylation steps $\mid$\begin{tabular}{c|c} 
\\
$-\ldots$
\end{tabular}

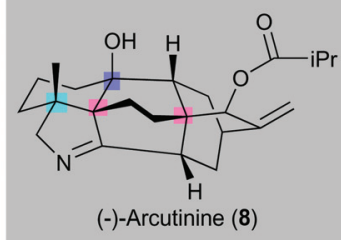
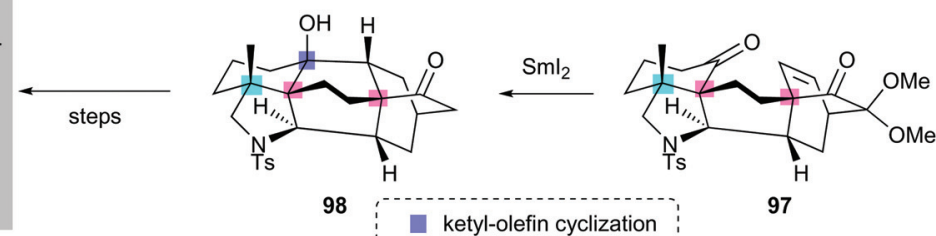

98

ketyl-olefin cyclization
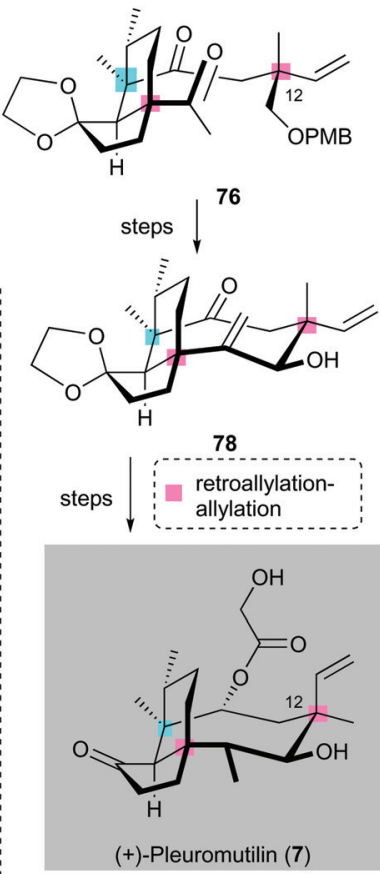

D.<smiles>C=C1C(=O)CCC[C@@]1(C)CCC#CC</smiles>

118

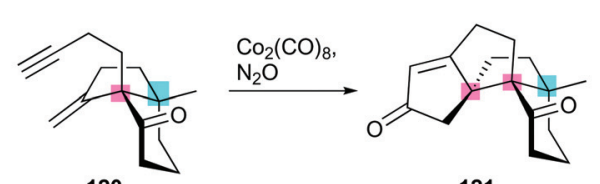

120

121

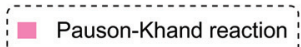

Conia-ene $\quad$ Pauson-Khand reaction cyclization

E.

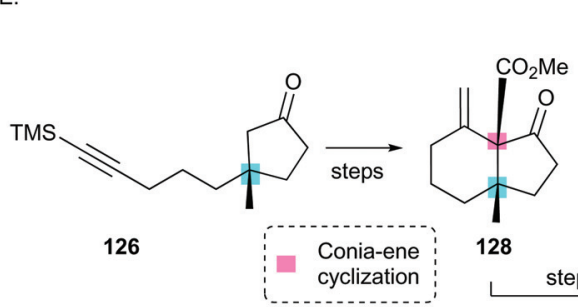

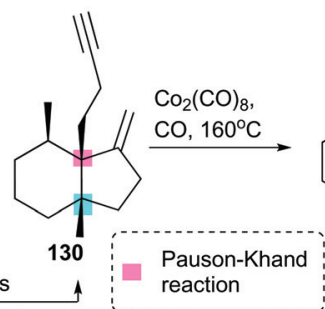

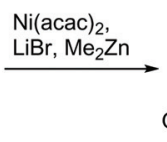

97

(+)-Pleuromutilin (7)

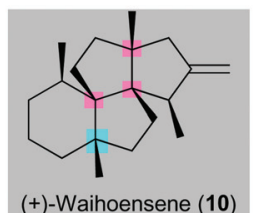

122

(+)-Waihoensene (10)

Scheme 16 Quaternary-center-guided synthetic analysis of natural product synthesis with multiple quaternary centers (QCs) using selected examples in this review. (Guiding QC is highlighted in cyan; subsequent QC is highlighted in pink, and Oxa-QC is highlighted in lilac.) (A) Synthesis of conidiogenone B (110) reported by Snyder [three subsequent QCs]. (B) Synthesis of (+)-pleuromutilin (7) reported by Herzon ${ }^{59}$ [two subsequent QCs]. (*Guiding QC is synthesized through enolate trapping and $\alpha$-methylation after asymmetric conjugate addition.) (C) Synthesis of (-)-arcutinine

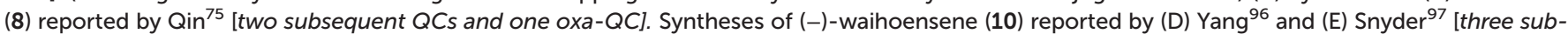
sequent $Q C s$ ]. Cu ACA = copper-catalyzed asymmetric conjugate addition. 
workers in $2009 .{ }^{166}$ It shows moderate antibacterial activity and cytotoxicity against the K562 leukemia cell line. An asymmetric total synthesis of $\mathbf{1 8}$ was reported by Huang and coworkers in 2016. ${ }^{164}$ They used an organocatalytic asymmetric conjugate addition of nitromethane to enone $\mathbf{1 7 3}$ to configure the stereochemistry of the all-carbon quaternary stereocenter (Scheme 14). ${ }^{164,165}$ The synthesis began with asymmetric conjugate addition of nitromethane to enone 173, with chiral thiourea $\mathbf{1 7 4}$ as the catalyst, to give adduct 175 in $80 \%$ yield with $97 \%$ ee. Enone 176, which was formed in six steps from the enantioenriched adduct $\mathbf{1 7 5}$, was subjected to palladiumpromoted cyclization ${ }^{167}$ to form the 3-azabicyclo[3,3,1]nonane core 177 in $79 \%$ yield. $\mathrm{A} \mathrm{TiCl}_{4} / \mathrm{Hünig}$ base-mediated aldol reaction $^{168}$ of 177 with aldehyde $178^{167}$ produced 179 in $82 \%$ yield; 179 underwent ring-closing metathesis with Grubbs I catalyst $^{169}$ to give $\mathbf{1 8 0}$ as a geometric mixture in $92 \%$ yield.

A six-step synthesis from 180 gave alkyne 181, which was subjected to $\alpha$-methylenation via a sequence of reactions, namely silyl enol ether formation with TESOTf/DBU, $\alpha$-hydroxymethylation with formalin/Sc(OTf $)_{3},{ }^{170}$ and mesylation/elimination by DBU. Enone 182 was obtained in 70\% yield over three steps. Intermolecular reductive coupling of freshly prepared 182 with aldehyde 183 was facilitated by samarium diiodide $^{171}$ and the resultant alcohol was subjected to silylation with TBSOTf. Selective desilylation of the primary silyl ether gave the desired diastereomer 184 in $45 \%$ yield and the undesired isomer (not shown) in $22 \%$ yield. Oxidation of newly formed $\mathbf{1 8 4}$ with Dess-Martin periodinane and a subsequent Wittig reaction with $185^{172}$ afforded enediyne 186 in $75 \%$ yield over two steps. Ring-closing alkyne metathesis ${ }^{173,174}$ of enediyne 186 with Fürstner's catalyst $187^{175}$ gave the cyclization product 188 in $70 \%$ yield. Controlled hydrogenation of 188 by using Lindler's catalyst gave the tetracyclic diene (13Z,16Z)-189 in 95\% yield. Desulfonylation of 189 with magnesium in methanol under ultrasonic irradiation ${ }^{176}$ and immediate formylation of the liberated amine with ethyl formate/pyridine afforded 190 in $82 \%$ yield over two steps. Treatment of freshly prepared ketone 190 with TMSI-HMDS ${ }^{177}$ gave the corresponding silyl enol ether, which was subjected to desilylation with AcOH/silica gel to give an enol (not shown, $85 \%$ yield over two steps). The enol could have been formed via intramolecular hydrogen-bond formation with the lactam. ${ }^{178}$ Oxidation of the resultant enol to the corresponding enone was achieved by using an oxoammonium salt $\left(\mathrm{AZADO}^{+} \mathrm{BF}_{4}{ }^{-}\right.$, 191). ${ }^{179,180}$ Subsequent cleavage of the TBS group by using tris(dimethylamino)sulfonium difluorotrimethylsilicate (TASF) ${ }^{181}$ furnished (-)-haliclonin A (18) in $82 \%$ yield.

\section{Summary and outlook}

All-carbon quaternary stereocenter construction poses a distinct challenge in modern synthetic organic chemistry. The overall efficiency of a natural product synthesis is frequently governed by the methods used to install the all-carbon qua- ternary stereocenters. ${ }^{13}$ This issue is not trivial because few effective methods are available for achieving inversion of undesired configurations of all-carbon quaternary stereocenters to the desired ones. In this review, the use of catalytic asymmetric conjugate additions in natural product synthesis was discussed. Such reactions can be used to build all-carbon quaternary stereocenters with high enantioselectivities, and possibly on a preparative scale (i.e., decagram scale). Only catalytic amounts of a catalyst and a chiral ligand are necessary, which makes this an attractive synthetic method.

In this review, we have summarized many recent developments (2016-2020) in the use of catalytic asymmetric conjugate addition in natural product synthesis. The role of this class of reactions in the synthesis of structurally elusive natural products has been highlighted. Asymmetric conjugate additions with different catalysts, i.e., copper catalysts, palladium catalysts, and organocatalysts, give different transformations, which have been successfully used in complex natural product synthesis (Scheme 15). Examples are copper-catalyzed asymmetric conjugate addition of alkyl groups (e.g., Snyder's synthesis of conidiogenol 9), tandem copper-catalyzed asymmetric conjugate addition/enolate trapping (e.g., Li's synthesis of aflavazole 6), palladium-catalyzed asymmetric conjugate addition of arylboronic acids (e.g., Minnaard and Feringa's synthesis of mastigophorene A 17), and organocatalytic asymmetric conjugate addition of nitromethane [e.g., Huang's synthesis of (-)-haliclonin A 18]. These transformations can work complementarily and expand the range of synthetic methods available for constructing all-carbon quaternary stereocenters with diverse functionalities. Recently, Snyder and co-workers completed their enantioselective synthesis of conidiogenones and postulated the concept of quaternary-center-guided synthetic analysis in the synthesis of natural products containing multiple quaternary stereocenters ${ }^{82}$ (Scheme 16). We attempted to apply this logic to show how a quaternary center forged by catalytic asymmetric conjugate addition could guide successive assembly of subsequent quaternary centers and enable enantioselective synthesis of complex natural products, particularly those bearing congested arrays of stereocenters, including all-carbon quaternary stereocenter(s).

Asymmetric conjugate addition catalyzed by a transition metal such as copper or palladium requires the use of a chiral ligand such as a phosphine ligand [e.g., $(S, R, R)-\mathbf{L 1}]$, a silverchiral NHC complex (e.g., Ag-L5), or a chiral NHC ligand (e.g., L4) to achieve stereocontrol. The synthesis of (-)-haliclonin A (18) reported by Huang ${ }^{164}$ shows that construction of an allcarbon quaternary stereocenter can be achieved by using chiral thiourea 174, which acts as an organocatalyst and can be easily prepared by using an economical and optically pure 1,2diamine as the starting material. ${ }^{182}$ This provides an excellent example of how the structure of the chiral thiourea catalyst 174 can be conveniently varied by altering the source of the 1,2diamine. We predict that further investigation of readily available chiral ligands in transition-metal catalysts and/or novel organocatalysts will promote the development of this method and broaden the range of synthetic applications of asymmetric 
conjugate additions. Palladium-catalyzed asymmetric conjugate additions of $\beta$-aryl groups, which make use of various commercially available, air- and/or moisture-stable arylboronic acids bearing a wide range of functionalities such as halo and nitro groups, undoubtedly provide a convenient and useful approach to the construction of enantioenriched $\beta$-aryl ketones $^{135-138,143}$ (see Schemes 10 and 11). Unlike palladiumcatalyzed asymmetric conjugate additions of boronic acids, the copper-catalyzed counterparts usually require air- and/or moisture-sensitive organometallic nucleophiles generated from arylmagnesium, ${ }^{125,183}$ arylaluminum, ${ }^{47,184,185}$ and arylzinc reagents, ${ }^{105,186,187}$ which are incompatible with many reactive functional groups because of their reactivity with the nucleophiles used. Recently, Zhou achieved asymmetric conjugate addition of organoboron reagents to acyclic enones by combining cost-effective copper catalysts and air-stable organoboron reagents. ${ }^{188}$ However, the use of cyclic enones as substrates is yet to be reported. Finally, we anticipate that enantioenriched scaffolds containing all-carbon quaternary stereocenters constructed by catalytic asymmetric conjugate addition and from chiral pools ${ }^{189}$ will cooperatively provide a wide range of enantioenriched substances. This will improve the synthetic efficiency to meet the demands of academia, materials science, and the pharmaceutical industry.

\section{Conflicts of interest}

There are no conflicts to declare.

\section{Acknowledgements}

Financial support from the Shenzhen Human Resources and Social Security Bureau (50820190066) is gratefully acknowledged. The author thanks C. Hui (Max Planck Institute of Molecular Physiology) for helpful discussion during the preparation of this manuscript and language polishing. The author would like to thank the anonymous reviewers for their thought-provoking comments.

\section{Notes and references}

1 B. M. Trost and C. H. Jiang, Catalytic enantioselective construction of all-carbon quaternary stereocenters, Synthesis, 2006, 369-396, DOI: 10.1055/s-2006-926302.

2 B. M. Wang and Y. Q. Tu, Stereoselective Construction of Quaternary Carbon Stereocenters via a Semipinacol Rearrangement Strategy, Acc. Chem. Res., 2011, 44, 12071222.

3 K. W. Quasdorf and L. E. Overman, Catalytic enantioselective synthesis of quaternary carbon stereocentres, Nature, 2014, 516, 181-191.

4 R. Long, J. Huang, J. X. Gong and Z. Yang, Direct construction of vicinal all-carbon quaternary stereocenters in natural product synthesis, Nat. Prod. Rep., 2015, 32, 15841601.

5 X. P. Zeng, Z. Y. Cao, Y. H. Wang, F. Zhou and J. Zhou, Catalytic Enantioselective Desymmetrization Reactions to All-Carbon Quaternary Stereocenters, Chem. Rev., 2016, 116, 7330-7396.

6 J. J. Feng, M. Holmes and M. J. Krische, Acyclic Quaternary Carbon Stereocenters via Enantioselective Transition Metal Catalysis, Chem. Rev., 2017, 117, 1256412580.

7 H. L. Li and Y. X. Lu, Enantioselective Construction of AllCarbon Quaternary Stereogenic Centers by Using Phosphine Catalysis, Asian J. Org. Chem., 2017, 6, 11301145.

8 Y. Li and S. L. Xu, Transition-Metal-Catalyzed C-H Functionalization for Construction of Quaternary Carbon Centers, Chem. - Eur. J., 2018, 24, 16218-16245.

9 P.-W. Xu, J.-S. Yu, C. Chen, Z.-Y. Cao, F. Zhou and J. Zhou, Catalytic Enantioselective Construction of Spiro Quaternary Carbon Stereocenters, ACS Catal., 2019, 9, 1820-1882.

10 D. Pierrot and I. Marek, Synthesis of Enantioenriched Vicinal Tertiary and Quaternary Carbon Stereogenic Centers within an Acyclic Chain, Angew. Chem., Int. Ed., 2020, 59, 36-49.

11 F. Zhou, L. Zhu, B. Pan, Y. Shi, Y.-L. Liu and J. Zhou, Catalytic enantioselective construction of vicinal quaternary carbon stereocenters, Chem. Sci., 2020, 11, 9341-9365.

12 C. Zheng and S.-L. You, Catalytic asymmetric dearomatization (CADA) reaction-enabled total synthesis of indolebased natural products, Nat. Prod. Rep., 2019, 36, 15891605.

13 C. Li, S. S. Ragab, G. Liu and W. Tang, Enantioselective formation of quaternary carbon stereocenters in natural product synthesis: a recent update, Nat. Prod. Rep., 2020, 37, 276-292.

14 C. N. Ungarean, E. H. Southgate and D. Sarlah, Enantioselective polyene cyclizations, Org. Biomol. Chem., 2016, 14, 5454-5467.

15 A. G. M. Barrett, T. K. Ma and T. Mies, Recent Developments in Polyene Cyclizations and Their Applications in Natural Product Synthesis, Synthesis, 2019, 67-82.

16 C. Bolm, J. P. Hildebrand, K. Muñiz and N. Hermanns, Catalyzed Asymmetric Arylation Reactions, Angew. Chem., Int. Ed., 2001, 40, 3284-3308.

17 A. Y. Hong and B. M. Stoltz, The Construction of AllCarbon Quaternary Stereocenters by Use of Pd-Catalyzed Asymmetric Allylic Alkylation Reactions in Total Synthesis, Eur. J. Org. Chem., 2013, 2745-2759.

18 C. X. Zhuo, C. Zheng and S. L. You, Transition-MetalCatalyzed Asymmetric Allylic Dearomatization Reactions, Acc. Chem. Res., 2014, 47, 2558-2573.

19 Y. Y. Liu, S. J. Han, W. B. Liu and B. M. Stoltz, Catalytic Enantioselective Construction of Quaternary Stereocenters: Assembly of Key Building Blocks for the 
Synthesis of Biologically Active Molecules, Acc. Chem. Res., 2015, 48, 740-751.

20 C. Hui, F. Pu and J. Xu, Metal-Catalyzed Asymmetric Michael Addition in Natural Product Synthesis, Chem. Eur. J., 2017, 23, 4023-4036.

21 A. Alexakis and C. Benhaim, Enantioselective copper-catalysed conjugate addition, Eur. J. Org. Chem., 2002, 32213236.

22 J. Christoffers, G. Koripelly, A. Rosiak and M. Rossle, Recent advances in metal-catalyzed asymmetric conjugate additions, Synthesis, 2007, 1279-1300, DOI: 10.1055/ s-2007-966005.

23 A. Alexakis, J. E. Backvall, N. Krause, O. Pamies and M. Dieguez, Enantioselective copper-catalyzed conjugate addition and allylic substitution reactions, Chem. Rev., 2008, 108, 2796-2823.

24 T. Jerphagnon, M. G. Pizzuti, A. J. Minnaard and B. L. Feringa, Recent advances in enantioselective coppercatalyzed 1,4-addition, Chem. Soc. Rev., 2009, 38, 10391075.

25 C. Hawner and A. Alexakis, Metal-catalyzed asymmetric conjugate addition reaction: formation of quaternary stereocenters, Chem. Commun., 2010, 46, 7295-7306.

26 Z. Galestokova and R. Sebesta, Domino Reactions Initiated by Enantioselective Cu-Catalyzed Conjugate Addition, Eur. J. Org. Chem., 2012, 6688-6695, DOI: 10.1002/ejoc.201200875.

27 B. L. Feringa, M. Pineschi, L. A. Arnold, R. Imbos and A. H. M. de Vries, Highly Enantioselective Catalytic Conjugate Addition and Tandem Conjugate AdditionAldol Reactions of Organozinc Reagents, Angew. Chem., Int. Ed. Engl., 1997, 36, 2620-2623.

28 A. Alexakis, C. Benhaim, S. Rosse and M. Humam, Dramatic improvement of the enantiomeric excess in the asymmetric conjugate addition reaction using new experimental conditions, J. Am. Chem. Soc., 2002, 124, 52625263.

29 B. L. Feringa, Phosphoramidites: Marvellous Ligands in Catalytic Asymmetric Conjugate Addition, Acc. Chem. Res., 2000, 33, 346-353.

30 A. Mendoza, Y. Ishihara and P. S. Baran, Scalable enantioselective total synthesis of taxanes, Nat. Chem., 2012, 4, 21-25.

31 J. Y. W. Mak and C. M. Williams, Enantioselective total synthesis of (-)-neovibsanin $\mathrm{G}$ and (-)-14-epi-neovibsanin G, Chem. Commun., 2012, 48, 287-289.

32 T. L. May, J. A. Dabrowski and A. H. Hoveyda, Formation of Vinyl-, Vinylhalide- or Acyl-Substituted Quaternary Carbon Stereogenic Centers through NHC-Cu-Catalyzed Enantioselective Conjugate Additions of Si-Containing Vinylaluminums to $\beta$-Substituted Cyclic Enones, J. Am. Chem. Soc., 2011, 133, 736-739.

33 T. L. May, J. A. Dabrowski and A. H. Hoveyda, Correction to "Formation of Vinyl-, Vinylhalide- or Acyl-Substituted Quaternary Carbon Stereogenic Centers through NHC-CuCatalyzed Enantioselective Conjugate Additions of Si-
Containing Vinylaluminums to $\beta$-Substituted Cyclic Enones", J. Am. Chem. Soc., 2014, 136, 10544-10544.

34 D. Müller, M. Tissot and A. Alexakis, New Experimental Conditions for Tandem hydroalumination/Cu-Catalyzed Asymmetric Conjugate Additions to $\beta$-Substituted Cyclic Enones, Org. Lett., 2011, 13, 3040-3043.

35 F. Gao and A. H. Hoveyda, alpha-Selective Ni-Catalyzed Hydroalumination of Aryl- and Alkyl-Substituted Terminal Alkynes: Practical Syntheses of Internal Vinyl Aluminums, Halides, or Boronates, J. Am. Chem. Soc., 2010, 132, 10961-10963.

36 D. S. Müller, N. L. Untiedt, A. P. Dieskau, G. L. Lackner and L. E. Overman, Constructing Quaternary Stereogenic Centers Using Tertiary Organocuprates and Tertiary Radicals. Total Synthesis of trans-Clerodane Natural Products, J. Am. Chem. Soc., 2015, 137, 660-663.

37 M. Vuagnoux-d'Augustin and A. Alexakis, CopperCatalyzed Asymmetric Conjugate Addition of Trialkylaluminium Reagents to Trisubstituted Enones: Construction of Chiral Quaternary Centers, Chem. - Eur. J., 2007, 13, 9647-9662.

38 Z. H. Lu, H. L. Li, M. Bian and A. Li, Total Synthesis of Epoxyeujindole A, J. Am. Chem. Soc., 2015, 137, 1376413767.

39 L. M. Chapman, J. C. Beck, L. L. Wu and S. E. Reisman, Enantioselective Total Synthesis of (+)-Psiguadial B, J. Am. Chem. Soc., 2016, 138, 9803-9806.

40 M. Shao, Y. Wang, Z. Liu, D.-M. Zhang, H.-H. Cao, R.-W. Jiang, C.-L. Fan, X.-Q. Zhang, H.-R. Chen, X.-S. Yao and W.-C. Ye, Psiguadials A and B, Two Novel Meroterpenoids with Unusual Skeletons from the Leaves of Psidium guajava, Org. Lett., 2010, 12, 50405043.

41 M. d'Augustin, L. Palais and A. Alexakis, Enantioselective Copper-Catalyzed Conjugate Addition to Trisubstituted Cyclohexenones: Construction of Stereogenic Quaternary Centers, Angew. Chem., Int. Ed., 2005, 44, 1376-1378.

42 B. Suchand, J. Krishna, K. Mritunjoy and G. Satyanarayana, Lewis acid promoted $\mathrm{C}-\mathrm{C}$ and coppercatalyzed $\mathrm{C}-\mathrm{O}$ bond formation: synthesis of neoflavans, RSC Adv., 2014, 4, 13941-13945.

43 A. Saito, N. Nakajima, A. Tanaka and M. Ubukata, Synthetic studies of proanthocyanidins. Part 2: Stereoselective gram-scale synthesis of procyanidin-B3, Tetrahedron, 2002, 58, 7829-7837.

44 B. H. Lipshutz, R. S. Wilhelm and J. A. Kozlowski, Conjugate addition reactions of .alpha.,.beta.-unsaturated ketones with higher order, mixed organocuprate reagents, $\mathrm{R}_{2} \mathrm{Cu}(\mathrm{CN}) \mathrm{Li}_{2}$, J. Org. Chem., 1984, 49, 3938-3942.

45 B. H. Lipshutz, D. A. Parker, J. A. Kozlowski and S. L. Nguyen, Effects of Lewis acids on higher order, mixed cuprate couplings, Tetrahedron Lett., 1984, 25, 5959-5962.

46 A. Rieche, H. Gross and E. Höft, Über $\alpha$-Halogenäther, IV. Synthesen aromatischer Aldehyde mit Dichlormethylalkyläthern, Chem. Ber., 1960, 93, 88-94. 
47 T. L. May, M. K. Brown and A. H. Hoveyda, Enantioselective synthesis of all-carbon quaternary stereogenic centers by catalytic asymmetric conjugate additions of alkyl and aryl aluminum reagents to five-, six-, and seven-membered-ring beta-substituted cyclic enones, Angew. Chem., Int. Ed., 2008, 47, 7358-7362.

48 A. Ghosh, U. K. Banerjee and R. V. Venkateswaran, Photolysis of $\alpha$-diazocyclopentanones. Ring contraction to functionalised cyclobutanes and synthesis of junionone, grandisol and planococcyl acetate, Tetrahedron, 1990, 46, 3077-3088.

49 B. L. Hodous and G. C. Fu, Enantioselective Addition of Amines to Ketenes Catalyzed by a Planar-Chiral Derivative of PPY: Possible Intervention of Chiral Brønsted-Acid Catalysis, J. Am. Chem. Soc., 2002, 124, 10006-10007.

50 S. L. Wiskur and G. C. Fu, Catalytic Asymmetric Synthesis of Esters from Ketenes, J. Am. Chem. Soc., 2005, 127, 6176-6177.

51 H. Li, Q. Chen, Z. Lu and A. Li, Total Syntheses of Aflavazole and 14-Hydroxyaflavinine, J. Am. Chem. Soc., 2016, 138, 15555-15558.

52 M. R. TePaske, J. B. Gloer, D. T. Wicklow and P. F. Dowd, Aflavazole: a new antiinsectan carbazole metabolite from the sclerotia of Aspergillus flavus, J. Org. Chem., 1990, 55, 5299-5301.

53 G. Stork and B. Ganem, .alpha.-Silylated vinyl ketones. New class of reagents for the annelation of ketones, J. Am. Chem. Soc., 1973, 95, 6152-6153.

54 K. C. Nicolaou, D. L. F. Gray, T. Montagnon and S. T. Harrison, Oxidation of Silyl Enol Ethers by Using IBX and IBX-N-Oxide Complexes: A Mild and Selective Reaction for the Synthesis of Enones, Angew. Chem., Int. Ed., 2002, 41, 996-1000.

55 K.-S. Lee, A. R. Zhugralin and A. H. Hoveyda, Efficient C-B Bond Formation Promoted by N-Heterocyclic Carbenes: Synthesis of Tertiary and Quaternary B-Substituted Carbons through Metal-Free Catalytic Boron Conjugate Additions to Cyclic and Acyclic $\alpha, \beta$-Unsaturated Carbonyls, J. Am. Chem. Soc., 2009, 131, 7253-7255.

56 K.-S. Lee, A. R. Zhugralin and A. H. Hoveyda, Efficient C-B Bond Formation Promoted by N-Heterocyclic Carbenes: Synthesis of Tertiary and Quaternary B-Substituted Carbons through Metal-Free Catalytic Boron Conjugate Additions to Cyclic and Acyclic $\alpha, \beta$-Unsaturated Carbonyls, J. Am. Chem. Soc., 2010, 132, 12766-12766.

57 M. Okutani and Y. Mori, Synthesis of Alkynes from Vinyl Triflates Using Tetrabutylammonium Fluoride, Chem. Pharm. Bull., 2015, 63, 393-396.

58 P. Yang, M. Yao, J. Li, Y. Li and A. Li, Total Synthesis of Rubriflordilactone B, Angew. Chem., Int. Ed., 2016, 55, 6964-6968.

59 S. K. Murphy, M. Zeng and S. B. Herzon, A modular and enantioselective synthesis of the pleuromutilin antibiotics, Science, 2017, 356, 956-959.

60 M. S. Zeng, S. K. Murphy and S. B. Herzon, Development of a Modular Synthetic Route to (+)-Pleuromutilin, (+)-12-
epi-Mutilins, and Related Structures, J. Am. Chem. Soc., 2017, 139, 16377-16388.

61 C. Davidovich, A. Bashan, T. Auerbach-Nevo, R. D. Yaggie, R. R. Gontarek and A. Yonath, Induced-fit tightens pleuromutilins binding to ribosomes and remote interactions enable their selectivity, Proc. Natl. Acad. Sci. U. S. A., 2007, 104, 4291.

62 O. Goethe, A. Heuer, X. S. Ma, Z. X. Wang and S. B. Herzon, Antibacterial properties and clinical potential of pleuromutilins, Nat. Prod. Rep., 2019, 36, 220-247.

63 E. G. Gibbons, TOTAL SYNTHESIS OF (+/-)-Pleuromutilin, J. Am. Chem. Soc., 1982, 104, 1767-1769.

64 M. D. Helm, M. Da Silva, D. Sucunza, T. K. Findley and D. J. Procter, A Dialdehyde Cyclization Cascade: An Approach to Pleuromutilin, Angew. Chem., Int. Ed., 2009, 48, 9315-9317.

65 N. J. Fazakerley, M. D. Helm and D. J. Procter, Total Synthesis of (+)-Pleuromutilin, Chem. - Eur. J., 2013, 19, 6718-6723.

66 E. P. Farney, S. S. Feng, F. Schäfers and S. E. Reisman, Total Synthesis of (+)-Pleuromutilin, J. Am. Chem. Soc., 2018, 140, 1267-1270.

67 S. K. Murphy, M. Zeng and S. B. Herzon, Stereoselective Multicomponent Reactions Using Zincate Nucleophiles: $\beta$-Dicarbonyl Synthesis and Functionalization, Org. Lett., 2016, 18, 4880-4883.

68 R. A. Bunce and C. R. Harris, Six-membered cyclic .beta.keto esters by tandem conjugate addition-Dieckmann condensation reactions, J. Org. Chem., 1992, 57, 6981-6985.

69 G. T. Crisp, W. J. Scott and J. K. Stille, Palladium-catalyzed carbonylative coupling of vinyl triflates with organostannanes. A total synthesis of (.+-.)-.DELTA.9(12)-capnellene, J. Am. Chem. Soc., 1984, 106, 7500-7506.

70 W. He, X. Sun and A. J. Frontier, Polarizing the Nazarov Cyclization: Efficient Catalysis under Mild Conditions, J. Am. Chem. Soc., 2003, 125, 14278-14279.

71 W. Nagata, M. Yoshioka and T. Terasawa, Hydrocyanation. VII. Stereochemistry of conjugate hydrocyanation of cyclic .alpha.,.beta.-unsaturated ketones, J. Am. Chem. Soc., 1972, 94, 4672-4683.

72 T. Tsunoda, M. Suzuki and R. Noyori, A facile procedure for acetalization under aprotic conditions, Tetrahedron Lett., 1980, 21, 1357-1358.

73 H. Wang, S. Negretti, A. R. Knauff and J. Montgomery, Exo-Selective Reductive Macrocyclization of Ynals, Org. Lett., 2015, 17, 1493-1496.

74 H. Berner, H. Vyplel, G. Schulz and H. Schneider, Chemie der Pleuromutiline, 11. Mitt.: Konfigurationsumkehr der Vinylgruppe am Kohlenstoff 12 durch reversible Retro-EnSpaltung, Monatsh. Chem., 1986, 117, 1073-1080.

75 W. Nie, J. Gong, Z. Chen, J. Liu, D. Tian, H. Song, X.-Y. Liu and Y. Qin, Enantioselective Total Synthesis of (-)-Arcutinine, J. Am. Chem. Soc., 2019, 141, 9712-9718.

76 S. V. McCowen, N. A. Doering and R. Sarpong, Retrosynthetic strategies and their impact on synthesis of 
arcutane natural products, Chem. Sci., 2020, 11, 75387552.

77 S. A. Saidkhodzhaeva, I. A. Bessonova and N. D. Abdullaev, ARCUTININE, A NEW ALKALOID FROM Aconitum arcuatum, Chem. Nat. Compd., 2001, 37, 466469.

78 S. Zhou, K. Xia, X. Leng and A. Li, Asymmetric Total Synthesis of Arcutinidine, Arcutinine, and Arcutine, J. Am. Chem. Soc., 2019, 141, 13718-13723.

79 V. Heguaburu, V. Schapiro and E. Pandolfi, Synthesis of an epoxyquinol analog: efficient methodology for the insertion of side chains into cyclohexenone cores, Tetrahedron Lett., 2010, 51, 6921-6923.

80 K. V. Petrova, J. T. Mohr and B. M. Stoltz, Enantioselective Total Synthesis of (+)-Cassiol, Org. Lett., 2009, 11, 293295.

81 P. B. White, J. N. Jaworski, G. H. Zhu and S. S. Stahl, Diazafluorenone-Promoted Oxidation Catalysis: Insights into the Role of Bidentate Ligands in Pd-Catalyzed Aerobic Aza-Wacker Reactions, ACS Catal., 2016, 6, 33403348.

82 P. Hu, H. M. Chi, K. C. DeBacker, X. Gong, J. H. Keim, I. T. Hsu and S. A. Snyder, Quaternary-centre-guided synthesis of complex polycyclic terpenes, Nature, 2019, 569, 703-707.

83 T. Roncal, S. Cordobés, U. Ugalde, Y. He and O. Sterner, Novel diterpenes with potent conidiation inducing activity, Tetrahedron Lett., 2002, 43, 67996802.

84 L. Du, D. Li, T. Zhu, S. Cai, F. Wang, X. Xiao and Q. Gu, New alkaloids and diterpenes from a deep ocean sediment derived fungus Penicillium sp, Tetrahedron, 2009, 65, 1033-1039.

85 S.-S. Gao, X.-M. Li, Y. Zhang, C.-S. Li and B.-G. Wang, Conidiogenones $\mathrm{H}$ and $\mathrm{I}$, Two New Diterpenes of Cyclopiane Class from a Marine-Derived Endophytic Fungus Penicillium chrysogenum QEN-24S, Chem. Biodiversity, 2011, 8, 1748-1753.

86 S.-H. Hou, Y.-Q. Tu, S.-H. Wang, C.-C. Xi, F.-M. Zhang, S.-H. Wang, Y.-T. Li and L. Liu, Total Syntheses of the Tetracyclic Cyclopiane Diterpenes Conidiogenone, Conidiogenol, and Conidiogenone B, Angew. Chem., Int. Ed., 2016, 55, 4456-4460.

87 M. K. Brown and A. H. Hoveyda, Enantioselective Total Synthesis of Clavirolide C. Applications of Cu-Catalyzed Asymmetric Conjugate Additions and Ru-Catalyzed RingClosing Metathesis, J. Am. Chem. Soc., 2008, 130, 1290412906.

88 J. C. Lo, Y. Yabe and P. S. Baran, A Practical and Catalytic Reductive Olefin Coupling, J. Am. Chem. Soc., 2014, 136, 1304-1307.

89 E. J. Corey and D. Enders, Applications of N,N-dimethylhydrazones to synthesis. Use in efficient, positionally and stereochemically selective $\mathrm{C} \cdot \mathrm{C}$ bond formation; oxidative hydrolysis to carbonyl compounds, Tetrahedron Lett., 1976, 17, 3-6.
90 T. Ohshima, K. Kagechika, M. Adachi, M. Sodeoka and M. Shibasaki, Asymmetric Heck Reaction-Carbanion Capture Process. Catalytic Asymmetric Total Synthesis of (-)-d9(12)-Capnellene, J. Am. Chem. Soc., 1996, 118, 71087116.

91 H. Guo, C.-G. Dong, D.-S. Kim, D. Urabe, J. Wang, J. T. Kim, X. Liu, T. Sasaki and Y. Kishi, Toolbox Approach to the Search for Effective Ligands for Catalytic Asymmetric Cr-Mediated Coupling Reactions, J. Am. Chem. Soc., 2009, 131, 15387-15393.

92 Q. Tian and G. Zhang, Recent Advances in the Asymmetric Nozaki-Hiyama-Kishi Reaction, Synthesis, 2016, 4038-4049.

93 P. S. Baran, T. J. Maimone and J. M. Richter, Total synthesis of marine natural products without using protecting groups, Nature, 2007, 446, 404-408.

94 W. A. Herrmann, C. Brossmer, K. Öfele, C.-P. Reisinger, T. Priermeier, M. Beller and H. Fischer, Palladacycles as Structurally Defined Catalysts for the Heck Olefination of Chloro- and Bromoarenes, Angew. Chem., Int. Ed. Engl., 1995, 34, 1844-1848.

95 A. Rosales, I. Rodríguez-García, J. Muñoz-Bascón, E. Roldan-Molina, N. M. Padial, L. P. Morales, M. GarcíaOcaña and J. E. Oltra, The Nugent Reagent: A Formidable Tool in Contemporary Radical and Organometallic Chemistry, Eur. J. Org. Chem., 2015, 4567-4591.

96 Y. Qu, Z. Wang, Z. Zhang, W. Zhang, J. Huang and Z. Yang, Asymmetric Total Synthesis of (+)-Waihoensene, J. Am. Chem. Soc., 2020, 142, 6511-6515.

97 C. Peng, P. Arya, Z. Zhou and S. A. Snyder, A Concise Total Synthesis of (+)-Waihoensene Guided by Quaternary Center Analysis, Angew. Chem., Int. Ed., 2020, 59, 1352113525.

98 D. B. Clarke, S. F. R. Hinkley and R. T. Weavers, Waihoensene. A new laurenene-related diterpene from Podocarpus totara var waihoensis, Tetrahedron Lett., 1997, 38, 4297-4300.

99 H. Lee, T. Kang and H.-Y. Lee, Total Synthesis of ( \pm -Waihoensene, Angew. Chem., Int. Ed., 2017, 56, 82548257.

100 C. Bleschke, M. Tissot, D. Müller and A. Alexakis, Direct Trapping of Sterically Encumbered Aluminum Enolates, Org. Lett., 2013, 15, 2152-2155.

101 D. Hack, M. Blümel, P. Chauhan, A. R. Philipps and D. Enders, Catalytic Conia-ene and related reactions, Chem. Soc. Rev., 2015, 44, 6059-6093.

102 I. Ojima, M. Tzamarioudaki, Z. Li and R. J. Donovan, Transition Metal-Catalyzed Carbocyclizations in Organic Synthesis, Chem. Rev., 1996, 96, 635-662.

103 J. D. Ricker, V. Mohammadrezaei, T. J. Crippen, A. M. Zell and L. M. Geary, Nitrous Oxide Promoted Pauson-Khand Cycloadditions, Organometallics, 2018, 37, 45564559.

104 C. Petrier, J. De Souza Barbosa, C. Dupuy and J. L. Luche, Ultrasound in organic synthesis. 7. Preparation of organozinc reagents and their nickel-catalyzed reactions with . 
alpha.,.beta.-unsaturated carbonyl compounds, J. Org. Chem., 1985, 50, 5761-5765.

105 M. K. Brown, T. L. May, C. A. Baxter and A. H. Hoveyda, All-Carbon Quaternary Stereogenic Centers by Enantioselective Cu-Catalyzed Conjugate Additions Promoted by a Chiral N-Heterocyclic Carbene, Angew. Chem., Int. Ed., 2007, 46, 1097-1100.

106 G. H. Posner and C. M. Lentz, A directing effect of neighboring aromatic groups on the regiochemistry of formation and stereochemistry of alkylation and bromination of ketone lithium enolates. Evidence for lithiumarene coordination and dramatic effect of copper(I) in controlling stereochemistry and limiting polyalkylation, J. Am. Chem. Soc., 1979, 101, 934-946.

107 A. P. Krapcho, J. F. Weimaster, J. M. Eldridge, E. G. E. Jahngen, A. J. Lovey and W. P. Stephens, Synthetic applications and mechanism studies of the decarbalkoxylations of geminal diesters and related systems effected in dimethyl sulfoxide by water and/or by water with added salts, J. Org. Chem., 1978, 43, 138-147.

108 J. J. Kennedy-Smith, S. T. Staben and F. D. Toste, Gold(I)Catalyzed Conia-Ene Reaction of $\beta$-Ketoesters with Alkynes, J. Am. Chem. Soc., 2004, 126, 4526-4527.

109 Y. Kanda, H. Nakamura, S. Umemiya, R. K. Puthukanoori, V. R. M. Appala, G. K. Gaddamanugu, B. R. Paraselli and P. S. Baran, Two-Phase Synthesis of Taxol®, J. Am. Chem. Soc., 2020, 142(23), 10526-10533.

110 R. A. Holton, H. B. Kim, C. Somoza, F. Liang, R. J. Biediger, P. D. Boatman, M. Shindo, C. C. Smith, S. C. Kim, H. Nadizadeh, Y. Suzuki, C. L. Tao, P. Vu, S. H. Tang, P. S. Zhang, K. K. Murthi, L. N. Gentile and J. H. Liu, FIRST TOTAL SYNTHESIS OF TAXOL .2. COMPLETION OF THE C-RING AND D-RING, J. Am. Chem. Soc., 1994, 116, 1599-1600.

111 R. A. Holton, C. Somoza, H. B. Kim, F. Liang, R. J. Biediger, P. D. Boatman, M. Shindo, C. C. Smith, S. C. Kim, H. Nadizadeh, Y. Suzuki, C. L. Tao, P. Vu, S. H. Tang, P. S. Zhang, K. K. Murthi, L. N. Gentile and J. H. Liu, FIRST TOTAL SYNTHESIS OF TAXOL .1. FUNCTIONALIZATION OF THE B-RING, J. Am. Chem. Soc., 1994, 116, 1597-1598.

112 K. C. Nicolaou, Z. Yang, J. J. Liu, H. Ueno, P. G. Nantermet, R. K. Guy, C. F. Claiborne, J. Renaud, E. A. Couladouros, K. Paulvannan and E. J. Sorensen, TOTAL SYNTHESIS OF TAXOL, Nature, 1994, 367, 630634.

113 J. J. Masters, J. T. Link, L. B. Snyder, W. B. Young and S. J. Danishefsky, A TOTAL SYNTHESIS OF TAXOL, Angew. Chem., Int. Ed. Engl., 1995, 34, 1723-1726.

114 T. Mukaiyama, I. Shiina, H. Iwadare, H. Sakoh, Y. Tani, M. Hasegawa and K. Saitoh, Asymmetric total synthesis of Taxol(R), Proc. Jpn. Acad., Ser. B, 1997, 73, 95-100.

115 P. A. Wender, N. F. Badham, S. P. Conway, P. E. Floreancig, T. E. Glass, J. B. Houze, N. E. Krauss, D. S. Lee, D. G. Marquess, P. L. McGrane, W. Meng, M. G. Natchus, A. J. Shuker, J. C. Sutton and R. E. Taylor,
The pinene path to taxanes .6. A concise stereocontrolled synthesis of taxol, J. Am. Chem. Soc., 1997, 119, 27572758.

116 K. Morihira, R. Hara, S. Kawahara, T. Nishimori, N. Nakamura, H. Kusama and I. Kuwajima, Enantioselective total synthesis of taxol, J. Am. Chem. Soc., 1998, 120, 12980-12981.

117 T. Doi, S. Fuse, S. Miyamoto, K. Nakai, D. Sasuga and T. Takahashi, A formal total synthesis of taxol aided by an automated synthesizer, Chem.-Asian J., 2006, 1, 370-383.

118 K. Fukaya, K. Kodama, Y. Tanaka, H. Yamazaki, T. Sugai, Y. Yamaguchi, A. Watanabe, T. Oishi, T. Sato and N. Chida, Synthesis of Paclitaxel. 2. Construction of the ABCD Ring and Formal Synthesis, Org. Lett., 2015, 17, 2574-2577.

119 K. Fukaya, Y. Tanaka, A. C. Sato, K. Kodama, H. Yamazaki, T. Ishimoto, Y. Nozaki, Y. M. Iwaki, Y. Yuki, K. Umei, T. Sugai, Y. Yamaguchi, A. Watanabe, T. Oishi, T. Sato and N. Chida, Synthesis of Paclitaxel. 1. Synthesis of the ABC Ring of Paclitaxel by $\mathrm{SmI}_{2}$-Mediated Cyclization, Org. Lett., 2015, 17, 2570-2573.

120 S. Hirai, M. Utsugi, M. Iwamoto and M. Nakada, Formal Total Synthesis of (-)-Taxol through Pd-Catalyzed EightMembered Carbocyclic Ring Formation, Chem. - Eur. J., 2015, 21, 355-359.

121 K. Chen and P. S. Baran, Total synthesis of eudesmane terpenes by site-selective C-H oxidations, Nature, 2009, 459, 824-828.

122 Y. Ishihara and P. S. Baran, Two-Phase Terpene Total Synthesis: Historical Perspective and Application to the Taxol (R) Problem, Synlett, 2010, 1733-1745, DOI: 10.1055/s-0030-1258123.

123 C. X. Yuan, Y. H. Jin, N. C. Wilde and P. S. Baran, Short, Enantioselective Total Synthesis of Highly Oxidized Taxanes, Angew. Chem., Int. Ed., 2016, 55, 8280-8284.

124 N. C. Wilde, M. Isomura, A. Mendoza and P. S. Baran, Two-Phase Synthesis of (-)-Taxuyunnanine D, J. Am. Chem. Soc., 2014, 136, 4909-4912.

125 S. Kehrli, D. Martin, D. Rix, M. Mauduit and A. Alexakis, Formation of Quaternary Chiral Centers by N-Heterocyclic Carbene-Cu-Catalyzed Asymmetric Conjugate Addition Reactions with Grignard Reagents on Trisubstituted Cyclic Enones, Chem. - Eur. J., 2010, 16, 9890-9904.

126 A. S. Kende, S. Johnson, P. Sanfilippo, J. C. Hodges and L. N. Jungheim, Synthesis of a taxane triene, J. Am. Chem. Soc., 1986, 108, 3513-3515.

127 T. Horiguchi, M. Nagura, Q. Cheng, T. Oritani and T. Kudo, Chemical oxidation of taxoids with m-CPBA and dimethyl dioxirane: Regioselective epoxidation of taxinine J derivatives, Heterocycles, 2000, 53(12), 2629-2642.

128 W. P. Griffith, S. V. Ley, G. P. Whitcombe and A. D. White, Preparation and use of tetra-n-butylammonium per-ruthenate (TBAP reagent) and tetra-n-propylammonium perruthenate (TPAP reagent) as new catalytic oxidants for alcohols, J. Chem. Soc., Chem. Commun., 1987, 1625-1627, DOI: $10.1039 / \mathrm{C} 39870001625$. 
129 W. F. Maier and P. V. R. Schleyer, Evaluation and prediction of the stability of bridgehead olefins, J. Am. Chem. Soc., 1981, 103, 1891-1900.

130 A. B. McEwen and P. V. R. Schleyer, Hyperstable olefins: further calculational explorations and predictions, J. Am. Chem. Soc., 1986, 108, 3951-3960.

131 Y. S. Angelis, N. S. Hatzakis, I. Smonou and M. Orfanopoulos, Oxidation of benzyl alcohols by dimethyldioxirane. The question of concerted versus stepwise mechanisms probed by kinetic isotope effects, Tetrahedron Lett., 2001, 42, 3753-3756.

132 E. Vedejs and J. Little, Aziridinomitosenes by Anionic Cyclization: Deuterium as a Removable Blocking Group, J. Am. Chem. Soc., 2002, 124, 748-749.

133 W. A. Nugent and T. V. RajanBabu, Transition-metal-centered radicals in organic synthesis. Titanium(III)-induced cyclization of epoxy olefins, J. Am. Chem. Soc., 1988, 110, 8561-8562.

134 I. Ojima, C. M. Sun, M. Zucco, Y. H. Park, O. Duclos and S. Kuduk, A highly efficient route to taxotere by the $\beta$-Lactam Synthon Method, Tetrahedron Lett., 1993, 34, 4149-4152.

135 K. Kikushima, J. C. Holder, M. Gatti and B. M. Stoltz, Palladium-Catalyzed Asymmetric Conjugate Addition of Arylboronic Acids to Five-, Six-, and Seven-Membered $\beta$-Substituted Cyclic Enones: Enantioselective Construction of All-Carbon Quaternary Stereocenters, J. Am. Chem. Soc., 2011, 133, 6902-6905.

136 A. L. Gottumukkala, K. Matcha, M. Lutz, J. G. de Vries and A. J. Minnaard, Palladium-Catalyzed Asymmetric Quaternary Stereocenter Formation, Chem. - Eur. J., 2012, 18, 6907-6914.

137 S. Lin and X. Lu, Cationic Pd(II)/Bipyridine-Catalyzed Conjugate Addition of Arylboronic Acids to $\beta, \beta$-Disubstituted Enones: Construction of Quaternary Carbon Centers, Org. Lett., 2010, 12, 2536-2539.

138 J. C. Holder, L. Zou, A. N. Marziale, P. Liu, Y. Lan, M. Gatti, K. Kikushima, K. N. Houk and B. M. Stoltz, Mechanism and Enantioselectivity in Palladium-Catalyzed Conjugate Addition of Arylboronic Acids to $\beta$-Substituted Cyclic Enones: Insights from Computation and Experiment, J. Am. Chem. Soc., 2013, 135, 14996-15007.

139 L. Q. Li, M. M. Li, D. Chen, H. M. Liu, H. C. Geng, J. Lin and H. B. Qin, Catalytic asymmetric formal total synthesis of (+)-dichroanone and (+)-taiwaniaquinone $\mathrm{H}$, Tetrahedron Lett., 2014, 55, 5960-5962.

140 S. E. Shockley, J. C. Holder and B. M. Stoltz, A Catalytic, Enantioselective Formal Synthesis of (+)-Dichroanone and (+)-Taiwaniaquinone H, Org. Lett., 2014, 16, 6362-6365.

141 S. Tang, Y. Xu, J. He, Y. He, J. Zheng, X. Pan and X. She, Application of a Domino Friedel-Crafts Acylation/ Alkylation Reaction to the Formal Syntheses of $( \pm)$-Taiwaniaquinol B and $( \pm)$-Dichroanone, Org. Lett., 2008, 10, 1855-1858.

142 M. Node, M. Ozeki, L. Planas, M. Nakano, H. Takita, D. Mori, S. Tamatani and T. Kajimoto, Efficient
Asymmetric Synthesis of abeo-Abietane-Type Diterpenoids by Using the Intramolecular Heck Reaction, J. Org. Chem., 2010, 75, 190-196.

143 J. Buter, R. Moezelaar and A. Minnaard, Enantioselective palladium catalyzed conjugate additions of ortho-substituted arylboronic acids to beta,beta-disubstituted cyclic enones: total synthesis of herbertenediol, enokipodin A and enokipodin B, Org. Biomol. Chem., 2014, 12, 58835890.

144 W.-D. Xu, L.-Q. Li, M.-M. Li, H.-C. Geng and H.-B. Qin, Catalytic Asymmetric Formal Total Synthesis of (-)-Triptophenolide and (+)-Triptolide, Nat. Prod. Bioprospect., 2016, 6, 183-186.

145 S. M. Kupchan, W. A. Court, R. G. Dailey, C. J. Gilmore and R. F. Bryan, Tumor inhibitors. LXXIV. Triptolide and tripdiolide, novel antileukemic diterpenoid triepoxides from Tripterygium wilfordii, J. Am. Chem. Soc., 1972, 94, 7194-7195.

146 S. Goncalves, P. Hellier, M. Nicolas, A. Wagner and R. Baati, Diastereoselective formal total synthesis of ( \pm -triptolide via a novel cationic cyclization of 2-alkenyl1,3-dithiolane, Chem. Commun., 2010, 46, 57785780 .

147 J. Buter, D. Heijnen, C. Vila, V. Hornillos, E. Otten, M. Giannerini, A. J. Minnaard and B. L. Feringa, Palladium-Catalyzed, tert-Butyllithium-Mediated Dimerization of Aryl Halides and Its Application in the Atropselective Total Synthesis of Mastigophorene A, Angew. Chem., Int. Ed., 2016, 55, 3620-3624.

148 Y. Fukuyama, M. Toyota and Y. Asakawa, Mastigophorenes: novel dimeric isocuparane-type sesquiterpenoids from the liverwort Mastigophora diclados, J. Chem. Soc., Chem. Commun., 1988, 1341-1342, DOI: 10.1039/C39880001341.

149 Y. Fukuyama and Y. Asakawa, Novel neurotrophic isocuparane-type sesquiterpene dimers, mastigophorenes A, B, $\mathrm{C}$ and $\mathrm{D}$, isolated from the liverwort Mastigophora diclados, J. Chem. Soc., Perkin Trans. 1, 1991, 2737-2741, DOI: 10.1039/P19910002737.

150 Y. Fukuyama, K. Matsumoto, Y. Tonoi, R. Yokoyama, H. Takahashi, H. Minami, H. Okazaki and Y. Mitsumoto, Total syntheses of neuroprotective mastigophorenes A and B, Tetrahedron, 2001, 57, 7127-7135.

151 T. Diao, T. J. Wadzinski and S. S. Stahl, Direct aerobic $\alpha, \beta$-dehydrogenation of aldehydes and ketones with a Pd (TFA)2/4,5-diazafluorenone catalyst, Chem. Sci., 2012, 3, 887-891.

152 M. Bella and T. Gasperi, Organocatalytic Formation of Quaternary Stereocenters, Synthesis, 2009, 1583-1614, DOI: $10.1055 / \mathrm{s}-0029-1216796$.

153 F. Vetica, R. M. de Figueiredo, M. Orsini, D. Tofani and T. Gasperi, Recent Advances in Organocatalytic Cascade Reactions toward the Formation of Quaternary Stereocenters, Synthesis, 2015, 2139-2184.

154 O. Andrey, A. Vidonne and A. Alexakis, Organocatalytic Michael addition, a convenient tool in total synthesis. 
First asymmetric synthesis of (-)-botryodiplodin, Tetrahedron Lett., 2003, 44, 7901-7904.

155 M. Reiter, S. Torssell, S. Lee and D. W. C. MacMillan, The organocatalytic three-step total synthesis of $(+)$ - frondosin B, Chem. Sci., 2010, 1, 37-42.

156 X. F. Sun and D. W. Ma, Organocatalytic Approach for the Syntheses of Corynantheidol, Dihydrocorynantheol, Protoemetinol, Protoemetine, and Mitragynine, Chem.Asian J., 2011, 6, 2157-2164.

157 G. Varro, L. Hegedus, A. Simon, A. Balogh, A. Grun, I. Leveles, B. G. Vertessy and I. Kadas, The First Enantioselective Total Synthesis of 0-transDihydronarciclasine, J. Nat. Prod., 2017, 80, 1909-1917.

158 R. R. Liu and J. L. Zhang, Organocatalytic Michael Addition of Indoles to Isatylidene-3-acetaldehydes: Application to the Formal Total Synthesis of (-)-Chimonanthine, Org. Lett., 2013, 15, 2266-2269.

159 C. E. T. Mitchell, S. E. Brenner, J. García-Fortanet and S. V. Ley, An efficient, asymmetric organocatalystmediated conjugate addition of nitroalkanes to unsaturated cyclic and acyclic ketones, Org. Biomol. Chem., 2006, 4, 2039-2049.

160 C. E. T. Mitchell, S. E. Brenner and S. V. Ley, A versatile organocatalyst for the asymmetric conjugate addition of nitroalkanes to enones, Chem. Commun., 2005, 53465348, DOI: 10.1039/B511441A.

161 P. Li, Y. Wang, X. Liang and J. Ye, Asymmetric multifunctional organocatalytic Michael addition of nitroalkanes to $\alpha, \beta$-unsaturated ketones, Chem. Commun., 2008, 33023304, DOI: 10.1039/B804540B.

162 P. Kwiatkowski, K. Dudziński and D. Łyżwa, Effect of High Pressure on the Organocatalytic Asymmetric Michael Reaction: Highly Enantioselective Synthesis of $\gamma$-Nitroketones with Quaternary Stereogenic Centers, Org. Lett., 2011, 13, 3624-3627.

163 X. Gu, Y. Dai, T. Guo, A. Franchino, D. J. Dixon and J. Ye, A General, Scalable, Organocatalytic Nitro-Michael Addition to Enones: Enantioselective Access to All-Carbon Quaternary Stereocenters, Org. Lett., 2015, 17, 15051508.

164 L.-D. Guo, X.-Z. Huang, S.-P. Luo, W.-S. Cao, Y.-P. Ruan, J.-L. Ye and P.-Q. Huang, Organocatalytic, Asymmetric Total Synthesis of (-)-Haliclonin A, Angew. Chem., Int. Ed., 2016, 55, 4064-4068.

165 S.-P. Luo, X.-Z. Huang, L.-D. Guo and P.-Q. Huang, Catalytic Asymmetric Total Synthesis of Macrocyclic Marine Natural Product (-)-Haliclonin A, Chin. J. Chem., 2020, 38, DOI: 10.1002/cjoc.202000291.

166 K. H. Jang, G. W. Kang, J.-E. Jeon, C. Lim, H.-S. Lee, C. J. Sim, K.-B. Oh and J. Shin, Haliclonin A, a New Macrocyclic Diamide from the Sponge Haliclona sp, Org. Lett., 2009, 11, 1713-1716.

167 S.-P. Luo, L.-D. Guo, L.-H. Gao, S. Li and P.-Q. Huang, Toward the Total Synthesis of Haliclonin A: Construction of a Tricyclic Substructure, Chem. - Eur. J., 2013, 19, 8791.
168 D. A. Evans, D. L. Rieger, M. T. Bilodeau and F. Urpi, Stereoselective aldol reactions of chlorotitanium enolates. An efficient method for the assemblage of polypropionaterelated synthons, J. Am. Chem. Soc., 1991, 113, 1047-1049.

169 A. K. Chatterjee, T.-L. Choi, D. P. Sanders and R. H. Grubbs, A General Model for Selectivity in Olefin Cross Metathesis, J. Am. Chem. Soc., 2003, 125, 1136011370.

170 M. Bian, Z. Wang, X. Xiong, Y. Sun, C. Matera, K. C. Nicolaou and A. Li, Total Syntheses of Anominine and Tubingensin A, J. Am. Chem. Soc., 2012, 134, 80788081.

171 P. Girard, J. L. Namy and H. B. Kagan, Divalent lanthanide derivatives in organic synthesis. 1. Mild preparation of samarium iodide and ytterbium iodide and their use as reducing or coupling agents, J. Am. Chem. Soc., 1980, 102, 2693-2698.

172 V. Hickmann, A. Kondoh, B. Gabor, M. Alcarazo and A. Fürstner, Catalysis-Based and Protecting-Group-Free Total Syntheses of the Marine Oxylipins Hybridalactone and the Ecklonialactones A, B, and C, J. Am. Chem. Soc., 2011, 133, 13471-13480.

173 A. Fürstner and G. Seidel, Ring-Closing Metathesis of Functionalized Acetylene Derivatives: A New Entry into Cycloalkynes, Angew. Chem., Int. Ed., 1998, 37, 1734-1736.

174 C. M. Neuhaus, M. Liniger, M. Stieger and K.-H. Altmann, Total Synthesis of the Tubulin Inhibitor WF-1360F Based on Macrocycle Formation through Ring-Closing Alkyne Metathesis, Angew. Chem., Int. Ed., 2013, 52, 5866-5870.

175 J. Heppekausen, R. Stade, R. Goddard and A. Fürstner, Practical New Silyloxy-Based Alkyne Metathesis Catalysts with Optimized Activity and Selectivity Profiles, J. Am. Chem. Soc., 2010, 132, 11045-11057.

176 B. Nyasse, L. Grehn and U. Ragnarsson, Mild, efficient cleavage of arenesulfonamides by magnesium reduction, Chem. Commun., 1997, 1017-1018, DOI: 10.1039/ A701408B.

177 R. D. Miller and D. R. McKean, The Facile Silylation of Aldehydes and Ketones using Trimethylsilyl Iodide: An Exceptionally Simple Procedure for the Generation of Thermodynamically Equilibrated Trimethylsilylenol Ethers, Synthesis, 1979, 730-732.

178 M. Hayashi, M. Shibuya and Y. Iwabuchi, Oxidative Conversion of Silyl Enol Ethers to $\alpha, \beta$-Unsaturated Ketones Employing Oxoammonium Salts, Org. Lett., 2012, 14, 154-157.

179 R. Hara, T. Furukawa, H. Kashima, H. Kusama, Y. Horiguchi and I. Kuwajima, Enantioselective Total Synthesis of (+)-Taxusin, J. Am. Chem. Soc., 1999, 121, 3072-3082.

180 H. Kusama, R. Hara, S. Kawahara, T. Nishimori, H. Kashima, N. Nakamura, K. Morihira and I. Kuwajima, Enantioselective Total Synthesis of (-)-Taxol, J. Am. Chem. Soc., 2000, 122, 3811-3820.

181 K. A. Scheidt, H. Chen, B. C. Follows, S. R. Chemler, D. S. Coffey and W. R. Roush, Tris(dimethylamino)sulfo- 
nium Difluorotrimethylsilicate, a Mild Reagent for the Removal of Silicon Protecting Groups, J. Org. Chem., 1998, 63, 6436-6437.

182 K. Mei, S. Zhang, S. He, P. Li, M. Jin, F. Xue, G. Luo, H. Zhang, L. Song, W. Duan and W. Wang, (S)-Pyrrolidine sulfonamide catalyzed asymmetric direct aldol reactions of aryl methyl ketones with aryl aldehydes, Tetrahedron Lett., 2008, 49, 2681-2684.

183 D. Martin, S. Kehrli, M. d'Augustin, H. Clavier, M. Mauduit and A. Alexakis, Copper-Catalyzed Asymmetric Conjugate Addition of Grignard Reagents to Trisubstituted Enones. Construction of All-Carbon Quaternary Chiral Centers, J. Am. Chem. Soc., 2006, 128, 8416-8417.

184 C. Hawner, K. Li, V. Cirriez and A. Alexakis, CopperCatalyzed Asymmetric Conjugate Addition of Aryl Aluminum Reagents to Trisubstituted Enones: Construction of Aryl-Substituted Quaternary Centers, Angew. Chem., Int. Ed., 2008, 47, 8211-8214.

185 J. A. Dabrowski, M. T. Villaume and A. H. Hoveyda, Enantioselective Synthesis of Quaternary Carbon Stereogenic Centers through Copper-Catalyzed Conjugate
Additions of Aryl- and Alkylaluminum Reagents to Acyclic Trisubstituted Enones, Angew. Chem., Int. Ed., 2013, 52, 8156-8159.

186 K.-S. Lee, M. K. Brown, A. W. Hird and A. H. Hoveyda, A Practical Method for Enantioselective Synthesis of AllCarbon Quaternary Stereogenic Centers through NHC-CuCatalyzed Conjugate Additions of Alkyl- and Arylzinc Reagents to $\beta$-Substituted Cyclic Enones, J. Am. Chem. Soc., 2006, 128, 7182-7184.

187 D. Peña, F. López, S. R. Harutyunyan, A. J. Minnaard and B. L. Feringa, Highly enantioselective Cu-catalysed asymmetric 1,4-addition of diphenylzinc to cyclohexenone, Chem. Commun., 2004, 1836-1837, DOI: 10.1039/ B402868F.

188 C. Wu, G. Yue, C. D.-T. Nielsen, K. Xu, H. Hirao and J. Zhou, Asymmetric Conjugate Addition of Organoboron Reagents to Common Enones Using Copper Catalysts, J. Am. Chem. Soc., 2016, 138, 742-745.

189 Z. G. Brill, M. L. Condakes, C. P. Ting and T. J. Maimone, Navigating the Chiral Pool in the Total Synthesis of Complex Terpene Natural Products, Chem. Rev., 2017, 117, 11753-11795. 